\title{
Neue Funde aus der eisenzeitlichen Hügelnekropole von Vergina, Griechisch Makedonien
}

\author{
von Katerina Rhomiopoulou, Athen, und Imma Kilian-Dirlmeier, Bonn
}

In diesem Artikel stellen wir dic frübeisenzeitlichen Funde aus vier Grabbügeln der Nekropole von Vergina vor, die im Herbst 1970 durch cine Rettungsgrabung untersucht wurden. Obwobl bisher nur ein Teil der Nekropole freigelegt werden konnte (insgesamt 107 der etwa $300 \mathrm{Hügel}$ ), stellen die bis heute zum Vorschein gekommenen Beigaben aus den Gräbern den wichtigsten Fundkomplex der makedonischen Frübeisenzeit dar. Die Publikation der neuen Funde soll cin ergänzender Bcitrag zum bereits Bekannten (Typen der Beigaben, Bestattungssitten) und dessen Interpretation scin. Ein neuer und bemerkenswerter Befund ist die Doppelbestattung im Grab I des Hügels $\Gamma$ (Malamas): Es handelt sich um zwci erwachsene Individuen in paralleler Lage, das eine mit Schwert und Lanze ausgestattet, das andere mit reichem Schmuck, also Mann und Frau. Mit diesem Grab ist in Vergina erstmals die Sitte der Doppelbestattung, d.h. der gleichzeitigen Grablege zweier Personen im selben Grab, nachgewiesen. Doppelbestattungen sind auch im übrigen Griechenland während der protogeometrischen und geometrischen Zeit sehr selten. Aus der besonderen Behandlung dieser Toten und ibrer ungewöhnlichen Beigabenausstattung ergeben sich Hinweise auf die soziale Stellung privilegierter Frauen. Außerdem wurde versucht, durch die Fundlage von Beigaben im Grab zu Aussagen über ibre Tragweise bzw. Funktion zu gelangen.

Cet article présente des matériaux du début de l'Age du Fer de quatre tertre funéraires dans la nécropole de Vergina, qui furent mis au jour en automne 1970 lors de fouilles de sauvetage. Les inventaires connus jusqu'à aujourd'bui, provenantes des tombes, représent le plus important complexe des trouvailles du début de l'Age du Fer macédonien, bien que seule une partie de la nécropole fut étudié (en tout 107 tumuli sur 300). La publication des tombes nouvelles porte un supplément aux résultats déjà obtenus (types d'objets des inventaires, mode de sépulture) et leur interprétation. A remarquer, la double inhumation, dans la tombe I du tumulus $\Gamma$ (Malamas): Il s'agit de deux individus adultes allongés à côte, l'un muni d'une épée et d'une lance, l'autre paré de riche bijoux, sans aucun doute homme et femme. Le rite de l'inhumation double est attesté pour la première fois avec cette tombe à Vergina, c'est à dire l'enterrement simultané de deux personnes dans la même tombe. Les inbumations doubles sont très rares dans le reste de la Grèce pendant l'époque protogéométrique et géométrique. On obtient quelques indices concernant le statut social des femmes privilegiée, en considérant le traitement particulier reservé aux morts ainsi que l'inventaire extraordinaire trouvé dans la tombe. En outre on essaya, en partant de la situation de l'inventaire de tirer quelques conclusions concernant la. fonction des objets et leur position sur le costume.

This paper deals with material from the excavation (Oct-Nov 1970) of four tumuli in the extensive Iron Age cemetery of Vergina. Although only part of the cemetery (there are ca. 300 tumuli) bas been excavated (107 tumuli), the grave goods constitute the most important group of finds of the Early Iron Age in Macedonia. The present article helps to improve our knowledge and interpretation of the finds already discussed in earlier publications (types of objects, customs of inbumation). The most important new evidence derives from the double burial (I) in the tumulus $\Gamma$ (Malamas). It contained two adults buried at the same time and laid side by side in the same grave: a woman with rich adornment, to the left of a man with a sword and a spear. This is the first confirmed example of a double burial from the cemetery of Vergina. Moreover, double burials are known to have been rare in the protogeometric and geometric periods in Greece. This particular feature of the contemporary burial of the two deceased individuals provides new evidence for the status of certain women in the period under discussion. The type of grave offerings, and the position in which they were found, make it possible to offer a new interpretation of their function, or at least to advance new bypotheses.

Das kleine Dorf Vergina liegt am südwestlichen Hügelrand der Ebene von Thessaloniki. Weltweit bekannt wurde es scit 1977, als M. Andronikos in der Großen Toumba ein nicht geplündertes Makedonisches Grab 
entdeckte1. Andronikos war es aber auch, der in den Jahren 1951-1961 zum erstenmal in der seit 1855 bekannten, ausgedehnten eisenzeitlichen Hügelnekropole von Vergina systematische Grabungen durchführte². Durch den Straßenbau bedrohte Grabhügel konnte $\mathrm{Ph}$. Petsas in zwei Rettungsgrabungen 1961-1962 untersuchen ${ }^{3}$. Obwohl mit den 107 gegrabenen Hügeln nur ein Bruchteil der gesamten Nekropole erforscht ist, bilden die Beigaben aus diesen Gräbern einen der bedeutendsten Fundkomplexe aus der frühen Eisenzeit Makedoniens.

Die Untersuchung von vier weiteren Grabhügeln dieser Nekropole, die der griechische Antikendienst 1970 durchführen mußte, ergab einige Ergänzungen zum bis= her Bekanntent. Einer dieser Hügel lag im Feld des A. Lazaridis (Parzelle Nr.631), wo Andronikos bereits den Hügel AZ gegraben hatte. Die drei anderen Grabhügel befanden sich im Feld des Malamas (Parzellen Nr. 607 und 608), also nur wenig westlich der Hügel $\Sigma, Y$

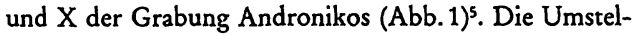
lung von Getreideanbau auf Mandelplantagen war der Anlaß zu einer Rettungsgrabung, die unter schwierigen Witterungsverhältnissen im Oktober und November des Jahres 1970 stattfand. Da diese Hügel durch den Ackerbau bereits stark verschliffen waren, zeichneten sie sich im Gelände nur noch als schwache Erhebungen ab; ihre ursprüngliche Höhe und ihr Umfang waren nicht mehr festzustellen. Selbst die ortsfremde, zur Aufschüttung

1 M. Andronikos, Athens Ann. Arch. 9, 1976, 123 ff.; 10, 1977, $1 \mathrm{ff} . ; 13,1980$, $156 \mathrm{ff} . ;$ ders., Vergina/Oi vasilikoi taphoi (1984) mit weiterer Bibliographie.

2 Andronikos 1969. Die Hügel der Grabung Andronikos sind mit Buchstaben des griechischen Alphabets bezeichnet, die Gräber mit römischen Zahlen. Zur Erleichterung der Übersicht sind im folgenden alle Bezeichnungen von Gräbern bzw. Fundstellen innerhalb Verginer Hügel kursiv gesetzt.

3 Petsas 1961-62; Petsas 1963. Die Hügel der Grabung Pets as sind mit römischen Zahlen bezeichnet, die Gräber mit Buchstaben des griechischen Alphabets (hier kursiv gesetzt, vgl. Anm. 2). Einen zusammenfassenden Bericht über die Publikationen von Andronikos und Petsas gibt W. Radt in: H.Müller-Karpe (Hrsg.), Beiträge zu italienischen und griechischen Bronzefunden. PBF XX,1 (1974) $98 \mathrm{ff}$. Weitere Bemerkungen zur relativen und absoluten Chronologie: Kilian $1975 \mathrm{a}, 65 \mathrm{ff}$.

- Grabung der Ephorie Veria unter Leitung von K. Rhomiopoulou; die Pläne der Hügel werden G.Touratsoglou verdankt. Die im Museum Veria aufbewahrten Funde konnten im Herbst 1986 von den Verf. aufgenommen werden; die Umzeichnungen der Keramik hat Th. Kakarounga besorgt, die der Kleinfunde G. Endlich. Für ihre Hilfe bei der Materialaufnahme im Museum sei X. Tzanavari, V. Alamani und P. Papadopoulos gedankt.

5 Siehe den topographischen Plan mit Flurparzellen: Andronikos 1969, Taf.2. Das Fehlen von Angaben zur Lage des von Petsas untersuchten Nekropolenabschnitts machen eine Lokalisierung im Gelände und eine Verbindung mit dem Gräberplan von Andronikos unmöglich. Die Hügel im Feld Malamas liegen im Nordwesten des westlichsten Hügels (LXXIII) der Grabung Petsas.

- Vgl. Andronikos 1969, 3; 150 f.; Petsas 1963, 227. der Hügel herbeigeschaffte rote Erde $^{6}$ hatte sich in der Ackerkrume so weitgehend mit der hellen, graubraunen Erde der Felder vermischt, daß sie oberflächlich nicht als Verfärbung zu erkennen waren. Die übliche Grabform sind schlichte, ziemlich flache Gruben, in wenigen Ausnahmen mit einer Steinsetzung (z. B. Malamas Hügel A); daneben gab es drei Pithosgräber. Orientierung und Anordnung der Gräber im Hügel entsprechen dem Befund der Grabung Andronikos?.

\section{Der Grabhügel im Feld Lazaridis (Parzelle Nr. 631) (Abb. 2)}

Bei Beginn der Grabung hatte dieser Hügel eine Höhe von nur noch $0,50 \mathrm{~m}$. Die Hügelschüttung bestand aus ortsfremder, reiner roter Erde, die einige wenige Steine enthielt, so daß sich der etwas unregelmäßige Hügelumfang (Durchmesser $11-12,50 \mathrm{~m}$ ) deutlich erkennen ließ.

$\mathrm{Da}$ Andronikos bei dem im gleichen Feld liegenden Hügel AZ zu Beginn der Ausgrabung 1961 keine Störungen der Oberfläche feststellte, muß die Zerstörung des 1970 gegrabenen Hügels durch die Feldbestellung der letzten zehn Jahre verursacht worden sein. In einer Tiefe von $0,35-0,40 \mathrm{~m}$ unter der modernen Oberfläche begannen sich die Gräber abzuzeichnen, meist nicht durch Verfärbungen, sondern allein durch die unterschiedliche Konsistenz der Grubenfüllung; die Grenzen der Grabgruben konnten deshalb in ihrem Verlauf nicht immer exakt festgestellt werden. Insgesamt enthielt dieser Hügel sieben Gräber, nämlich sechs flache Grubengräber und ein Pithosgrab.

Grab I: Flache, rechteckige Grube von ca. $3 \mathrm{~m}$ Länge und ca. 1,40 m Breite; in der Mitte der Grube ein Schwert (c) mit dem Griff zum Krug (a), neben der Schwertspitze Reste eines Langknochens (Oberschenkel?). Der Krug (a) stand wahrscheinlich neben dem Kopf des Toten, der Kantharos (b) bei seinen Füßen (Abb. 3; 4,1-2; 6,1-3; 40,b.e; 41,a-b; 43,b). Der Kantharos (b) aus diesem Grab gehört zu der in Vergina seltenen Gattung der gerieften Keramik ${ }^{8}$. Die Ausführung seiner Riefen findet unter den sieben Gefäßen dieser Gattung aus der Grabung Andronikos keine Entsprechung. Ein chronologisches Merkmal scheint sie jedoch nicht zu sein', da das Eisenschwert (c) dieses Grabes für eine Datierung in die Stufe III A der Nekropole spricht. Den Krug (a) wird man der Gruppe 3 nach Andronikos zuordnen ${ }^{10}$. Auch im Grab AI XI fand sich ein geriefter Kantharos zusammen mit einem Schwert ${ }^{11}$. Eine für Männergräber kenn-

\footnotetext{
7 Andronikos 1969, $151 \mathrm{f}$.

8 Ebd. $185 \mathrm{ff}$.

- Die geriefte Keramik tritt in den Stufen II und III A der Nekropole auf: Kilian 1975 a, $66 \mathrm{f}$.

10 Andronikos 1969, 196.

11 Ebd. 64.
} 


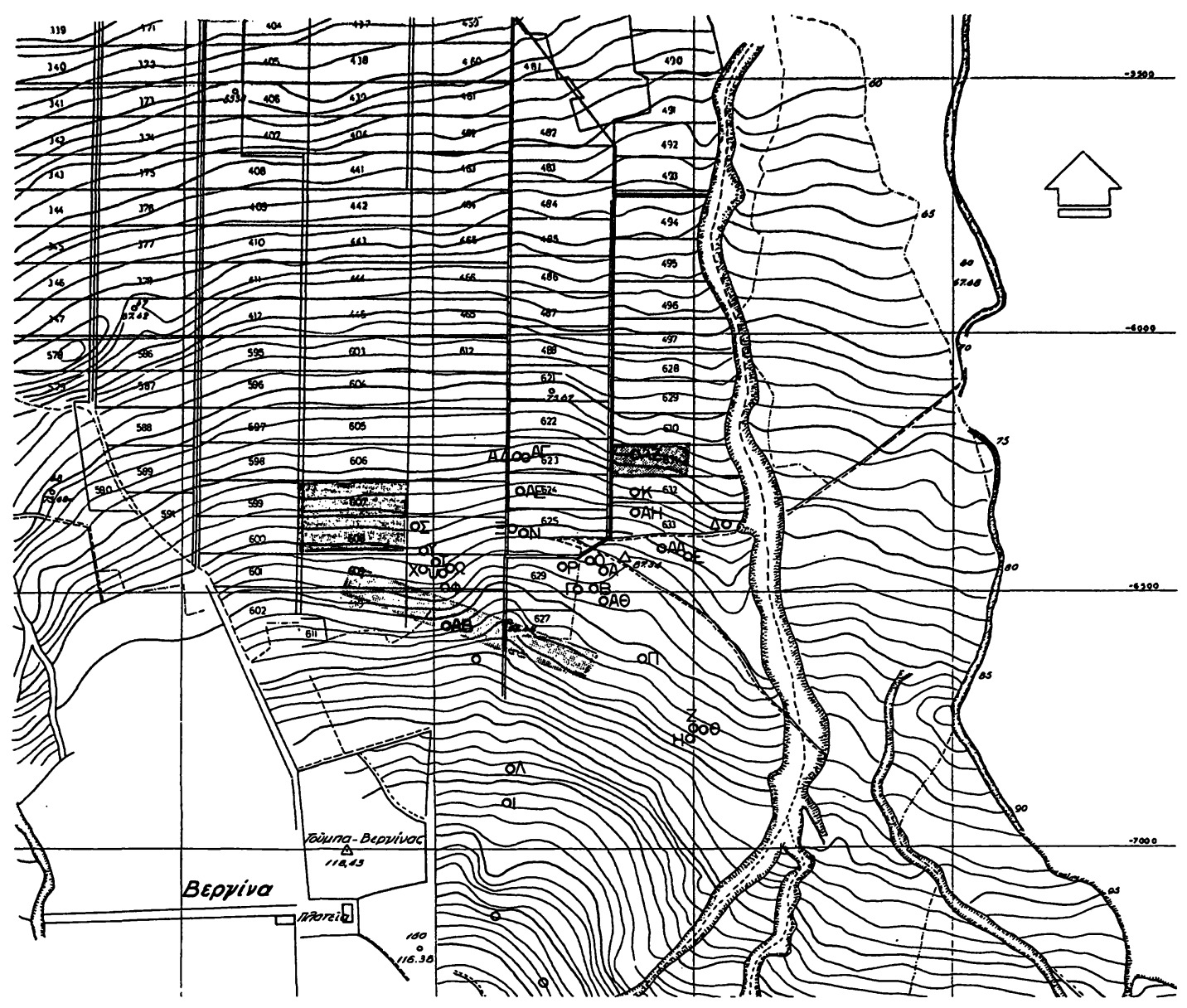

Abb. 1. Vergina. Plan der Nekropole mit den Hügeln der Grabung Andronikos. Helles Raster (im Süden): Grabung Petsas; dunkle Raster (im Westen und Osten): Grabung Rhomiopoulou. Gitterabstände je $500 \mathrm{~m}$

zeichnende Beigabe ist er jedoch nicht, da der gleiche Gefäßtyp im Grab $\Delta V$ mit weiblichem Kopfschmuck kombiniert ist ${ }^{12}$.

Grab II: Rechteckige Grube von ca. $2 \mathrm{~m}$ Länge und ca. 1,10 m Breite; die Grabsohle liegt auf tieferem Niveau als bei den anderen Gräbern dieses Hügels. Über dem Nordostteil der Grube wurde eine unregelmäßige Steinlage angetroffen, die vermutlich im Verlauf eines späteren Eingriffs entstanden ist; dabei dürfte auch dieser Teil des Grabes gestört worden sein. Als einzige Beigabe fand sich nahe der südöstlichen Schmalseite der einhenkelige Becher (a) (Abb. 4,5; 40,d). - Die Form dieses bauchigen, gerieften Bechers ist in Vergina singulär. Der ebenfalls geriefte Becher aus Hügel $A B$ unterscheidet sich

12 Ebd. 17. durch einen deutlich abgesetzten, hohen Zylinderhals ${ }^{13}$. Vergleichbare Formen begegnen allenfalls unter den einhenkeligen Bechern der Gruppe 1 nach Andronikos ${ }^{14}$.

Grab III: Flache Grube von ca. 2,20 m Länge und ca. $1 \mathrm{~m}$ Breite; nahe bei den Schmalseiten je eine Knopfhenkelschale (a-b) (Abb. 4,6.8; 40,c.f). - Die Beigabe von zwei Knopfhenkelschalen und ihre Lage im Grab sind ungewöhnlich. Eine derartige Grabausstattung wwäre wohl eher in protogeometrischer oder noch späterer Zeit zu erwarten ${ }^{15}$

Grab IV: Pithos, zerdrückt, Höhe ca. 1,60 m; auf dem unteren Drittel des Gefäßkörpers plastische Fingertup-

13 Ebd. 188 Abb. 37.

it Ebd. 215 Abb. 55; vgl. auch A. Hochstetter, Kastanas / Die handgemachte Keramik. Prähist. Arch. Südosteuropa 3 (1984) 304.

is Andronikos 1969, 202 f.; Kilian 1975 a, 67. 


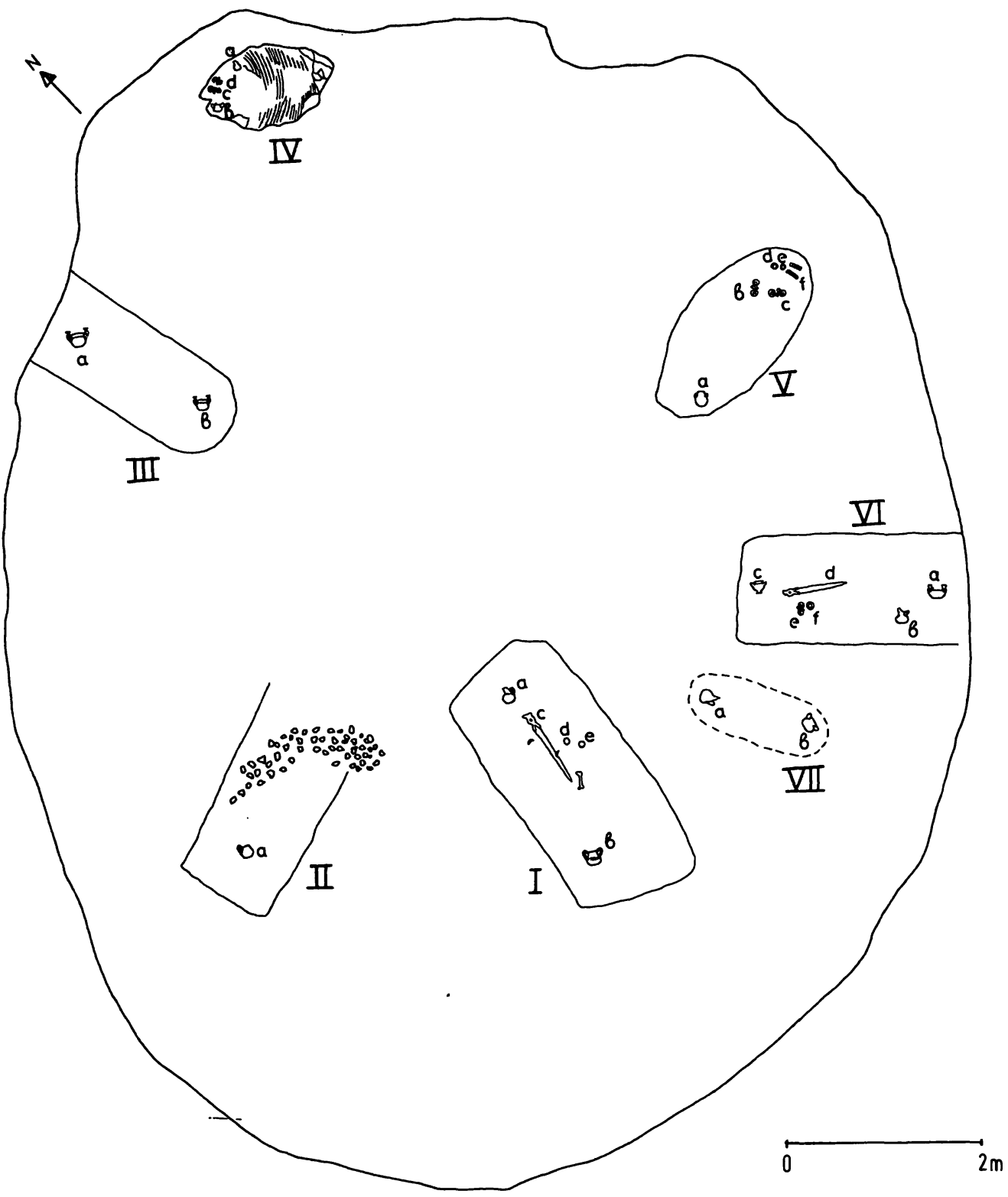

Abb. 2. Vergina. Feld Lazaridis, Grabhügel

fenleiste; Öffnung wahrscheinlich mit einem Stein verschlossen. Nahe beim Boden Amphoriskos (a), Krug (b), Brillenfibel (c) und Fingerring (d) (Abb. 4,4.7; 6,7-8). - Der Pithos lag, wie das in der Nekropole von Vergina mehrfach zu beobachten ist, nahe am Hügelrand ${ }^{16}$. Nach

16 Andronikos 1969, $151 \mathrm{f}$.
Tonbeschaffenheit und Form gehört der Amphoriskos (a) zur Keramik lokaler Produktion".

Grab V: Flache, ovale Grube von ca. $2,20 \mathrm{~m}$ Länge; beim Ostende ein Spiralröllchen ( $f$ ), daneben ein Tutulus (d) mit der Öse nach oben und ein kleiner Bronzering (e),

17 Dazu ausführlicher ebd. $204 \mathrm{ff}$. 


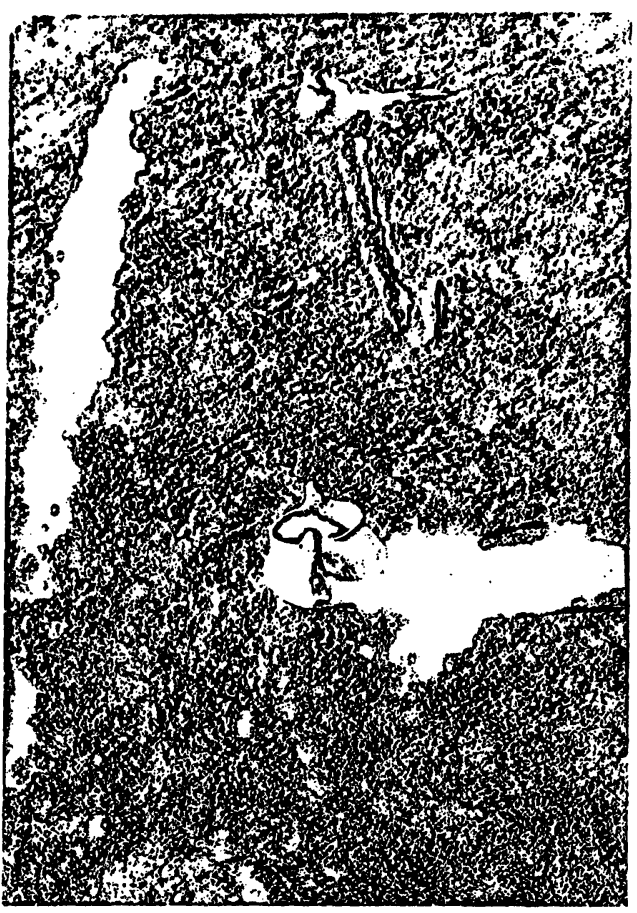

Abb. 3. Vergina. Feld Lazaridis, Grabhügel. Grab I

rechtwinkelig dazu zwei Spiralröllchen ( $f$ ), westlich davon ein Paar Brillenfibeln (b-c); der Amphoriskos (a) am Westende der Grube stand also zu Füßen der Toten. Für zwei zum Inventar dieses Grabes gehörige Eisenmesser $(\mathrm{h}-\mathrm{k})$ und zwei kleine Tutuli $(\mathrm{g})$ konnte die Fundlage in der Grube nicht festgestellt werden (Abb. 5,4; 7,1-14; 41,c-i; 42,d). - Zu dem Amphoriskos (a) aus diesem Grab finden sich in Vergina keine nahen Entsprechungen. $\mathrm{Zu}$ vergleichen wäre allenfalls der Amphoriskos K 23 aus Grab K $I I^{18}$. Durch den Kopfschmuck aus Spiralröllchen wird das Grab Lazaridis $V$ in die Stufe III A-B der Nekropole datiert; gleicher Zeitstellung dürften dann auch die beiden Amphoriskoi sein.

Grab VI $a-b$ : Flache, rechteckige Grube von ca. $1,10 \mathrm{~m}$ Breite; nahe bei der westlichen Schmalseite Skyphos (c); in der Grubenmitte Schwert (d) mit dem Griff zum Skyphos (c), daneben zwei Fingerringe (e-f). 1,50 $\mathrm{m}$ und $2 \mathrm{~m}$ östlich des Skyphos (c) ein Krug (b) und eine Knopfhenkelschale (a). Da diese beiden Gefäße auf tieferem Niveau stehen als die anderen Beigaben, müssen sie zu einem zweiten Grab gehören (VIb), bei dessen Anlage der Ostteil, also das Fußende, der Bestattung mit

18 Ebd. 29 Taf. 41.
Schwert (VIa) gestört wurde (Abb.5,1-3; 6,4-6; 8; 42,a-c; 43,a).

Der Skyphos (c) ist auf der Drehscheibe gefertigt und mit dickem Firnis bemalt. Schlanke Skyphoi auf hohem, konischem Fuß treten in Lefkandi in mittelprotogeometrischer Zeit auf's. In Vergina ist diese Form durch die Skyphoi N 15 und $\Xi 2$ vertreten ${ }^{20}$. Mit der hochsitzenden Schulter und dem abgesetzten Standring zeigt der Skyphos (c) aus dem Grab VIa wohl jüngere Merkmale, so daß er der Stufe III A oder sogar III B der Nekropole zugewiesen werden sollte. - Der Krug (b) aus Grab VIb, der Gruppe 4 nach Andronikos nahestehend ${ }^{21}$, hat einen gedrückt kugeligen Bauch und einen breiten Hals. Vergleichbar ist in Vergina der Krug aus Grab LXVX, der zu einem Inventar der Stufe IIIC gehört ${ }^{22}$. Die Überschneidung der Bestattungen $V I a$ und $V I b$ bedeutet also auch eine klare zeitliche Abfolge.

Grab VII: Sehr flache, unregelmäßige Grube von ca. 1,50 $\dot{\mathrm{m}}$ Länge; die Sohle der Grabgrube lag auf einem etwa $10-15 \mathrm{~cm}$ höheren Niveau als bei den Gräbern $I, V$ und VI. Beim Südende Amphoriskos (a), beim Nordende Krug (b) (Abb. 4,3). - Der Krug (b) mit ausgeschnittenem Nacken ist z. Zt. im Museum nicht nachweisbar. Vom Amphoriskos (a) wurde bei der Grabung nur noch der untere Teil des Gefäßkörpers angetroffen.

Einzelfund: Der große Kantharos (Abb. 5,5; 40,a) lag in der gestörten Oberflächenschicht, so daß er nicht mehr mit einer Bestattung zu verbinden ist. In Form und Ausführung der Riefen gleicht er dem Kantharos (b) aus Grab $I$, von dem er sich lediglich durch Größe und dunklere Färbung unterscheidet.

Wie der Plan Abb. 2 zeigt, sind die Gräber im Hügel radial angelegt; eine Zentralbestattung konnte jedoch nicht festgestellt werden. Das Pithosgrab $I V$ liegt innerhalb des Hügels auf dem gleichen Niveau wie die Grubengräber. Aus dem stratigraphischen Befund ergibt sich also kein Hinweis auf unterschiedliche Zeitstellung der beiden Bestattungsarten ${ }^{23}$. Der Hügel im Feld Lazaridis ist der einzige der Rettungsgrabung von 1970, der geriefte Keramik enthielt. Aus der chronologischen Einordnung der Grabinventare ergibt sich eine Belegungsabfolge, die am Ende der Phase II oder Anfang der Phase III A mit den Gräbern $I$ und $I I$ beginnt, sich mit den Gräbern $V$ und VI a der Phase III A - B fortsetzt, um mit den Gräbern $I I I$ und $V I b$ in der Phase IIIB-C zu enden. Die Pithosbestattung IV läßt sich nur allgemein der Stufe III zuweisen. Die Bestatteten in den Gräbern $I$ und VIa sind schwerttragende Männer, in den Gräbern

\footnotetext{
19 Lefkandi I, 298 Abb. 8,D.

20 Gruppe 4 der Skyphoi nach Andronikos 1969, $170 \mathrm{ff}$. Abb. 24. 21 Ebd. 200 f. (Gruppe 4 der chronologischen Einteilung). .

22 Petsas 1961-62, 277 Taf. 150.

23 Vgl. dagegen Andronikos 1969, $151 \mathrm{f}$, der die Bestattungen in Pithoi generell für später hält als die Bestattungen in Grabgruben.
} 
IV und $V$ weibliche Personen mit einer durchschnittlichen Beigabenausstattung. Da Grab $V$ auch zwei Eisenmesser enthielt, wäre dort eine Doppelbestattung von Mann und Frau zu vermuten. Für die Gräber $I I, I I I$ und VII ist eine archäologische Geschlechtsbestimmung nicht möglich.

\section{Feld Malamas (Parzellen Nr.607 und 608)}

Grabhügel A (Abb.9) (nicht zu verwechseln mit Hügel A der Grabung Andronikos): Wie Petsas bereits früher bei den benachbarten Hügeln $C$ und $C I$ feststellte ${ }^{24}$, war auch der Hügel A weitgehend zerstört. Es besteht also die Möglichkeit, daß er ursprünglich mehr Gräber enthielt, die gegebenenfalls aber durch den Feldbau und andere Eingriffe spurlos beseitigt wurden. Die Gruben der erhaltenen Gräber in diesem Hügel hatten eine Einfassung aus größeren und kleineren Feldsteinen. Von den die Gruben teilweise überdeckenden Steinlagen wurden nur noch Reste in situ angetroffen (Abb. 10).

Grab I: Flache Grube von 2,20 m Länge, Steinfassungen an den Längsseiten teilweise zerstört; die Lage der Gefäße unmittelbar am Rand der Grabgrube ist ebenso wie das Fehlen von Steinen an den Schmalseiten eine Folge tiefreichender Störungen durch den Pflug; bei der Nordecke Schale (a), an der südöstlichen Schmalseite Becher (b) und Krug (c) (Abb. 5,6-8). - Die Kalottenschale (a) gehört zur Gruppe 1 nach Andronikos ${ }^{25}$, die in Vergina meist in Gräbern der Phase III A-B vorkommt ${ }^{26}$. Der Krug (c) kann in die Gruppe 3 nach Andronikos eingeordnet werden ${ }^{27}$. Die Kombination von Krug, Schale und einhenkeligem Becher findet sich nochmals in den Gräbern TI und AГ VIII; im Grab ZIII sind es Krug, Becher und Amphoriskos ${ }^{28}$. Alle drei Gräber enthalten Männerbestattungen mit Waffenbeigabe.

Grab II: Tiefe Grube mit Steinsetzung, im Nordteil Schwert (a) mit Griff nach Norden (Abb. 7,15).

Grab II a: Tiefe Grube, bei deren Anlage der Südteil des Grabes $I I$ gestört wurde; im Nordteil Brillenfibel (a); Fundlage von zwei kleinen Bronzeringen $(b-c)$ und drei Tutuli (d) nicht mehr festzustellen (Abb. 7,16-20).

Wegen der weitgehenden Zerstörung des Hügels A waren bei der Grabung keine stratigraphischen Beobachtungen zu einer zeitlichen Abfolge der Gräber $I$ und II möglich. Auch ihre Beigaben erlauben nur eine generelle Einordnung in die Stufe III. Jünger als die Bestattung mit Schwert (Grab II) ist das Grab II a mit Brillenfibel, Bestattung einer Frau, bei dessen Anlage das Südende von Grab II gestört wurde.

\footnotetext{
24 Petsas 1961-62, $241 \mathrm{ff}$.

25 Andronikos 1969, $207 \mathrm{f}$.

26. Kilian 1975 a, $67 \mathrm{ff}$.

27 Andronikos $1969,194 \mathrm{ff}$.

28 Ebd. 24; 47 f.; 64.
}

Grabhügel B (Abb.11) (nicht zu verwechseln mit Hügel B der Grabung Andronikos): Dieser Hügel, aus reiner roter Erde aufgeschüttet, war bereits stark zerstört; sein Rand zeichnete sich nur noch nach Westen hin streckenweise ab. Die Anordnung und Verteilung der Gräber im Hügel bleibt deshalb unklar. Grab $I$ mit der großen Steinpackung scheint aber nicht das Zentralgrab gewesen zu sein. Streufunde im Bereich des Hügels dürften aus weiteren Gräbern stammen, die durch die Feldbestellung zerstört wurden.

Grab I: Große Steinlage von ca. $3 \mathrm{~m}$ Länge; im Ostteil auf einer kleinen Fläche ohne Steine zwei Brillenfibeln (2.13), zwei goldene Lockenringe (14) und mehrere Spiralröllchen (3.15); beim Westrand auf den Steinen Unterteil eines Kruges (4) (Abb.7,21-24; 12,3). - Nach diesem Befund liegt mit dem Grab I offensichtlich eine Bestattung vor, deren Grabgrube von einer über die Grubenränder hinausreichenden Steinlage bedeckt war. Die geringfügigen Verlagerungen des Kopfschmucks (ein Lockenring, mehrere Spiralröllchen) sind dann auf eine Störung zurückzuführen, die auch Teile der Steinpakkung entfernte. Der Krug (4) auf den Steinen könnte eine nach der Grablege auf das geschlossene Grab abgestellte Beigabe sein. Es ist aber auch möglich, daß er zu einer späteren, über Grab I angelegten Bestattung gehörte, die, auf höherem Niveau befindlich, durch den Pflug völlig zerstört wurde.

Gräber I a und I $b$ : Am Südrand der Steinpackung des Grabes I standen auf gleichem Niveau ein Krug (8), ein einhenkeliger Becher (7), eine Knopfhenkelschale (5) und weiter nach Süden ein zweiter Krug (6). Obwohl keine Grabgruben beobachtet werden konnten, sind aus der Position der Gefäße zwei annähernd parallele, N-S orientierte Gräber zu erschließen, bei deren Anlage die Steinpackung leicht gestört wurde (Abb. 12,1-2.5-6). - Der einhenkelige Becher (7) gleicht den Bechern N 28 und AB 13 der Grabung Andronikos ${ }^{29}$. Becher dieser Form, jedoch mit unterschiedlichem Dekor, begegnen auch in Lefkandi und Marmariani ${ }^{30}$. Die Exemplare von Vergina sind zwar lokal gefertigt, die Gefäßform ist aber aus dem thessalo-euböischen Kreis übernommen, allerdings ohne in Vergina besonders beliebt zu werden. Form und Dekor erlauben eine Datierung in subprotogeometrische Zeit (Phase III B-C der Nekropole).

Grab II: Große, flache Grube von unregelmäßigem Umriß; die Funde 1-11 liegen zwar auf gleichem Niveau, nach ihrer Verteilung können sie aber nicht zu nur einer Bestattung gehören. Der Halsring (7), neben dem sich Perlen (11), Spiralröllchen (10), Tutulus (9) und ein Backenzahn befanden, und die Armspirale (8) sind wohl in situ verbliebene Beigaben einer Kinderbestattung, zu der auch Krug (4) und Skyphos (3) gehörten. -

29 Ebd. 180 f.; Abb. 30; Taf. 44.

30 Lefkandi I, 293 ff. Taf. 174,13.4; Heurtley u. Skeat 1930-31, 26 Taf. 6,b. 


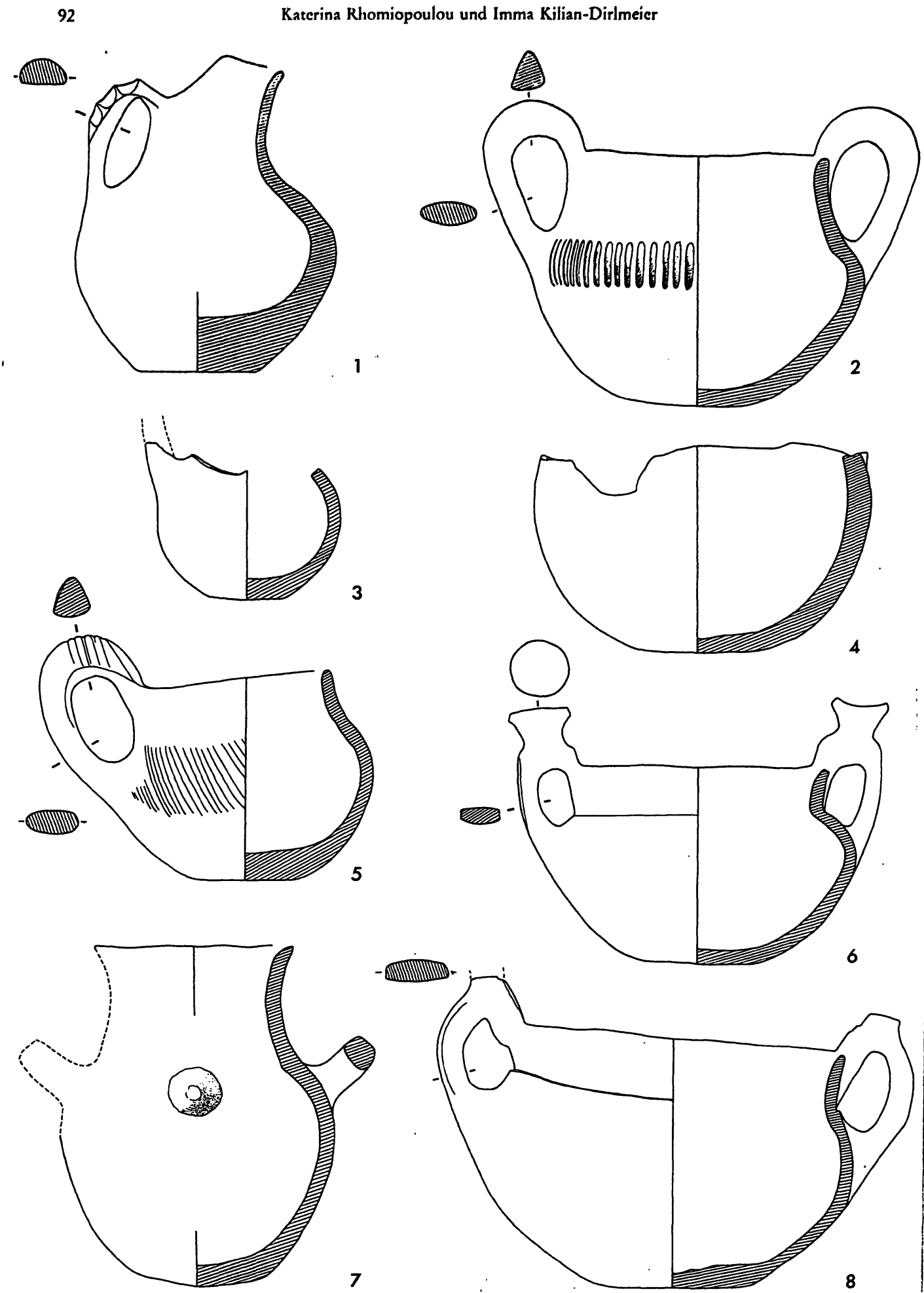

Abb. 4. Vergina. Feld Lazaridis, Grabhügel. 1-2 Grab I; 3 Grab VII; 4.7 Grab IV; 5 Grab II; 6.8 Grab III. - M. $1: 3$

Brought to you by | University of Glasgow Library 


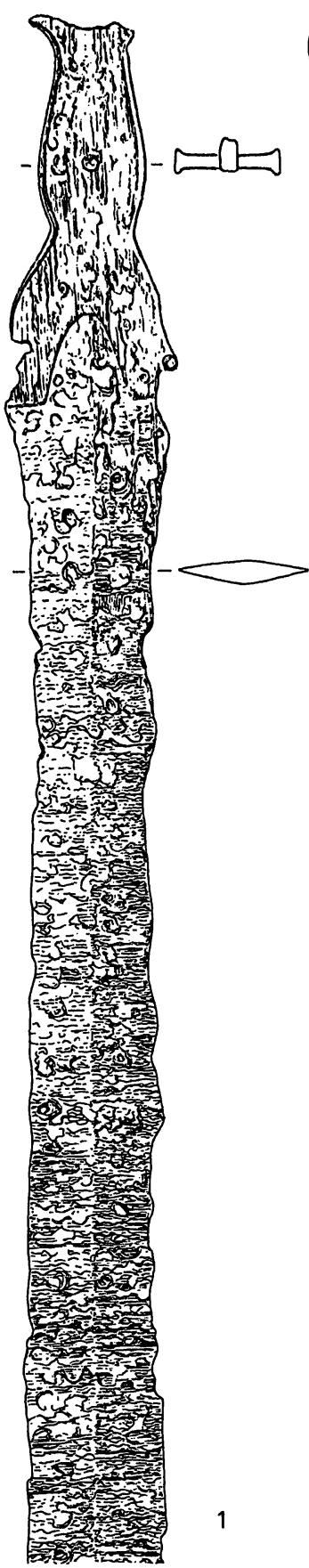

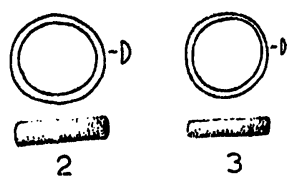
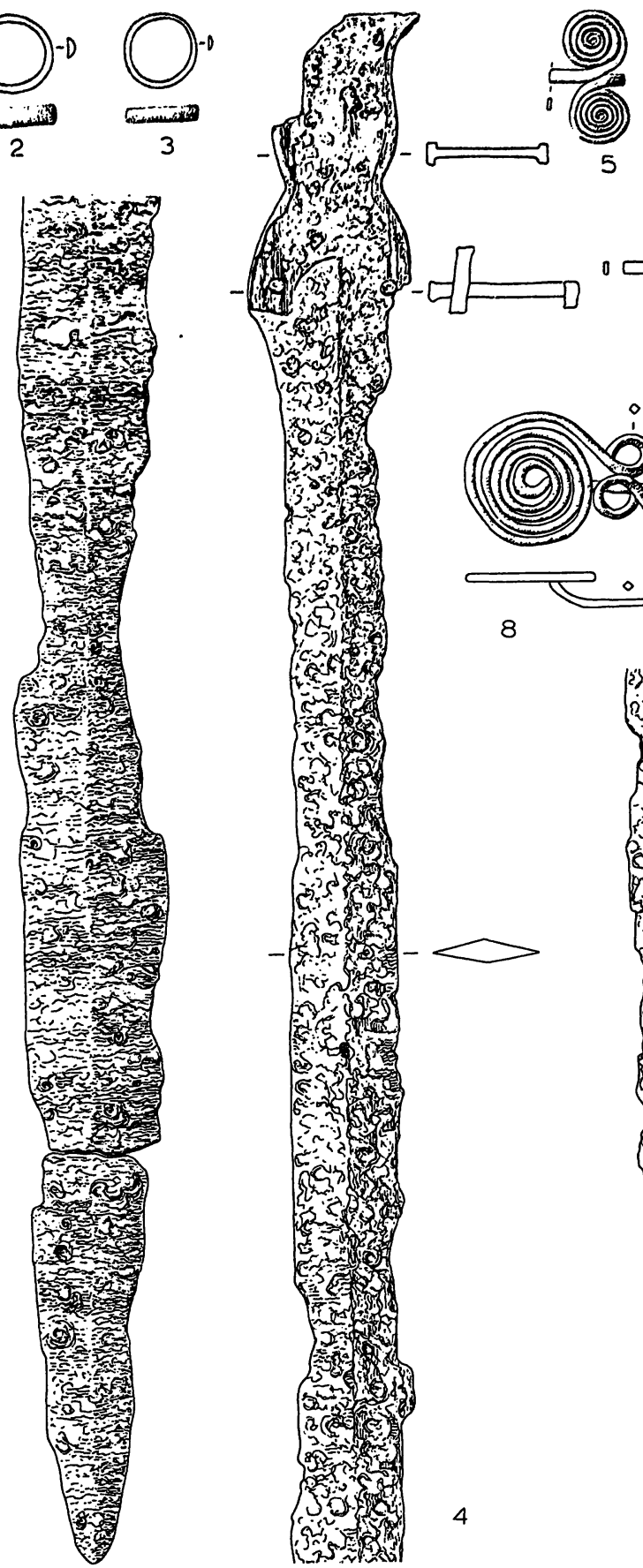
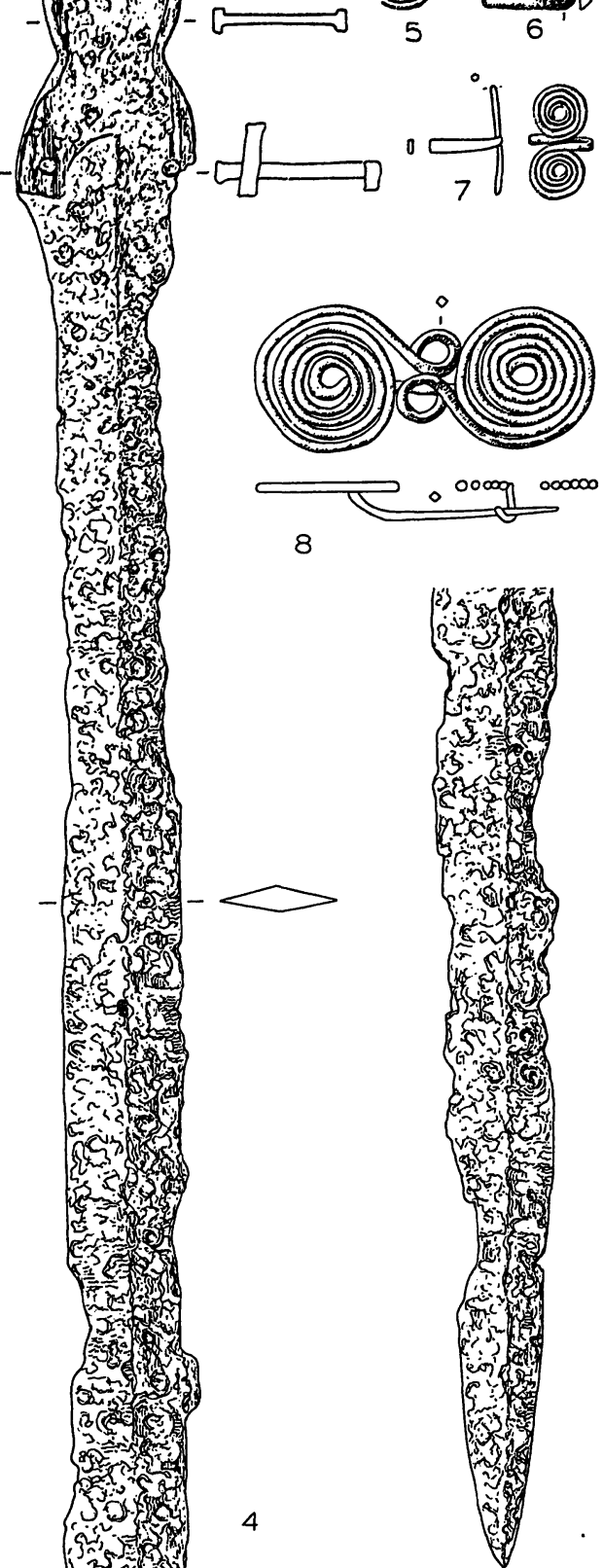

Abb.6. Vergina. Feld Lazaridis, Grabhügel. 1-3 Grab I; 4-6 Grab VIa; 7-8 Pithos IV, - 1.4 Eisen; 2-3.5-8 Bronze. M. $1: 2$ 

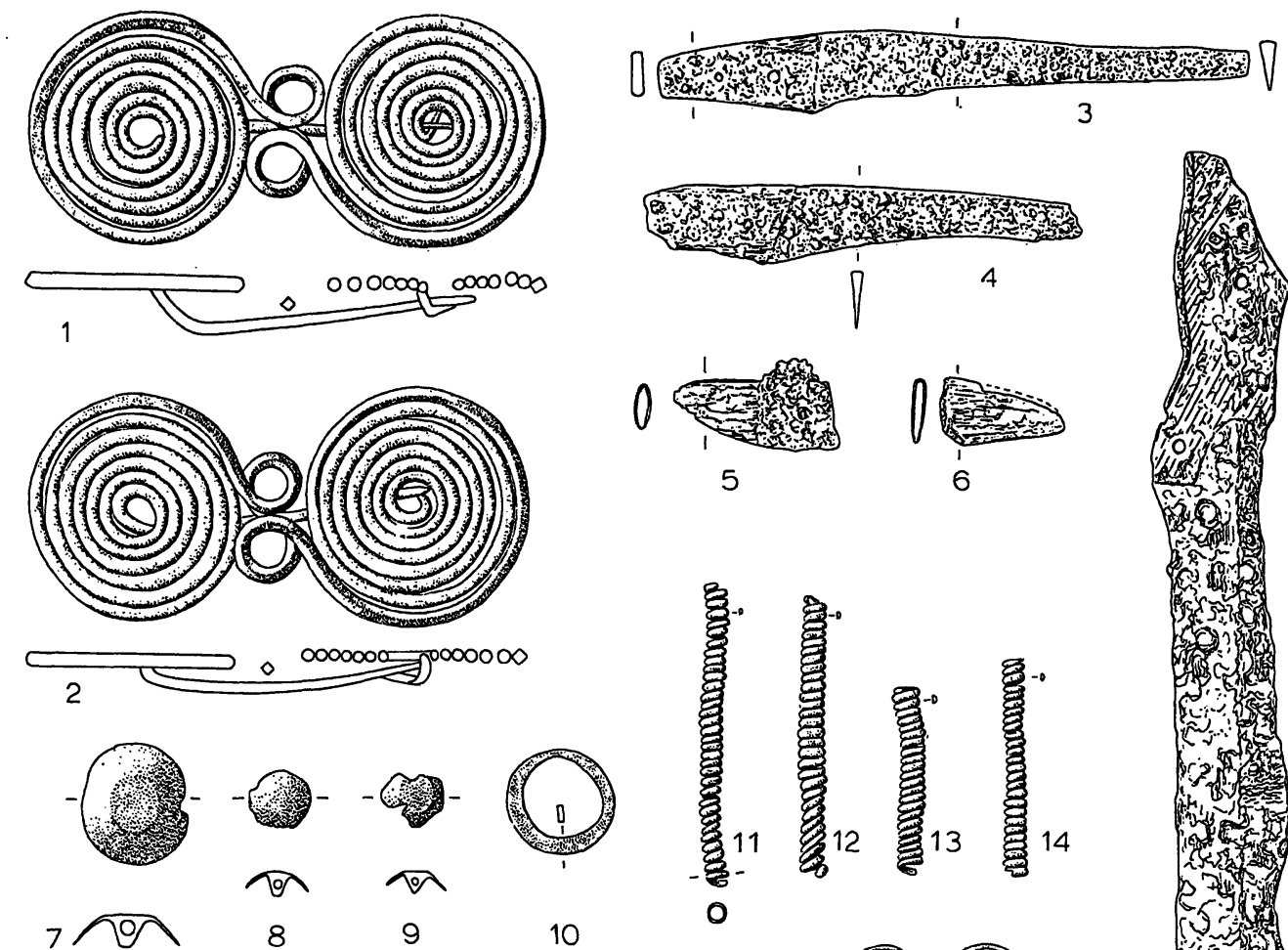

5
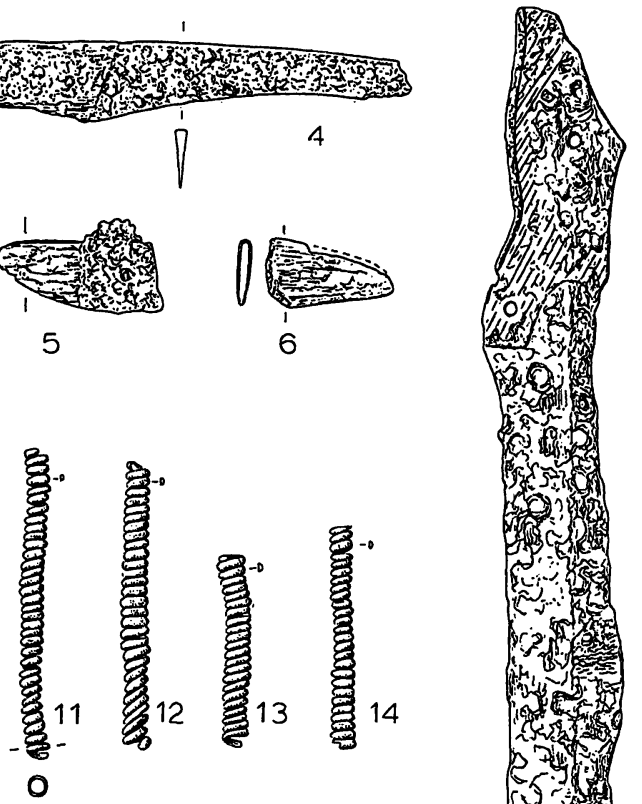

$7 / 8 \quad 8 \quad 9 \quad 10$
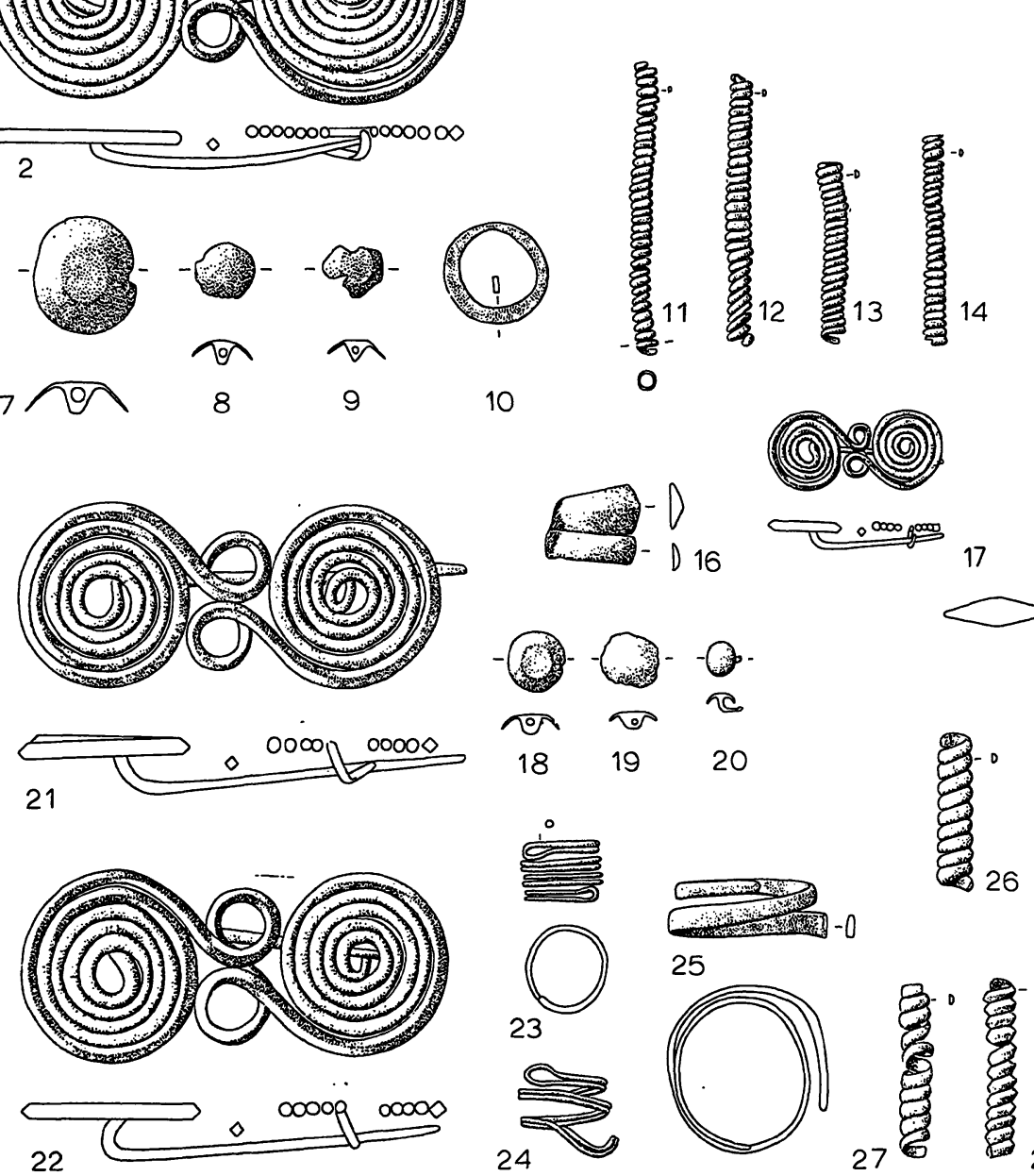

Abb.7. Vergina. Feld Lazaridis, Grabhügel. 1-14 Grab V. - Feld Malamas, Grabhügel A. 15 Grab II; 16-20 Grab IIa. - Feld Malamas, Grabhügel B. 21-24 Grab I; 25-28 Streufunde im Bereich von Grab I. - 1-2.7-14.16-22.25-28 Bronze; 3-6.15 Eisen; 23-24 Gold. - M. 1:2 


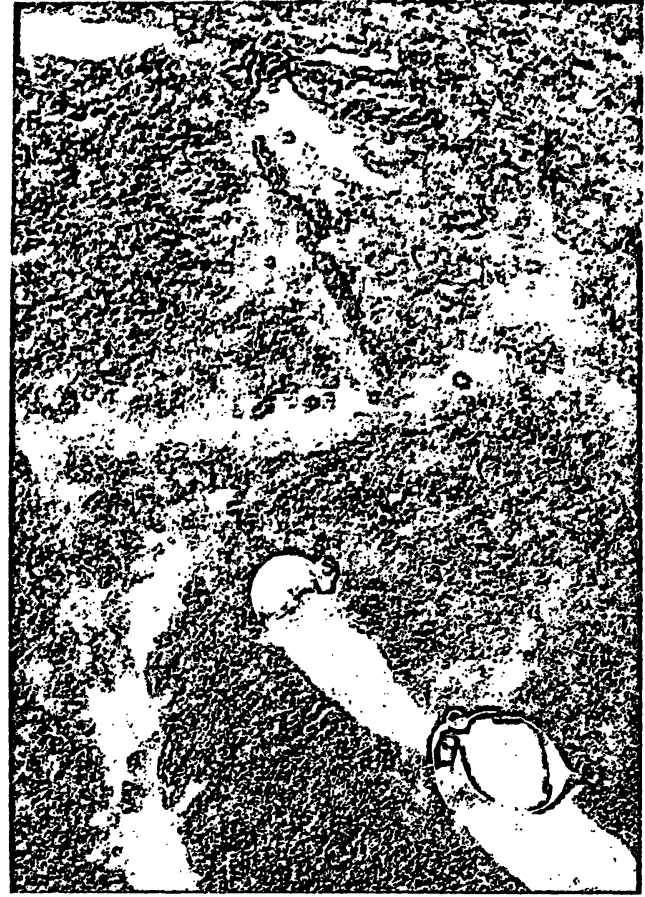

Abb. 8. Vergina. Feld Lazaridis, Grabhügel. Gräber VI $a-b$

Die Knopfhenkelschale (2), etwa 0,60 m vom Krug (4) entfernt, könnte das Fußende dieses Grabes markieren oder zusammen mit dem Krug (1) Beigabe einer zweiten, $\mathrm{O}-\mathrm{W}$ orientierten Bestattung sein. - Krug (5) und Amphoriskos (6) müßten dann von einer dritten Bestattung in diesem Bereich stammen, deren Orientierung nicht zu bestimmen ist (Abb. 12,7-12; 14; 17,1-7; 44).

Die Form des Skyphos (3) ist subprotogeometrisch, sein Dekor (Wellenband) steht jedoch in submykenischer Tradition ${ }^{31}$. Entsprechungen zu diesem Skyphos liegen aus Vergina bisher nicht vor. Nach dem Grabungsbefund kann die Zusammengehörigkeit von Skyphos (3) und Krug (4) nicht zweifelhaft sein. Für den Krug (4), in die Gruppe 3 nach Andronikos einzuordnen ${ }^{32}$, muß damit die gleiche Datierung ins Subprotogeometrische zutreffen. Der Amphoriskos (6) vertritt einen in Vergina noch nicht belegten Typ mit niedrigem, weitem Hals ${ }^{33}$.

Grab III: Flache, unregelmäßige Grube von ca. $1,50 \mathrm{~m}$ Länge. Nach der Zusammensetzung könnten die Funde 1-5 aus dieser Grube als nicht mehr vollständiges Inventar einer Bestattung gewertet werden. Die Fundlage

31 Vgl. Lefkandi I, 297 f. Abb. 8.

32 Andronikos 1969, $196 \mathrm{f}$.

33 Vgl. ebd. $204 \mathrm{ff}$. der Beigaben wärc dann durch eine partielle Störung zu erklärcn, bei der je ein Lockenring (4), Armring (2) und Armspiralc (3) annähernd in der ursprünglichen Position verblieben, während der Kopfschmuck (1) und eine Brillenfibel (5) ans entgegengesetzte Ende der Grabgrube vcrlagert und der zwcite Lockenring, vermutlich auch cinc «wcite Brillenfibcl sowic die Beigefäße entfernt wurden (Abb. 17,8-13).

Grab IV: Sehr flache, ovale Grube von ca. $1,50 \mathrm{~m}$ Länge; am cinen Ende einhenkeliger Becher (1), nahe dabei Messer (2), am anderen Ende Knopfhenkelschale (3) (Abb. 13,1.9; 18,1). - In Form und Oberflächenbehandlung gleicht der Becher (1) dem Becher (b) aus Malamas Hügel A I. Er dürfte in protogeometrische bis subprotogeometrische Zeit zu datieren sein.

Grab V: Großer Pithos (Höhe 1,68 m; Bauchdm. $0,90 \mathrm{~m}$ ) in horizontaler Lage nahe am Rand des Hügels. Die Mündung war mit einer flachen Steinplatte und cinem Feldstein verschlossen, die zusätzlich durch zwei Steine verkeilt waren. Die Wandung des Pithos ist mit zwei breiten, flachen, plastischen Bändern verziert, deren Ränder von Fingertupfenreihen eingefaßt sind. Weder Andronikos noch Petsas bilden einen Pithos dieser Art ab. Im Pithos Krug (1), Knopfhenkelschale (2), Hälfte einer vierhenkeligen Schale (3) und geringe Skelettreste (Abb. 15; 16,5.7-8). - Der Krug (1) gehört zu der späten Gruppe 4 nach Andronikos ${ }^{34}$. Ein später Typ der Phase III C ist auch die Kalottenschale mit vier randständigen Henkeln (3) ${ }^{35}$. Da der Pithos kaum beschädigt (Abb. 15) und sein Inhalt nicht gestört war, muß diese Schale (3) in bereits fragmentiertem Zustand ins Grab gelegt worden sein. Zur Datierung siehe Grab VI $b$.

Gräber VI, VIa und VIb: Sehr flache Grube ohne deutliche Grenzen; die Funde 1-16 müssen zu mehrerẹn Bestattungen gehören. Die Fundlage von Goldringen (11-12) - nahe dabei einige Zähne! - , Brillenfibeln (8-9) und Armringen (6-7) spricht für eine $S-N$ orientierte Bestattung, neben deren Kopf die Knopfhenkelschale (10) stand. Krug (5) und zweihenkelige Schaie (4) im Abstand von ca. $1,60 \mathrm{~m}$ von der Schale (10) könnten dann das Nordende des Grabes VI markieren. - Schale (1) und Skyphos (2) sind zu weit entfernt, um ebenfalls mit diesem $G$ rab verbunden zu werden. Die nahe dabei gefundenen Perlen (3) lassen hier den Kopfbereich einer weiteren Bestattung vermuten (VIb). Bei der Materialaufnahme im Museum lagen in der Schale (1) eine große Scherbe einer weiteren Schale (1 a) und mehrere Scherben eines Kruges ( $1 \mathrm{~b})$. Es konnte nicht mehr geklärt werden, ob diese Scherben bei der Ausgrabung in der Schale (1) gefunden wurden - also Beigaben von Fragmentcharakter waren; vgl. Grab $V$, Schale (3) oder ob sie später bei der Magazinierung hineingelegt wurden. - Eine dritte, gesonderte Fundgruppe (VIa)

34 Ebd. $200 f$.

35 Ebd. 208 f.; Kilian 1975 a, 70. 
bestcht aus Saugkännchen (13), Krug (14), vier kleinen Bronzeringen (15) und einer Perle (16) (Abb. $13,2-8.10-11 ; 17,14-26)$.

Der scheibengedrehte Skyphos (2) wird durch die Form seines Ringfußes in spätprotogeometrische Zeit datiert. Das Winkelband im Schulterfeld entspricht dem Dekor der attischen Vorbilder. Skyphoi mit Winkelband waren bisher aus Vergina nicht bekannt. Gleicher Zeitstellung muß dann auch die unmittelbar neben dem Skyphos (2) gefundene Schale (1) sein. Diese Datierung bestätigt den frühen Ansatz des Schalentyps mit zwei randständigen Henkeln und zwei Grifflappen, den Andronikos vorgeschlagen hat ${ }^{36}$. Saugkännchen gehören zu den selteneren Beigaben in den Gräbern von Vergina ${ }^{37}$; ihre Zeitstellung ist beim Fehlen datierender Beifunde nicht näher zu bestimmen (siehe jedoch unten bei Malamas $\Gamma I V$ ). Bei der Fundgruppe $V I$ a fällt die übereinstimmende Form von Krug (14) und Saugkännchen (13) auf.

Grab VII: Flache, lange, unregelmäßige Grube; die Funde 1-4 können nur als Fundgruppe gewertet werden. Auf etwa gleichem Niveau stehend Scherben einer Knopfhenkelschale (1), daneben Armspirale (1), Schale (2), Krug (3), darin Spirale einer Brillenfibel (3), Schale (4) (Abb. 16,1-4; 18,4-5). - Krug (3) und Schale (4) könnten ihrer Zeitstellung nach zusammengehörige Beigaben eines Grabes sein.

Grab VIII: Teil einer flachen Grabgrube am Nordrand der Steinpackung von Grab I; darin Spiralröllchen (1) und Brillenfibel (3); ein unbeschädigter Krug (1) neben dem Ostrand der Grube ist vielleicht mit dieser gestörten Bestattung zu verbinden (Abb. 12,4).

Unter den Streuscherben aus der Hügelschüttung sind zwei Randfragmente von bemalten Drehscheibengefäßen bemerkenswert: a) Randfragment eines Skyphos mit hängenden Halbkreisen (Abb. 16,9); b) Randfragment eines Skyphos oder Bechers ${ }^{38}$ mit Winkelbanddekor (Abb. 16,6). Ein Vergleich mit den Skyphoi von Lefkandi spricht für eine Datierung nach Subprotogeometrisch I, d. h. in die Phase III B-C der Nekropole, wobei wohl der Skyphos mit Winkelband etwas früher anzusetzen ist, als der mit den Halbkreisen. Im Dekor zeigen beide Skyphoi Nähe zu attischen, nicht zu thessalo-euböischen Vorbildern. Sie dürften beide aus zerstörten Gräbern dieses Hügels stammen.

Die große, die gesamte Grabgrube überdeckende Steinlage über Grab $I$ ist in den Grabhügeln von Vergina einmalig. Bekannt waren bisher einfache Steinfassungen um den Rand der Grube, mit Trockenmauern verkleidete Gruben und Grube mit Steinfassung und Kieselpflaster auf dem Boden. Seiner Lage nach kann das Grab I nicht

36 Andronikos 1969, 209; im Gegensatz zur späten Datierung von Kilian 1975 a, 70.

37 Andronikos 1969, 201.

38 Ebd. $180 f$. als Zentralgrab bezeichnet werden, obwohl es wahrscheinlich die älteste Bestattung dieses Hügels enthält (Phase III A). Die jüngste Bestattung, Grab VI, gehört in die Phase IV A, gleichzeitig oder etwas älter ist Grab III (Phase III C-IV A). Die übrigen Gräber und Fundgruppen gehören der Phase III $A-B$ an.

Grabhügel $\Gamma$ (Abb. 19) (nicht $z u$ verwechseln mit Hügel $\Gamma$ der Grabung Andronikos): Auch dieser Hügel wurde in bereits stark gestörtem Zustand angetroffen. Asche und Brandspuren in den obersten Schichten waren Anzeichen für Bestattungen hellenistischer Zeit. Die Größe und die zahlreichen Streufunde sowohl in der Hügelschüttung als auch auf dem Niveau der Grabgruben lassen annehmen, daß der Hügel ursprünglich noch weitere Gräber enthielt. Zum Teil können die Streufunde aber auch verschleppte Beigaben aus den noch erkenntlichen Gräbern sein. Die Steinsetzungen im Ostteil des Hügels waren Einfassungen von Gräbern. Durch Feldbestellung und Pflügen sind sie stark gestört und wohl auch ihrer Beigaben beraubt. Eine Steinreihe neben dem $P_{i-}$ thos 2 ist vermutlich der Rest eines Steinkreises um den Fuß des Hügels.

Grab I (Abb. 20): Flache, ovale Grube von ca. $1,30 \mathrm{~m}$ Länge; beim Südostende Spiralröllchen (7), zwei Tutuli (8), drei Brillenfibeln (15), kleine Tutuli (17), zwei Lokkenringe (12), Zähne von zwei Individuen, nordwestlich davon Halsring (9), Ringanhänger (10), Drillingsbeil (6), zwei Brillenfibeln (13), Perlen (20), zwei Fingerringe (5), Armspirale (14), Falere (16), kleine Tutuli in Reihen (17), zwei Faleren (16), ca. $20 \mathrm{~cm}$ weiter nordwestlich Radanhänger (18), zwei Faleren (16); neben diesen Trachtbestandteilen Lanzenspitze (2), Schwert (1), Eisenbeschläge (1), sechs Pfeilspitzen (3), Messer (4); im Nordwestteil der Grube zwei Krüge (21.23), Knopfhenkelschale (22) und Kalottenschale (24) (Abb. 21-22; 23,1-4; 25-26; 29,1-27; 45). - Die flachen Drehrillen, Tonfarbe und Oberflächenbehandlung des Kruges (21) finden ihre nächste Entsprechung bei dem Kantharos $\mathrm{Nr} .484$ von Chauchitsa ${ }^{39}$. Es sind Merkmale, die auf eine Datierung in die fortgeschrittene Phase III C der Nekropole verweisen. Die übrigen Gefäße aus dem Grab widersprechen diesem zeitlichen Ansatz nicht.

Grab II: In situ angetroffen wurde nur noch die Schädelpartie einer Bestattung mit Kopfschmuck (2-6), dabei ein nahezu vollständiges Gebiß und ein daneben abgestellter Krug (1) (Abb. 23,5; 27; 30,1-17). - Während der Grabung konnte nicht mehr festgestellt werden, ob das die einzigen Beigaben der Bestattung waren wenig wahrscheinlich - , oder ob die anderen Teile des Grabes zerstört und die weiteren Beigaben verschleppt waren; ursprünglich zugehörig vielleicht die Armspirale XIV (Abb. 18,10) und der goldene Lockenring $a$

39 W. A. Heurtley, Prehistoric Macedonia (1939) 235; Andronikos 1969, 192. 


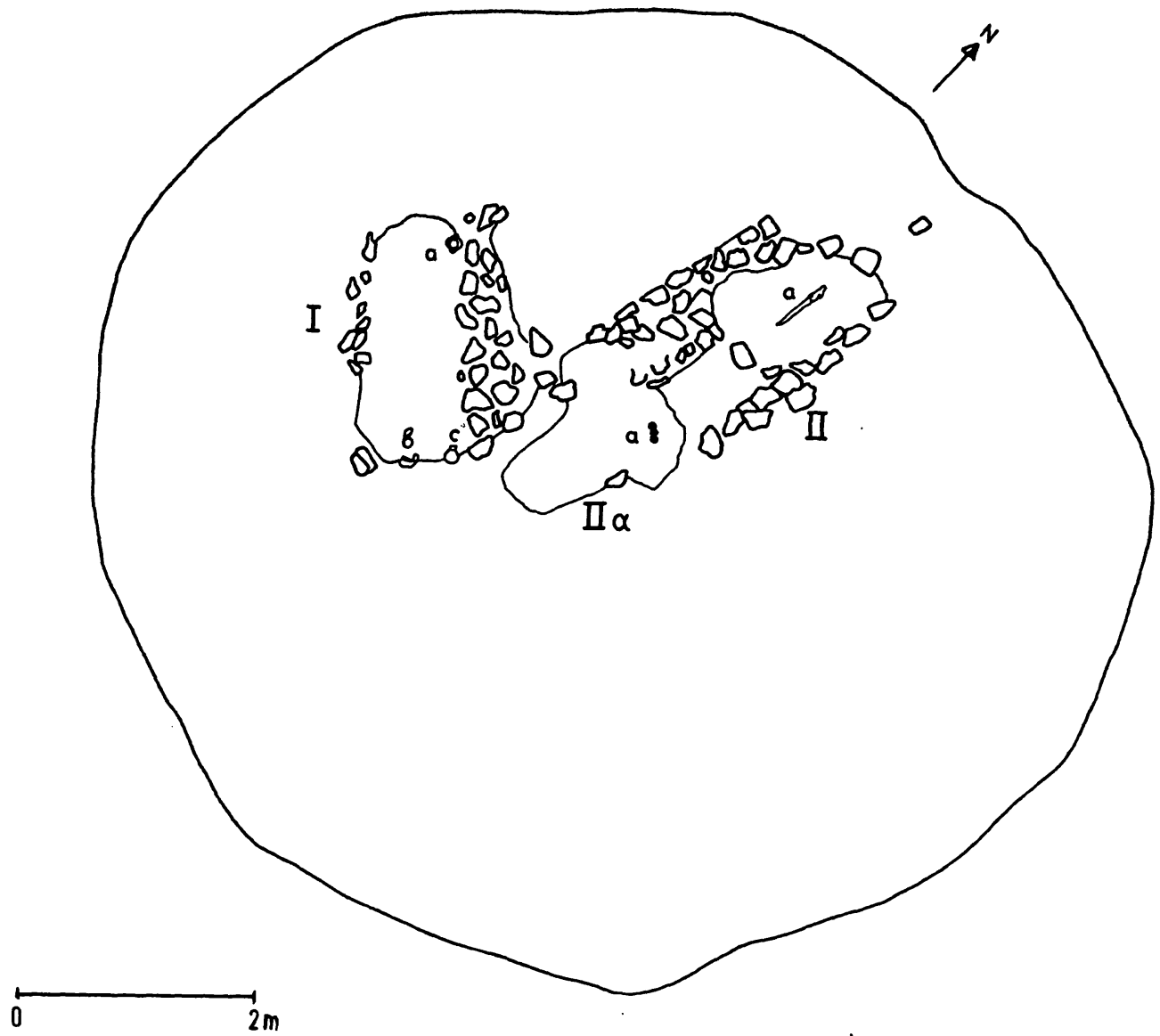

Abb. 9. Vergina. Feld Malamas, Grabhügel A

(Abb. 18,18). Der Krug (1) gehört zur Gruppe 2 nach Andronikos und zwar zu den frühen Exemplaren dieser Form ${ }^{40}$.

Grab III: Flache Grube von ca. 1,20 m Länge; bei der westlichen Schmalseite Krug (1), bei der östlichen Krateriskos (2), dazwischen Schwert (3) mit dem Griff beim Krug (1) hochkant auf der Schneide liegend, daneben zwei Messer (4-5) und Fragmente einer Nadel (6) (Abb. 24,1.3; 28; 30,18-19). - Eine nahe Entsprechung zu dem Krateriskos (2) fand sich in Grab AII von Vergina ${ }^{41}$, in der Siedlung von Kastanas dagegen kommt diese Form nicht vor ${ }^{\wedge 2}$.

to Ebd. 196; vgl. z. B. den Krug A5: ebd. Taf. 30.

41 Ebd. 213 f.; Abb. 54,A22; Taf. 31.

${ }^{42}$ Hochstetter (Anm. 14) $138 \mathrm{ff}$.
Grab IV: Da Saugkännchen (1) und Skyphos (2) nebeneinander stehend gefunden wurden, dürfte es sich um in situ angetroffene Reste eines Grabes handeln und nicht um verschleppte Beigaben aus verschiedenen Bestattungen (Abb. 24,2.4-5; 46,a-b). - Der Skyphos (2) ist ein charakteristisches Beispiel des subprotogeometrischen Stils; in Vergina ist er mit dem Skyphos $\Delta 15$ zu vergleichen ${ }^{43}$. In Thessalien kann allenfalls der Skyphos Nr. 12 von Marmariani als nahestehend genannt werden ${ }^{4+}$. Das Saugkännchen (1) gleicht dem Exemplar aus Malamas B VI a (Nr. 13). Die durch den Skyphos gegebene Datierung in Subprotogeometrisch sollte also auch für jenes Saugkännchen zutreffen.

43 Andronikos 1969, 169 Abb. 23

4 Heurtley u. Skeat 1930-31, 29 Taf. 7,121; Lefkandi i, 301. 


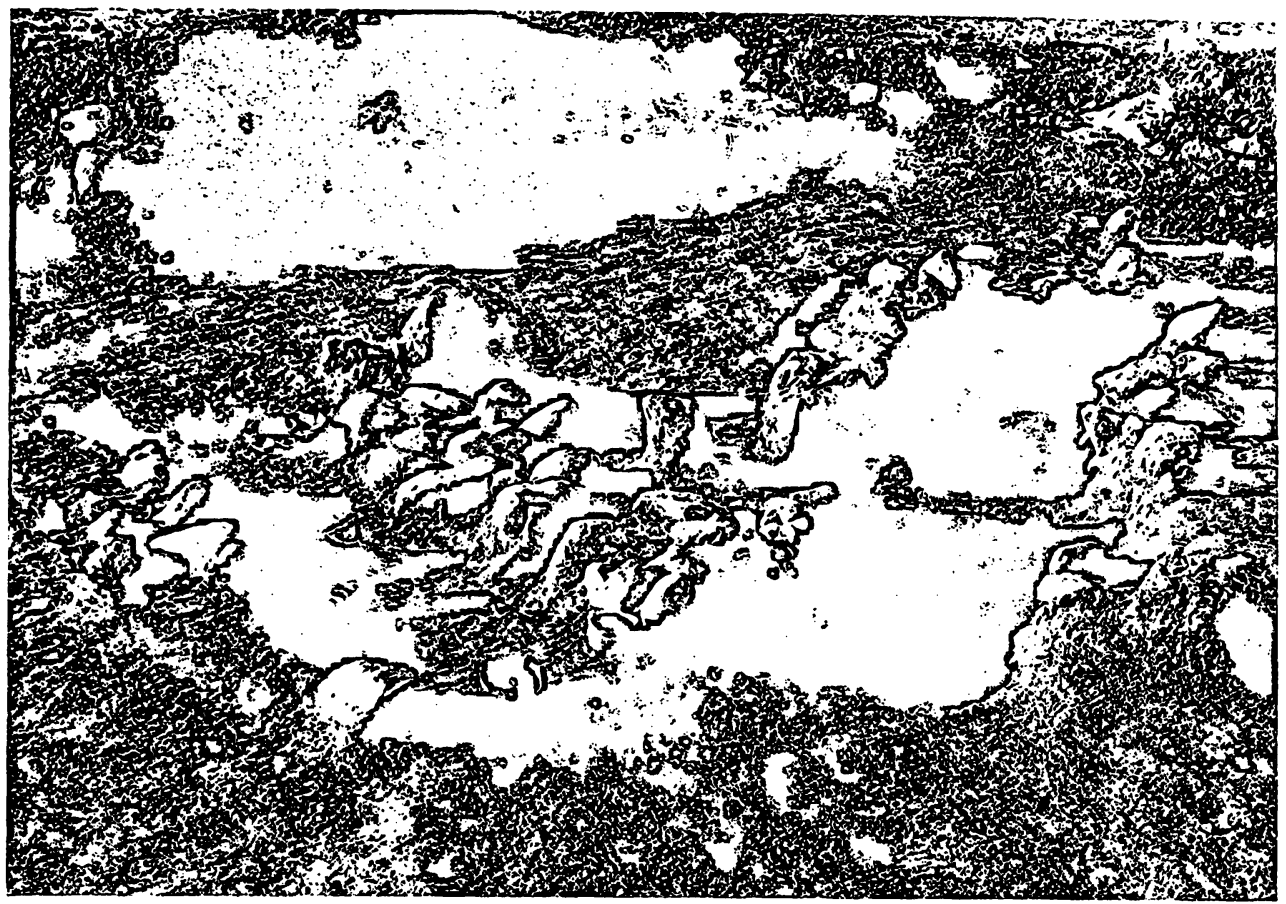

Abb. 10. Vergina. Feld Malamas, Grabhügel A. Gräber $I, I I, I I a$

Grab V: Ovale Grube von ca. 1,20 m Länge; am Südende Knopfhenkelschale (1), am Nordende kleine Brillenfibel (2) (Abb. 18,6; 24,6). - Die Knopfhenkelschale (1) gehört zur jüngeren Form mit kugeligem Gefäßkörpert5; sie fällt durch ihre grobe Machart auf.

Grab VI: Ovale Grube von ca. 1,20m Länge; am Nordende Krug (1), am Südende Skyphos (2); die Ränder beider Gefäße sind durch den Pflug beschädigt (Abb. 31,1.3). - Der Skyphos (2), vom bekannten Typ mit hängenden Halbkreisen, steht dem Skyphos aus Malamas $\Gamma I V(\mathrm{Nr} .2)$ nahe.

Grab VII: Ovale Grube von ca. 1,20m Länge; am Nordende Knopfhenkelschale (1), am Südende nebeneinander Schale (2) und Krug-(3) (Abb. 31,2.4-5). - Ein Geschirrsatz bestehend aus Krug, Knopfhenkelschale und Kalottenschale begegnet nochmals in den Gräbern Malamas B VI (Nr. 4-5.10) und B VII (Nr. 1-3) sowie A $\Gamma X X, \mathrm{LXV} K$ und $\mathrm{LXV} X^{+6}$.

Grab VIII: Ovale Grube von ca. $1,30 \mathrm{~m}$ Länge; am Südende Skyphos (1), am Nordende Topf (2), dazwischen Armring (3) (Abb.29,29; 31,6-7; 47). - Der Skyphos (1) mit hängenden Halbkreisen steht Exempla-

45 Vgl. Andronikos 1969, 202 ff. Abb. 43.

4t Ebd. 66; Petsas 1961-62, 272; 277; Taf. 134, $\gamma ; 137, \alpha$; Kombination von Skyphos, Kalottenschale und Krug im Grab E VIII: Andronikos 1969, 21. ren des thessalo-euböischen Kreises nahe ${ }^{47}$. Durch seine Datierung ins Subprotogeometrische wird auch die Zeitstellung des Topfes bestimmt, der zu einer in Vergina singulären Gattung mit aufgerauhtem Gefäßkörper gehört.

Grab IX: Grube von unregelmäßigem Umriß; am nordwestlichen Ende Saugkännchen (1) und Krug (2), am südöstlichen Ende Knopfhenkelschale (3), einhenkeliger Becher (4) und Skyphos (5), in der Grabmitte Messer (6) und Tutulus (7) (Abb. 18,2-3; 32,1-5; 48). - In Form und Dekor steht der Skyphos (5) thessalischen Exemplaren der protogeometrischen Zeit recht nahe ${ }^{48}$. Ebenso kann das Saugkännchen (1) mit Krugformen aus Marmariani verglichen werden ${ }^{+9}$. Gleicher Zeitstellung muß dann auch der einhenkelige Becher (4) sein, dessen hochgezogener Henkel auf Metallgefäße als Vorbild verweist. Seine gesicherte Datierung ist für die zeitliche Einordnung ähnlicher Becher aus Malamas AI und BIV zu berücksichtigen. Ein Vergleich zwischen den vier Skyphoi aus Hügel $\Gamma$ zeigt zwar ihre generelle Ähnlichkeit, läßt aber auch Unterschiede im Detail erkennen. Anscheinend waren die Töpfer mit Form und Dekor solcher Skyphoi nicht besonders vertraut, so daß die

47 Vgl. Lefkandi 1, $297 \mathrm{ff}$.

48 z. B. Heurtley u. Skeat 1930-31, 29 Taf. 7,120.

4 Ebd. 24 Abb. 9; vgl. auch Lefkandi I, $326 \mathrm{f}$. 

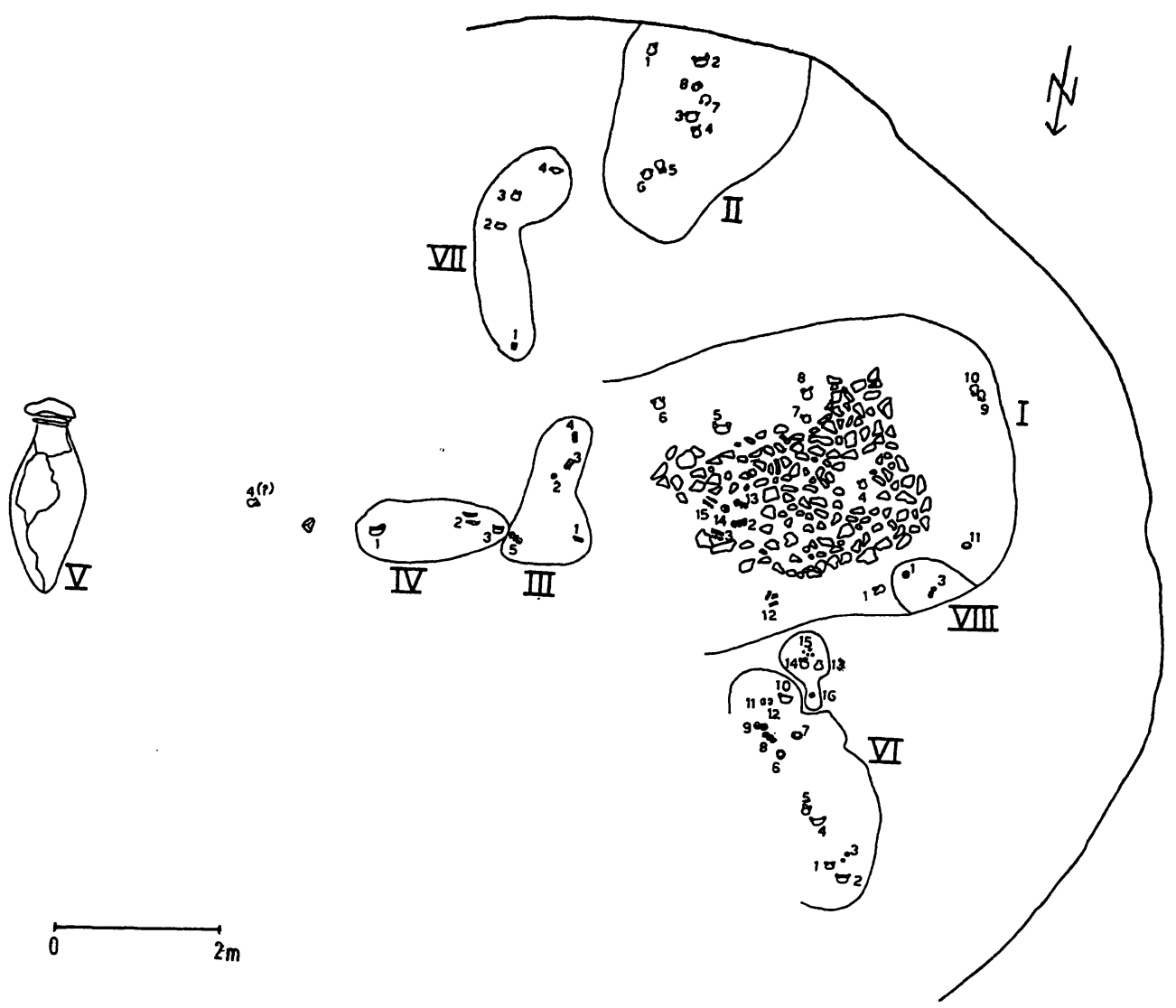

Abb. 11. Vergina. Feld Malamas, Grabhügel B

Ausführung gewisse Unsicherheit verrät. Die Nähe zu den Skyphoi aus Marmariani ist nicht zu übersehen.

Grab X: Ovale Grabgrube von ca. 1,10m Länge; am Westende Krug (1), daneben Perle (4) und nicht mehr identifizierbarer Gegenstand (Spiralröllchen?) (3), am Ostende Knopfhenkelschale (2), Fragment eines Spiralröllchens (3 a) im Krug (1) (Abb. 18,7-8; 32,6-7). Der Krug (1) ist zwar klein, er gehört jedoch nach seiner Form in die Gruppe 4 nach Andronikos. Auffällig ist seine unebene Standfläche.

Grab XI: Viereckige Grube von ca. $2 \mathrm{~m}$ Länge, die Sohle der Grube liegt auf tieferem Niveau als bei den anderen Gräbern; bei der nördlichen Schmalseite Krug (1) und Knopfhenkelschale (2) (Abb. 32,8). - Der Krug (1) zeigt die gleiche Unausgewogenheit von Form und Proportionen, die für die Gruppe 4 nach Andronikos kennzeichnend ist.

Grab XII: Flache Grube von ca. $2 \mathrm{~m}$ Länge; am östlichen Ende Amphoriskos (1), Krug (2) und Knopfhenkel- schale (4), am westlichen Ende Krug (3) und Kalottenschale (5). Der Abstand zwischen den beiden Gefäßgruppen ist so groß, daß an einer Zugehörigkeit zur gleichen Bestattung gezweifelt werden muß (Abb. 33; 34,1-6). Bemerkenswert ist der mit dünnem Firnis aufgetragene Streifendekor des subprotogeometrischen Amphoriskos (1). Möglicherweise handelt es sich um Import, da aus Vergina keine weiteren Beispiele für Amphoriskoi mit Streifendekor bekannt sind ${ }^{50}$.

$X I I I$ und $X I V$ auf dem Plan Abb. 19 sind nur als Einzelfunde zu werten (siehe Katalog).

Grab XV: Der fragmentierte Krug und der Halsring lagen nahe zusammen. Sie gehörten vermutlich beide zu einer zerstörten Bestattung an dieser Stelle (Abb. 29,28; $34,8)$.

so Andronikos 1969, $178 \mathrm{ff}$.; vgl. jedoch den etwas jüngeren Amphoriskos aus Marmariani: Heurtley u. Skeat 1930-31, 26 Taf. 6,82. 
Pithosgrab 1: In dem zerdrückten und nicht mehr vollständigen Pithos lagen ein einhenkeliger Becher (1), Scherben einer tiefen Schale (2) und eine Brillenfibel (3) (Abb. 29,31; 35,1.3). - Der einhenkelige Becher (1) ist das zweite Exemplar dieses Typs aus Vergina ${ }^{51}$. Ähnlich sind ein Becher aus Marmariani und ein Exemplar aus Aiani, das etwas jünger sein dürfte ${ }^{52}$.

Pithosgrab 2: Spitzbodiger Pithos, in Höhe des größten Bauchdurchmessers breites, plastisches Band ${ }^{53}$, Höhe $1,60 \mathrm{~m}$; Bauchdm. $1,20 \mathrm{~m}$; Öffnung mit großer Steinplatte verschlossen; keine Funde.

Die Streu- oder Einzelfunde an kompletten Gefäßen und Scherben (Abb. 35,2.4-13) ordnen sich in den chronologischen Rahmen der Keramikbeigaben aus den Gräbern ein. Der Krug Abb.35,4 und die zwei Schalen Abb. 35,5-6 gehören zu den späten Formen der Nekropole. Unter den Scherben aus der Hügelschüttung sind zwei bemerkenswert: Ein Henkelfragment (von einem Krug?) zeigt Bemalung in brauner, pastoser Farbe (Abb. 35,10) - Mattmalerei? Das Skyphosfragment Abb.35,13 mit schwarz glänzender Firnisbemalung dürfte in Spätprotogeometrisch zu stellen sein.

Der Hügel Malamas $\Gamma$ enthielt im südöstlichen Sektor Gräber mit Steineinfassungen, im Zentrum dagegen Bestattungen in schlichten Gruben. Ein ähnlicher Befund liegt aus Hügel $\Gamma$ der Grabung Andronikos vor; auch dort fand man in den steingefaßten Gräbern keine Beigaben, was Andronikos mit antiker Beraubung erklärtt5. Nach den Keramik- und Metallfunden im Umkreis der Steinsetzungen (Abb. 18,19.25; 35,2.4-9.11-12) sind zumindest im Hügel Malamas $\Gamma$ die Bestattungen in den steingefaßten Gräbern gleichzeitig mit denen in den Grubengräbern. Die Belegungsabfolge in diesem Hügel läßt sich nicht eindeutig bestimmen. Spät, d.h. in die fortgeschrittene Phase III C datiert, ist die Doppelbestattung (Zähne von zwei menschlichen Individuen) des Grabes $I$, während das Schwertgrab III der Phase III B angehören dürfte. Die übrigen Gräber enthalten Keramik, die sich innerhalb der Phasen III A-B nicht genauer einord́nen läßt, so daß es fraglich bleibt, ob die Belegung des Hügels mit dem Schwertgrab III beginnt, oder bereits in der Phase III A mit einem waffenfreien Grab.

\section{Die Kleinfunde, \\ Bemerkungen zu Tragweise und Funktion}

Spiralröllchen

Eine häufige und für die Nekropole von Vergina cha-

51 Andronikos 1969, 219f. Abb.61; vgl. auch den Becher aus Grab III $\Pi$ : Petsas 1961-62, 228 Taf. 103,ع.

52 Heurtley u. Skeat 1930-31, 46 Taf.6,91-92; K. Rhomiopoulou, Annu. Brit. School Athens 66, 1971, 354 ff. Nr. 5; Abb. 2,5; Taf. 58,5.

33 Zur Form vgl. Pithos 3 im Hügel AE: Andronikos 1969, 71 Taf. 26.

st Ebd. 14. rakteristische Beigabe sind die Spiralröllchen. Sie bestehen aus schmalem Bronzeband von dreieckigem bis Dförmigem Querschnitt, das eng gewickelt zu einer festen, kaum elastischen Röhre von etwa $0,8 \mathrm{~cm}$ Durchmesser eingedreht ist; der innere Durchmesser beträgt ca. $0,5 \mathrm{~cm}$, die Längen reichen von $2,7 \mathrm{~cm}$ bis $32 \mathrm{~cm}$. Nach ihrer Fundlage in den Gräbern lassen sie sich eindeutig als Bestandteile eines Kopfschmucks bestimmen, dessen Aussehen und Tragweise allerdings unklar bleiben. $\mathrm{Da}$ die Spiralröllchen für ein Durchziehen einzelner Haarsträhnen zu eng und zu lang sind, hat bereits Andronikos eine unmittelbare Einbeziehung in die Haarfrisur abgelehnt und eine Kopfbedeckung angenommen, an der die Spiralröllchen etwa in Höhe der Schläfen so befestigt waren, daß sie zu beiden Seiten des Gesichts bis auf die Schultern herabhingen ${ }^{55}$. Die Befunde in den Gräbern $I$ und $I I$ vom Feld Malamas, Hügel $\Gamma$, könnten jedoch zusammen mit einigen weiteren der von Andronikos publizierten Bestattungen auf verschiedenartige Tragweisen hindeuten. In regelhafter Wiederholung besteht dieser Kopfschmuck aus zwei Bündeln von Spiralröllchen, zwei Tutuli und einem oder mehreren Ringchen. Da die beiden Tutuli meistens flach und mit der Befestigungsöse nach unten liegend gefunden wurden, ist eine Position seitlich am Schädel, also auf den Schläfen, unwahrscheinlich. Sie müssen in symmetrischer Anordnung zusammen mit den Bronzeringchen die auf der Stirn aufliegende Mittelzier einer Kopfbinde gebildet haben. Die Spiralröllchen hat man sich zweifellos auf Riemen oder Schnüre aufgezogen vorzustellen. Wie ihre in den Gräbern $\mathrm{K} V, \Phi I I I, \mathrm{AB} I, \mathrm{~A} \Gamma V, \mathrm{AE} V, \mathrm{AH} I I, \mathrm{LXV} A P$ und Malamas $\Gamma I, \Gamma I I$ beobachtete Krümmung zeigt, waren sie von den Tutuli ausgehend kranzartig um den Kopf gelegt. Die Aufteilung in zwei Bündel deutet darauf hin, daß diese Kopfbinde im Nacken offen war und mittels der Schnüre gebunden werden konnte ${ }^{56}$. Die Befunde in den Gräbern $\mathrm{N} I X$ und $\mathrm{N} X$ wären dann so zu erklären, daß die Kopfbinde über der Stirn zerriß und die Seitenteile nach außen klappten - daher die Lage der Tutuli mit der Unterseite nach oben ${ }^{57}$. In der Konstruktion gleichbleibend, variieren diese kompositen Kopfbinden

${ }_{55}$ Ebd. $157 \mathrm{f}$; 226.

56 Die ursprüngliche Länge der Spiralröllchen ist in keinem Fall angegeben, nur für Grab AZ VII kann eine Mindestlänge von $17,5 \mathrm{~cm}$ vorausgesetzt werden. Mit einer Länge von ca. $18 \mathrm{~cm}$ könnten die von den Spiralröllchen gebildeten Seitenteile den Kopf nicht ganz umschließen. Komplett erhaltene Stücke $z$. B. aus dem Hügel Lazaridis, Grab $V$ und aus dem Hügel Malamas B, Grab II haben dagegen eine Länge von $7,1 \mathrm{~cm}$ bzw. $2,7 \mathrm{~cm}$. Das kann, muß aber nicht auf unterschiedliche Konstruktion der Kopfbinde hinweisen.

57 Das müßte dann aber auch bedeuten, daß die Toten nicht unmittelbar mit Erde bedeckt waren; vgl. auch Andronikos 1969, 162. 

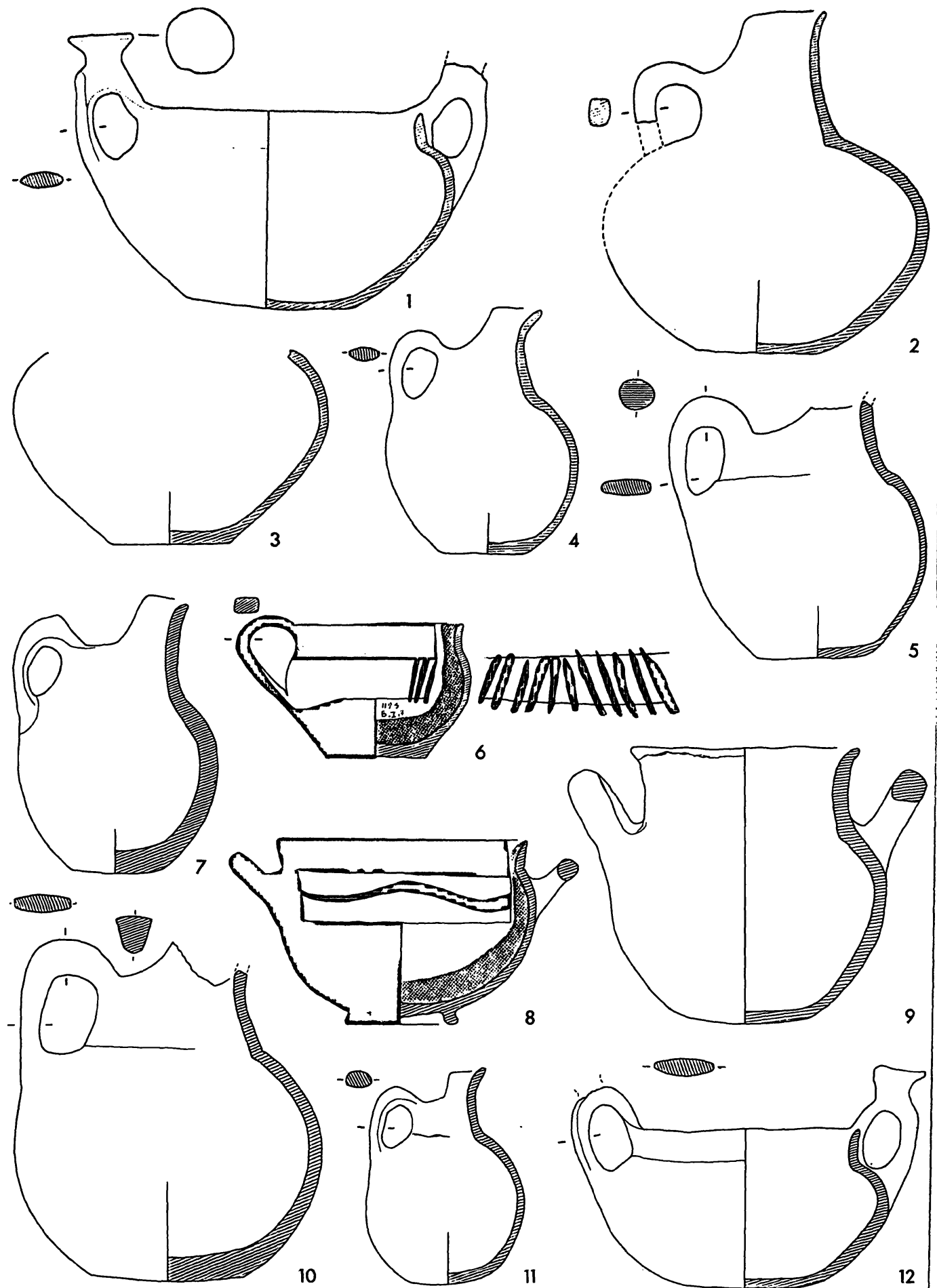

5
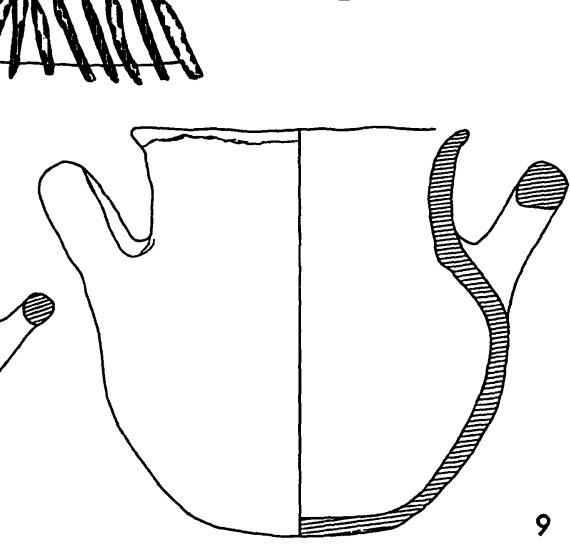

8

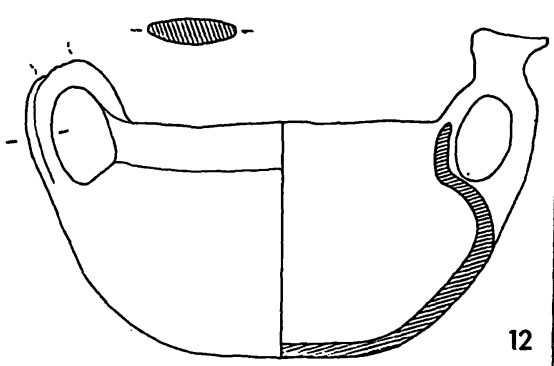

Abb. 12. Vergina. Feld Malamas, Grabhügel B. 1-2 Grab $I b ; 3$ Grab I; 4 Grab VIII (?); 5-6 Grab Ia; 7-12 Grah II. - M. $1: 3$ 


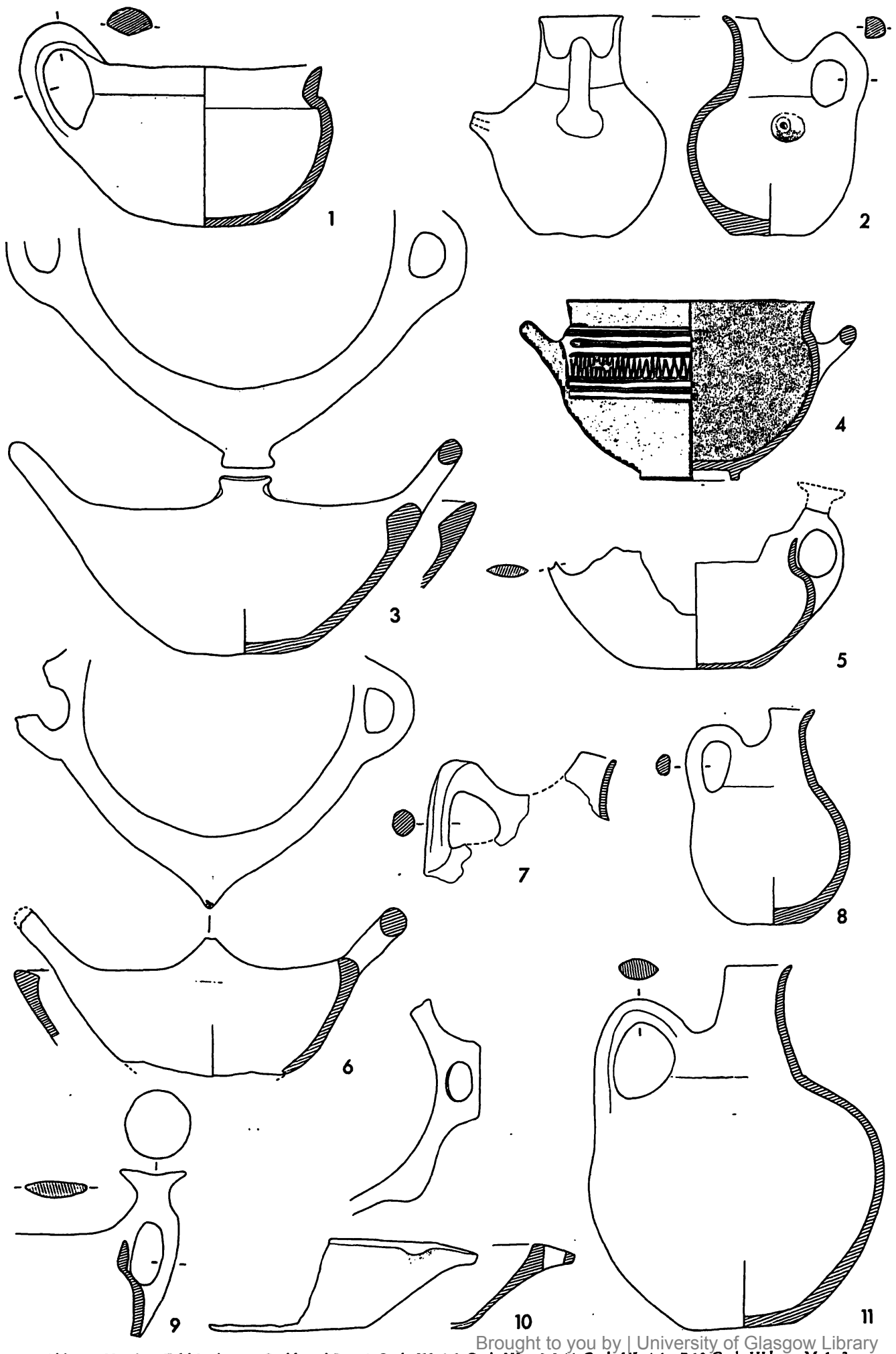

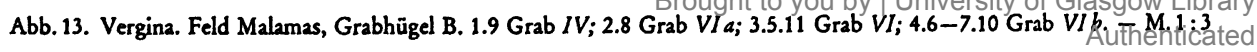




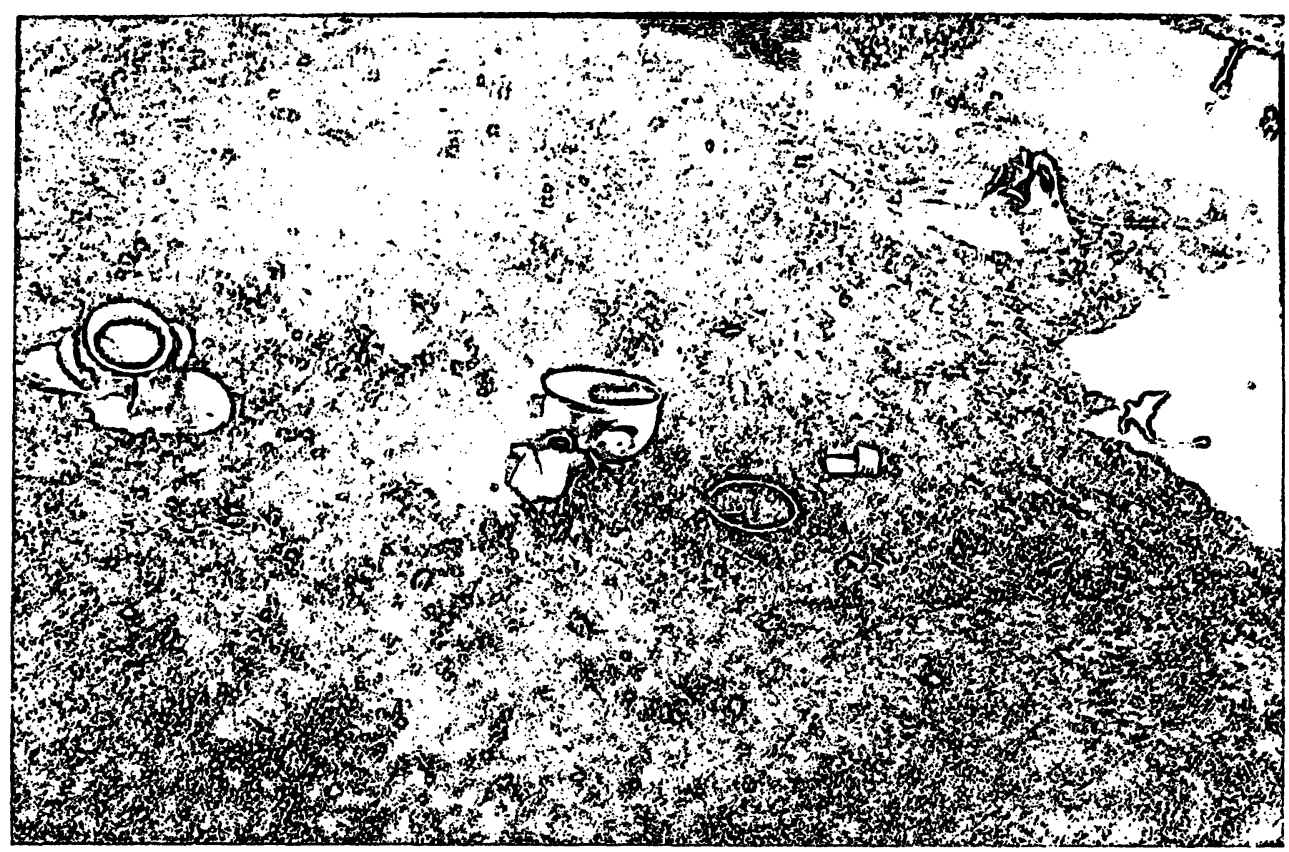

Abb. 14. Vergina. Feld Malamas, Grabhügel B. Grab II

nur in der Zahl der Spiralröllchen ${ }^{58}$. Einen zweiten Typus von Kopfschmuck mit einem zusätzlichen Bündel von Spiralröllchen am Hinterkopf erkannte Andronikos in den Gräbern $A \Gamma V$ und $A Z V I I^{59}$. Die Fundlage im Grab Malamas $\Gamma I I$ (Abb. 27) spricht jedoch eher dafür, eine doppelte Kopfbinde - wohl mit geschlossenem Reif anzunehmen, deren einer Strang die übliche Stirnzier mit Ringchen und Tutuli hat, während beim anderen ein Tutulus im Nacken sitzt. Eine entsprechende Tragweise könnte auch für die Spiralröllchen $\lambda^{\prime \prime}$ mit dem Tutulus $\mu^{\prime \prime}$ des Grabes AZ VII angenommen werden. Außer diesen komplizierten, reich mit Bronzeteilen besetzten Kopfbinden hat es sicher auch einfachere gegeben. Paare kurzer Spiralröllchen (Länge 4-4,5 cm) mit je einem Tutulus an beiden Enden könnten den Mittelbesatz eines gewebten oder geflochtenen Stirnbandes gebildet haben ${ }^{60}$. Eine etwa zur Hälfte mit Spiralröllchen verklei-

s8 Je zwei Spiralröllchen: Gräber E $I, \mathrm{PIV}, \mathrm{T} V, \mathrm{AA} I V, \mathrm{~A} \Gamma V$, LXV AP. - Je drei Spiralröllchen: Gräber $\mathrm{K} V, \mathrm{~N} I V, \mathrm{Y} I$, $\Phi X I V, \mathrm{AB} I, \mathrm{~A} \Delta I, \mathrm{AE} I I I, \mathrm{AE} V, \mathrm{AE} V I I, \mathrm{AZX}, \mathrm{AH} I I$, LXV $\Gamma, \mathrm{LXV} B \Gamma, \mathrm{LXV} B \Theta$. - Je vier Spiralröllchen: Gräber $\mathrm{N} I X, \mathrm{~N} X, \Phi 111$. - Asymmetrische Anordnung mit ein und zwei Spiralröllchen: Gräber $\Delta V I I I, \mathrm{~A} I I I I, \mathrm{AE} I I$. - Asymmetrische Anordnung mit zwei und drei Spiralröllchen: Gräber $\triangle X, O I$.

59 Andronikos 1969, 63; 75; 157.

60 In den Gräbern A IX, P III, AZIV, LXV $\Pi$, Hügel Lazaridis Grab $V$. dete Kopfbinde belegen die Gräber $Z I, \Delta V$ und $\mathrm{A} \Gamma X X$, in denen Tutuli zusammen mit ca. $30 \mathrm{~cm}$ langen Spiralröllchen gefunden wurden. Schließlich kommen in zahlreichen, anscheinend ungestörten Gräbern einzelne, kurze Spiralröllchen vor, deren Anbringung am Kopfschmuck nicht näher zu bestimmen ist ${ }^{61}$.

In der Tragweise vergleichbar, jedoch unterschiedlich konstruiert ist ein diademartiger Kopfschmuck aus Spiralröllchen, der mehrfach in Gräbern des Sarnotales in Unteritalien begegnet ${ }^{62}$. Es sind dort kurze Spiralröllchen von 4-5 cm Länge, die auf Riemen aufgezogen mehrere parallele Stränge bilden; vertikal zwischen die Spiralröllchen eingeschobene, gelochte Bronzestäbchen dienen als Schieber. An den Enden der Stränge konnten zwei Bronzeringchen befestigt sein. Das Diadem wurde im Nacken gebunden und zusätzlich mit Hilfe von kleinen Fibeln an einem Kopftuch befestigt. Eine schlichte Ausführung mit nur zwei Strängen und ohne Schieber fand sich in einem

61 z. B. Gräber N $I, \mathrm{~N} V I, \mathrm{~N} V I I, \mathrm{~N} X I I, \Xi V I, \mathrm{~T} I I, \Phi$ Pithos 2, $\mathrm{AA} I X, \mathrm{~A} \Gamma$ Pithos 3, AE $X, \mathrm{AE}$ Pithos 3, AZI, AZII, AH $V$.

62 S. Marzano sul Sarno, Grab 30: B. D'Agostino, Mélanges d'Archéologie et d'Histoire, Ecole Française de Rome 82, 1970, 575 ff.; Abb. 4-5; 11. - S. Valentino Torio, Gräber 140, 178, 263: P. Gastaldi, Annali Seminario Studio Mondo Classico 1, 1979, 28 Abb. 30,1.3; dies., in: G. Gnoli u. J.-J. Vernant (Hrsg.), La mort, les morts dans les sociétés anciennes (1982) $235 \mathrm{ff}$. Abb. 13. 


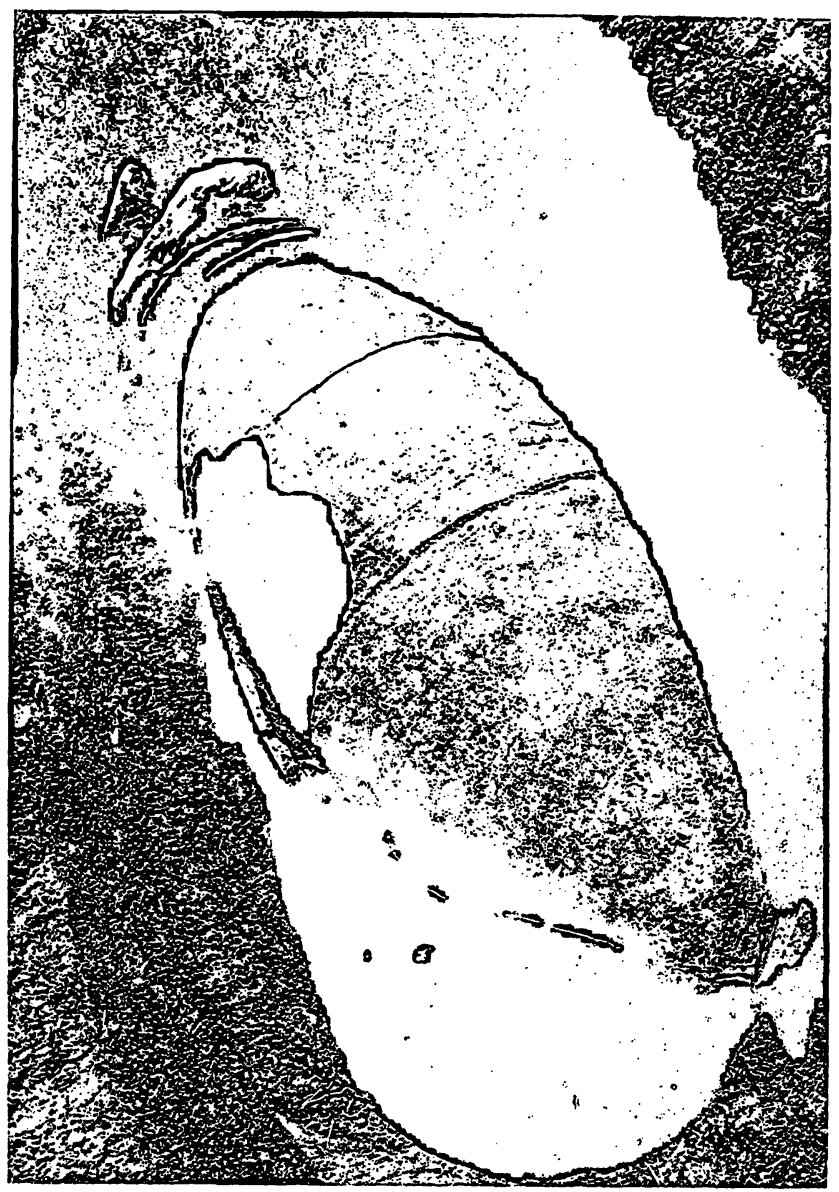

Abb. 15. Vergina. Feld Malamas, Grabhügel B. Pithos V

Kindergrab, während der aufwendige Kopfschmuck zur Frauentracht, zum Teil mit sehr reichem Schmuck gehört. In Vergina wurden die schweren Kopfbinden mit Spiralröllchenbesatz wahrscheinlich, wie schon Andronikos feststellte ${ }^{63}$, über einem-Kopf-Schultertuch aus kräftigem Gewebe getragen. Indizien für ein derartiges über den Kopf gezogenes Tuch sind kleine Besatzbuckel, wie sie z. B. in den Gräbern $\Gamma I$ (Malamas), $\mathrm{A} \Delta I, \mathrm{AE} V$, $A Z V I I$ und bei der Bestattung mit Diadem Y III im Bereich von Kopf und Hals gefunden wurden ${ }^{64}$. Dicht gereiht haben sie wohl eher den Rand des Tuchs gesäumt und nicht ein von Schläfe zu Schläfe unter dem Kinn durchgeführtes Band ${ }^{65}$. Zum Befestigen dieses zusätzlichen Gewandteils sollten dann auch die kleinen Bogen-

63 Andronikos 1969, $257 \mathrm{f}$.

at Ebd. 34 Abb. 6; 52 Abb. 15; 67 Taf. IZ; 69 Taf. IZ; 75 Taf. K. 65 Im Gegensatz zu ebd. $51 ; 75 ; 158$. oder Brillenfibeln gedient haben, die in einigen Gräbern das übliche Paar großer Brillenfibeln begleiten ${ }^{66}$.

Nach den allein zur Verfügung stehenden archäologischen Kriterien (d. h. Länge der Grabgrube, gegenseitiger Abstand der Beigefäße, Armringformat und Fehlen von Waffen) wurde der reiche, komposite Kopfschmuck nur erwachsenen Frauen mit ins Grab gegeben. Einzelne Spiralröllchen dagegen, also schlichte Kopfbinden, finden sich mehrfach in Gräbern, die wahrscheinlich Kinderbestattungen enthielten (z. B. N $I, N V, N V I, N V I I$, $\mathrm{N} X I I, \mathrm{~T} I I, \mathrm{AA} I X, \mathrm{AZ} I, \mathrm{AZ} I I, \mathrm{AH} V)$. Aus Schwertgräbern liegen bisher keine Spiralröllchen vor. Falls sich die Beigaben in den Gräbern III $K$, LXIV $\Gamma$, LXVIII $\Delta$ und LXVIII $E$ nicht auf zwei Tote verteilen - Mann mit Lanze und Frau mit geringem Schmuck -, dann ergibt jedenfalls die Fundlage, daß die einzelnen Spiralröllchen

to So bereits ebd. 158. 


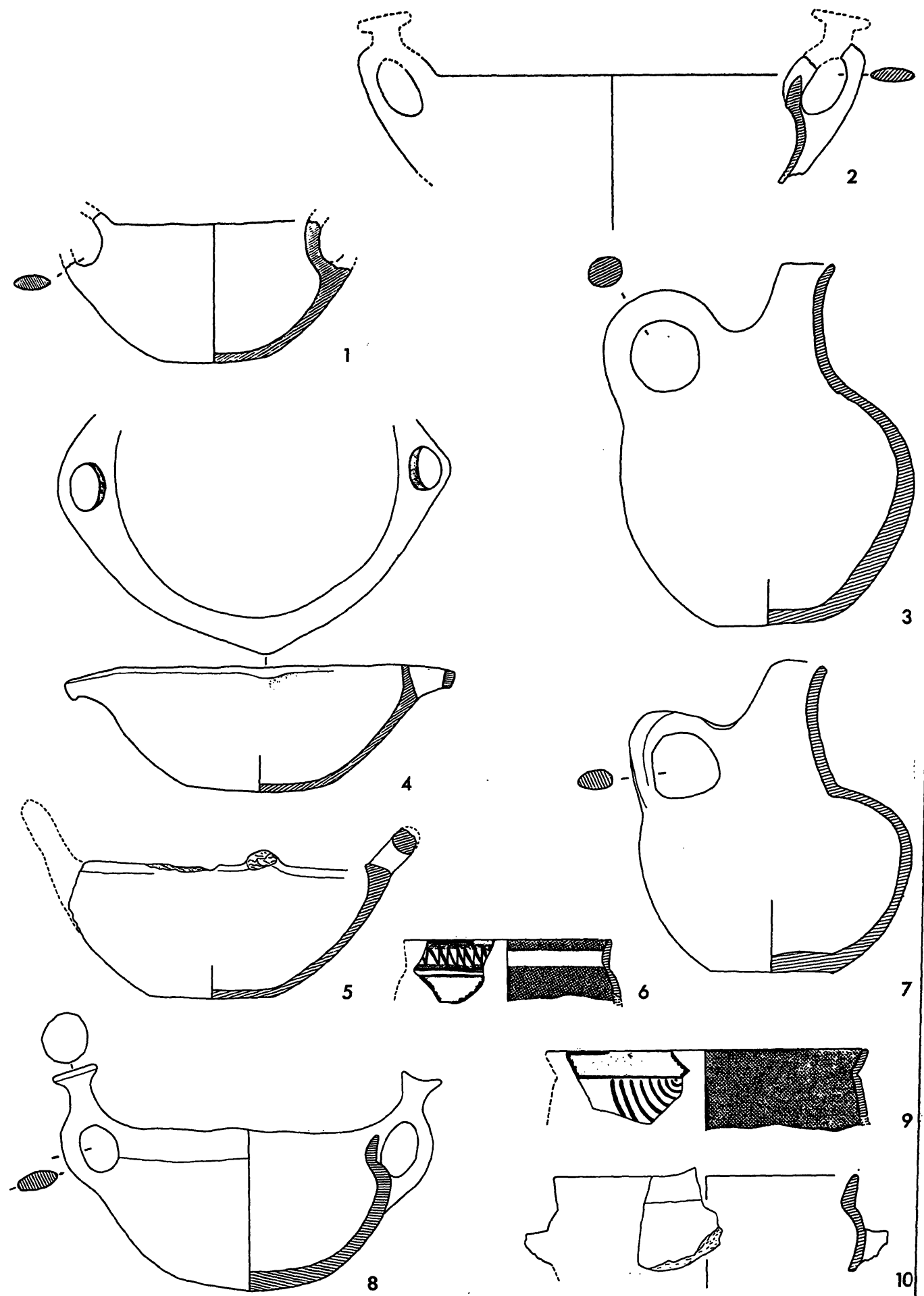

Abb. 16. Vergina. Feld Malamas, Grabhügel B. 1-4 Grab VII; 5.7-8 Pithos V; 6.9-10 Streufunde. - M.1:3 
nicht zum Kopfschmuck der Lanzenträger gehörten. Im unterschiedlichen Aussehen des Kopfschmucks, der in Vergina ein geschlechtsspezifischer Bestandteil der Frauentracht ist, drückt sich wahrscheinlich die Zugehörigkeit zu bestimmten Altersgruppen aus.

Eine komposite Kopfbinde aus Spiralröllchen und Tutuli begegnet in der Nekropole von Vergina erstmals im Grab NX, das durch seine Nadeln in die Stufe II oder spätestens in den Übergang zur Stufe III datiert wird. Alle übrigen Exemplare gehören in die Phasen III A-C. Mit der Stufe IV der Nekropole werden dann die Kopfbinden offensichtlich durch Diademe aus Bronzeblech ersetzt.

$\mathrm{Zu}$ diesem Befund einer klaren zeitlichen Abfolge in Vergina stehen zwei Grabfunde aus Zentral- und Westmakedonien möglicherweise in Widerspruch: Das Grab A von Spilaion im Pindos enthielt ein Diadem aus Bronzeblech mit getriebenem Dekor und zudem Spiralröllchen ${ }^{67}$. Da die Spiralröllchen ebenso wie das Diadem beim Schädel lagen, waren sie vermutlich Bestandteil eines Kopfschmucks. Im Grab 2 von Axioupolis (Bohemitsa) im unteren Axiostal ist die Tragweise der Spiralröllchen nicht mehr zu klären ${ }^{68}$. Sie müssen aber ebenfalls, da sie am Kopfende des Grabes lagen, Teil des Kopfschmucks gewesen sein. Die Glocken- und Kannenverschluß-Anhänger aus diesem Grab sind Typen, die in der makedonischen Eisenzeit II auftreten, also in dem Horizont, dem in Vergina erst die Stufe IV der Nekropole angehört ${ }^{69}$. Der oben zum Vergleich herangezogene Kopfschmuck in Gräbern des Sarnotales gilt als fremder, nicht in der einheimischen Tradition stehender Trachtbestandteil. Bei einer Datierung des Grabes 30 von S. Marzano in die erste Hälfte des 8.Jhs. v. Chr. könnte er Beziehungen zwischen Makedonien und Unteritalien in der Zeit vor der griechischen Westkolonisation anzeigen ${ }^{70}$.

\section{Lockenringe}

Kleine Spiralringe mit einem Durchmesser von $1-2,5 \mathrm{~cm}$ gibt es in Vergina aus Gold- und aus Bronzedraht. Die Goldringe können aus einfachem Draht hergestellt und zu zwei bis fünf Windungen eingedreht sein, oder aus einem doppelten Draht mit schlaufenförmigen, geschlossenen Enden (Abb.7,23-24; 18,13) ${ }^{71}$. Aus Bronze liegen nur einfache Spiralen mit zwei bis vier Windungen vor ${ }^{72}$. Die Zugehörigkeit solcher Spiralringe

\footnotetext{
67 K. Rhomiopoulou, Athens Ann. Arch. 4, 1971, 38 ff. Abb. 1 .

68 L. Rey, Albania 4, 1932, 40 ff.; Abb. 5-6; 8,4.8.

69 Vgl. Kilian 1975 a, 97; Kilian-Dirmeier 1979, 45 ff.

to So K. Kilian, Hamburger Beitr. Arch. 3, 1973, 13.

"Aus einfachem Draht: Grab AIX, NIX, TV, YI, AIX, AE $V, A Z X$, AH $I I$, Malamas $\Gamma$ Einzelfund. - Aus doppeltem Draht: Grab EI, OI, ФIII, A $\triangle I$, AZII, AZVII, Malamas B I, Malamas $\Gamma I$, Malamas $\Gamma$ Einzelfund. - Form unbekannt: Grab LXV $\Gamma, \operatorname{LXV} \Pi, \operatorname{LXV} B \Gamma, \operatorname{LXV} B E, \operatorname{III} \Lambda$.

7 Grab E $I I, \mathrm{Z} I, \mathrm{~K} V, \mathrm{~N} I I I, \mathrm{~N} X, \mathrm{AA} V, \mathrm{AB} I, \mathrm{~A} \Gamma V, \mathrm{AE} I X$, III $A, \operatorname{LXV} A P$.
}

zum Kopfschmuck ist durch die Befunde in den Gräbern gesichert: Es sind meist zwei, die etwas oberhalb der Schultern rechts und links neben dem $\mathrm{Hals}$ liegen; Inventare mit einem oder mit drei Spiralringen begegnen selten ${ }^{73}$. Da sie ihrer Form nach als Ohrschmuck wenig geeignet erscheinen, kommt bei dieser Position eigentlich nur noch eine Verwendung als Lockenring in Frage. Mit den verhältnismäßig geringen Durchmessern waren die Spiralen wohl zu klein, um das gesamte Haar einer Kopfseite durchzuziehen; sie könnten also auf beiden Seiten des Kopfes je eine Haarsträhne zusammengehalten haben, die dann offen oder geflochten über die Schulter herabhing ${ }^{74}$.

Spiralen aus Golddraht mit der Funktion von Lockenringen sind auch in Mittel- und Südgriechenland gebräuchlich. Sie begegnen dort in den beiden Ausführungen in einfachem bzw. doppelt geführtem Draht in Gräbern der submykenischen bis geometrischen $\mathrm{Zeit}^{75}$. Für den goldenen Spiralring aus dem Kammergrab 61 von Mykenai und die drei Paare goldener Drahtspiralen aus dem Schatzfund von Tiryns ergeben zwar die Fundumstände keinen Hinweis auf die Verwendung ${ }^{76}$, mit ihnen sind aber die zwei Typen von Spiralringen bereits in mykenischer Zeit nachgewiesen" ${ }^{7}$. In Vergina gehören die goldenen und bronzenen Lockenringe vorwiegend $\mathrm{zu}$ Grabfunden der Phasen III A-C; ein einfacher Spiral-

73 Zwei Exemplare: Grab AIX, E I, ZI, N III, NIX, NX, OI, $\mathrm{Y} I, \mathrm{~A} \Gamma V, \mathrm{AE} V, \mathrm{AE} I X, \mathrm{LXV} \Gamma$, Malamas B $I$, Malamas ГI. - Ein Exemplar: $\mathrm{E} I I, \mathrm{~K} V, \mathrm{~T} V, \mathrm{AB} I, \mathrm{~A} \Gamma X$. - Drei Exemplare: Grab $\Phi I I I, \mathrm{AA} V, \mathrm{~A} \Delta I$.

${ }^{74}$ Beim Fehlen bildlicher Darstellungen sind Aussagen über Haarlänge und Frisur der eisenzeitlichen Bevölkerung von Vergina kaum möglich. Wenn allerdings Andronikos 1969, 226; 259 annimmt, daß die Lockenringe dazu dienten, das Ende geflochtener Zöpfe zusammenzuhalten, dann ist dies bei ihrer Fundlage in Höhe des Halses eher unwahrscheinlich.

75 z. B. F. Noack, Athen. Mitt. 32, 1907, 557 Abb. 35; A. Bruckner, Athen. Mitt. 51, 1926, 136 f. Beil. 6,1; Kraiker u. Kübler 1939, 39; 87; 97 f.; 183 Taf.76; Kübler 1943, 25; 33 Taf. 39; S. Wide, Athen. Mitt. 35, 1910, 29ff.; Abb. 25; 26; 28; R.S. Young, Hesperia 18, 1949, 275 ff. Nr. 25; Taf.72; N. Verdelis, Athen. Mitt. 78, 1963, 28 ff.; 31 f.; Beil. 11,3.5; R. A. Higgins, Brit. Mus. Quarterly 23, 1960-61, 101 ff. Nr.4-17; Taf. $44 \mathrm{a}, 1-3.5-7$; L. Marangou, Bull. Corr. Hellénique 99, 1975, 367 Abb. 7; 373 Abb. 15; G. Daux, Bull. Corr. Hellénique 91, 1967, $846 \mathrm{ff}$. Abb. 33; P. Courbin, Tombes géométriques d'Argos (1974) 52 ff.; 133 Taf. 36.

${ }^{76}$ Mykenai, Kammergrab 61, Spirale aus dickem Golddraht mit fünf Windungen, Dm. $3 \mathrm{~cm}$ : A. Xenaki-Sakellariou, Oi thalamotoi taphoi ton Mykenon (1985) $186 \mathrm{f}$. Taf. 80. - Tiryns, Schatzfund, sechs Spiralen aus doppeltem Draht, davon zwei mit vier Windungen, Dm. $6,3 \mathrm{~cm}$ und $6,5 \mathrm{~cm}$; zwei Exemplare mit drei Windungen, Dm. $4,2 \mathrm{~cm}$; zwei Exemplare mit 11/2 Windungen, Dm. $2,7 \mathrm{~cm}$ und $2,8 \mathrm{~cm}$ : G. Karo, Athen. Mitt. 55, 1930, 128 Beil. 32; A. Hartmann, Prähistorische Goldfunde aus Europa II (1982) 32 f. Taf. 105, Au 4392 (Materialgruppe P/PC).

7 Zur Datierung siehe K. Kilian, Prähist. Zeitschr. 60, 1985, 159; H. Matthäus, Die Bronzegefäße der kretisch-mykenischen Kultur. PBF II 1 (1980) $56 \mathrm{ff}$. 

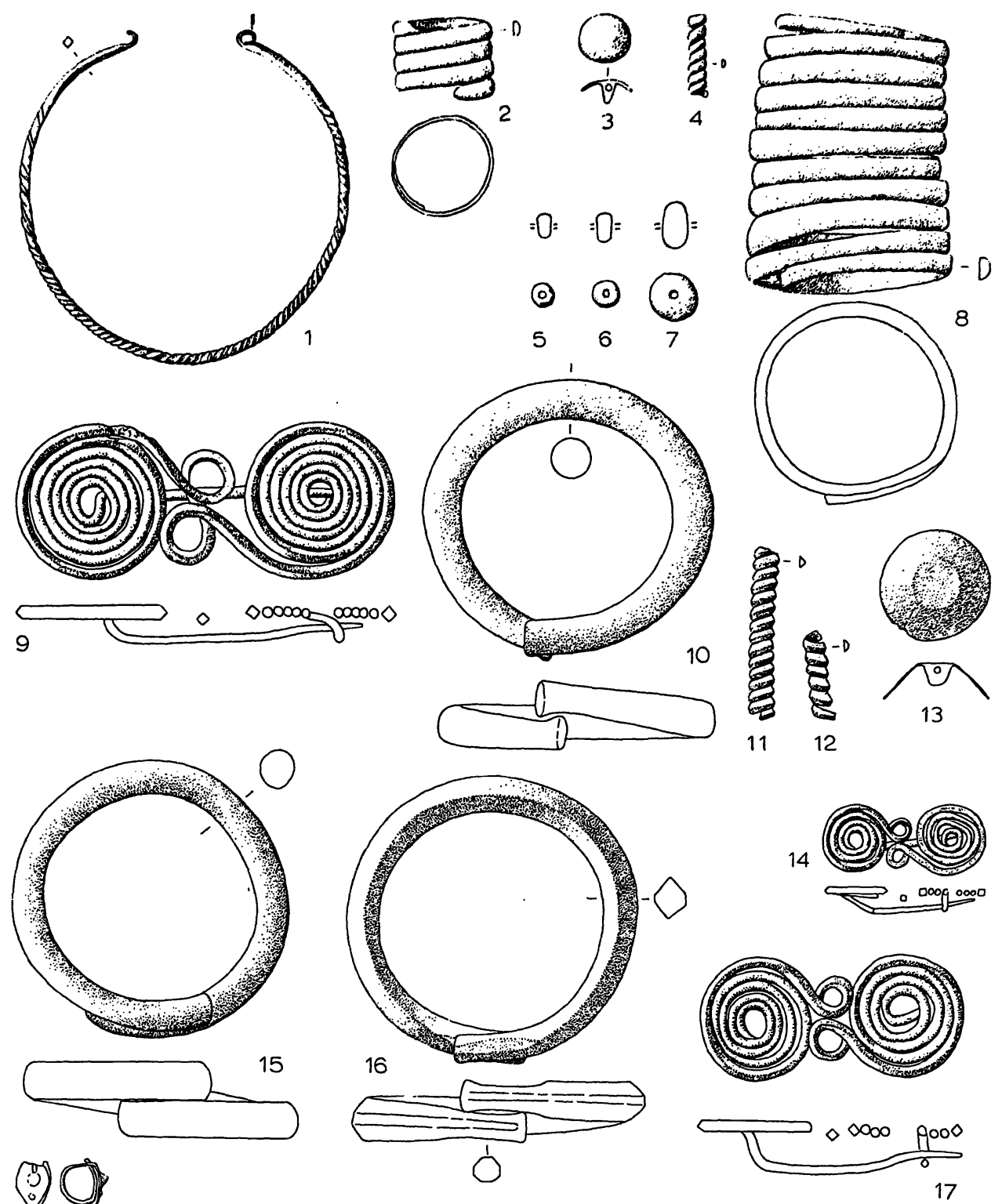

(j)

18

19
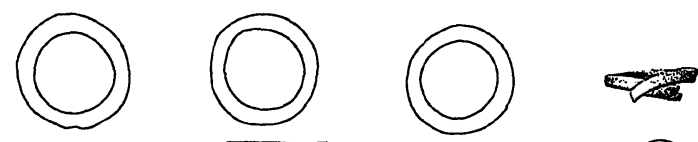

(3)
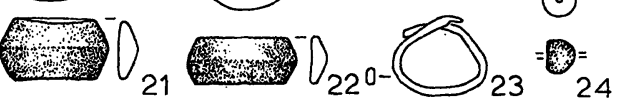

(0)

()

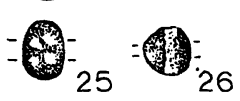

Abb. 17. Vergina. Feld Malamas, Grabhügel B. 1-7 Grab II; 8-13 Grab III; 14-19 Grab VI; 20-24 Fundgruppe VI a; 25-26 Fundgruppe VI b. - 1-4.8-17.20-23 Bronze; 5-7.24 Sard; 18-19 Gold; 25-26 Glaspaste. - M. $1: 2$ 

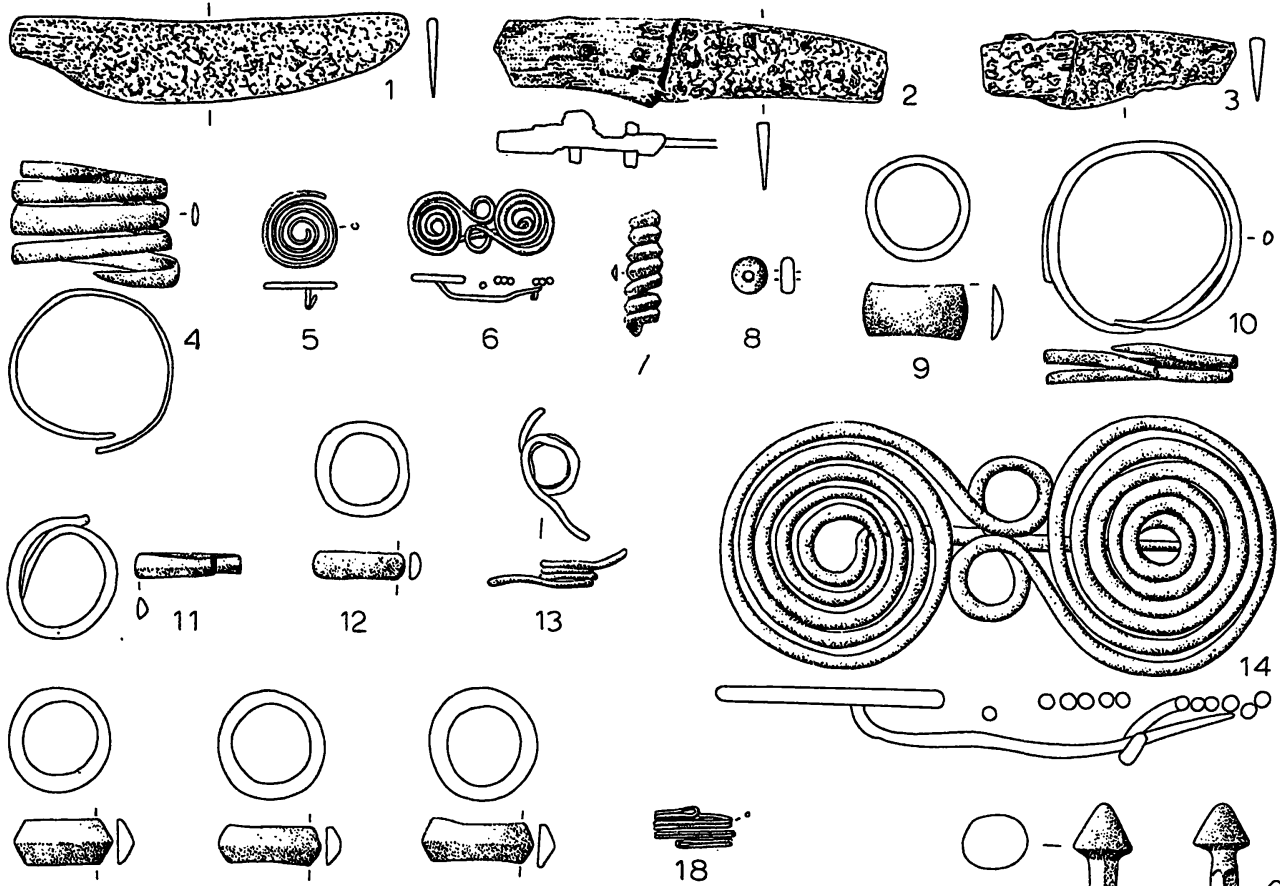

15

16

17
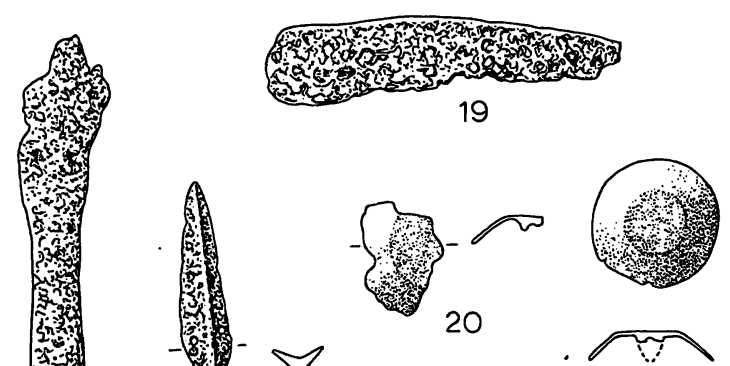

21
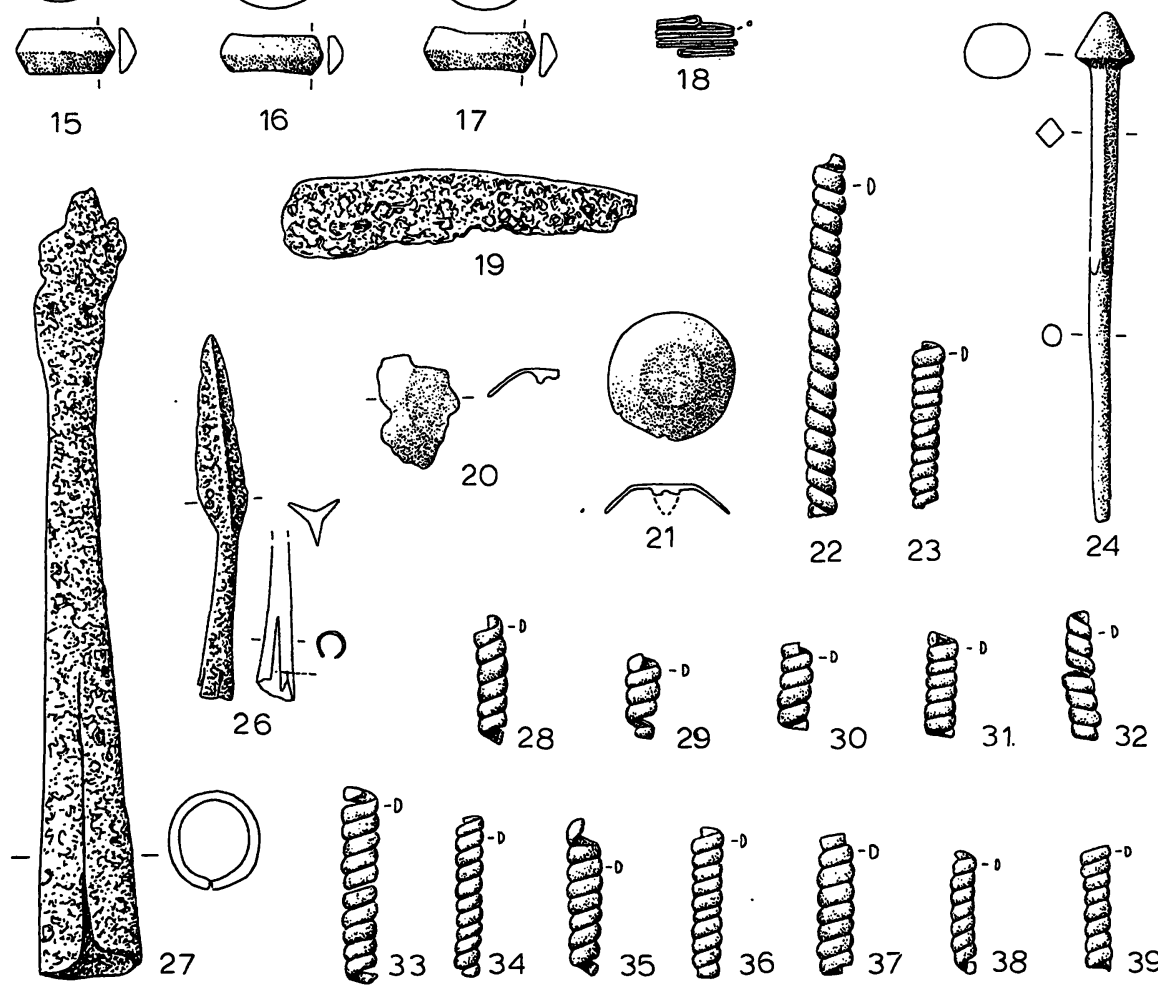

Abb. 18. Vergina. Feld Malamas, Grabhügel B. 1 Grab IV; 4-5 Grab VII; 20 Streufund. - Feld Malamas, Grabhügel $\Gamma$. 2-3 Grab IX; 6 Grab V; 7-8 Grab X; 9-19.21-27 Streufunde. - Aus einem der 1970 untersuchten Grabhügel. 28-39 keinem Grab zuweisbar. - 1-3.19.26-27 Eisen; 4-7.9-12.14-17.20-25.28-39 Bronze; 8 Fayence (?); 13.18 Gold. - M.1:2 


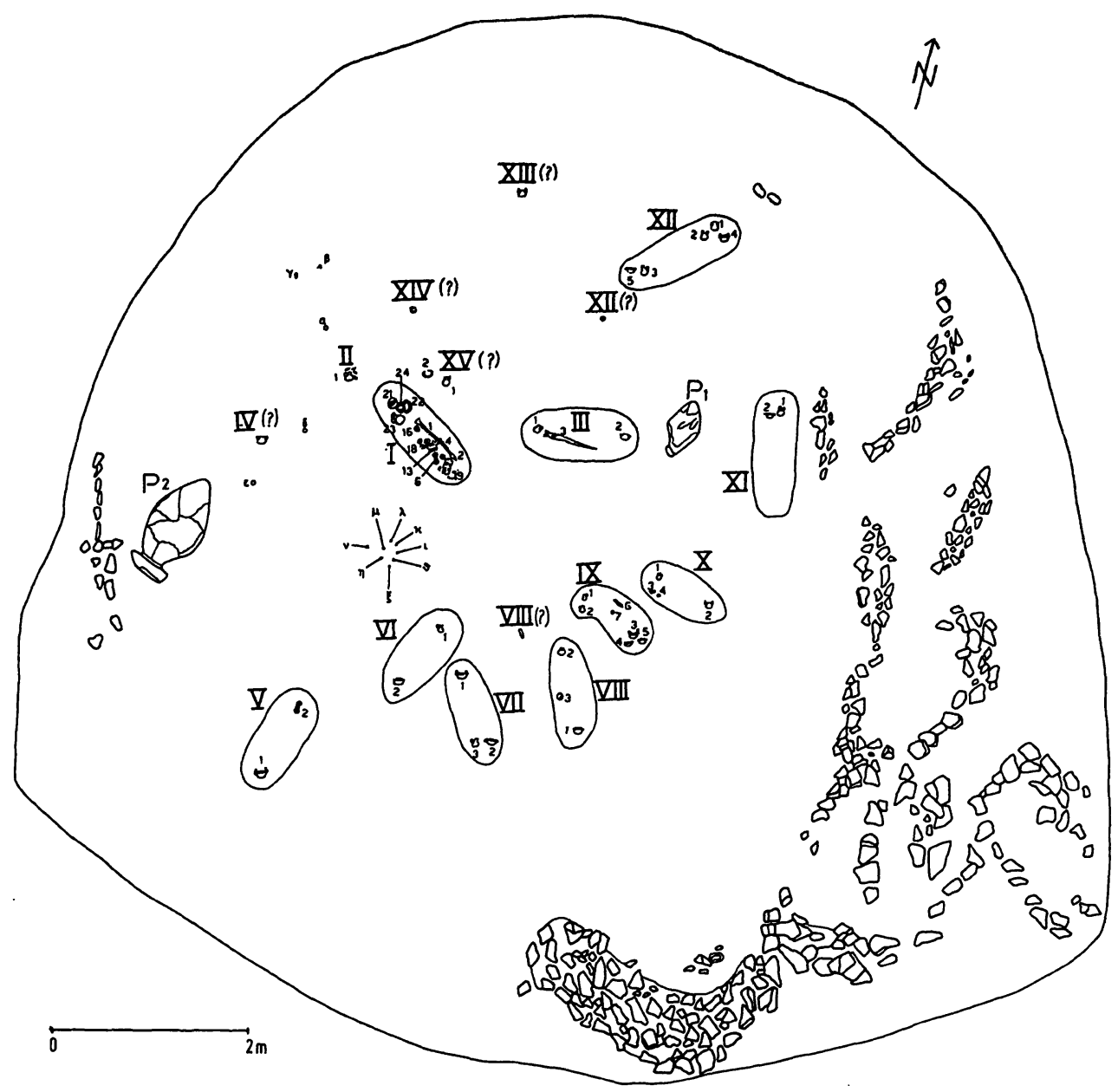

Abb. 19. Vergina. Feld Malamas, Grabhügel $\Gamma$

ring aus kräftigem Golddraht fand sich aber auch im Grab AГ $X$, das bereits in die Stufe II datiert wird.

In Makedonien ebenso wie im übrigen Griechenland waren die Lockenringe das nahezu einzige Schmuckstück, das während der frühen Eisenzeit, d.h. in submykenischer bis protogeometrischer Zeit, aus Gold gefertigt wurde ${ }^{78}$. Daß solche, durch ihr Material besonders kostbare Lockenringe in Vergina regelmäßiger Bestandteil derjenigen Grabausstattungen sind, die sich durch eine Anhäufung von Schmuck auszeichnen ${ }^{79}$, bedeutet keine Überraschung (Abb. 36-38). Sie können aber ebenso in

78 Kübler 1954, 183 f.; R. Higgins, Greek and Roman Jewellery ${ }^{2}(1980) 88$ ff.; Lefkandi I, 217 II.

79 Insbesondere die Gräber $\mathrm{AE} V, \Phi I I I, \mathrm{~A} \Delta I, \mathrm{AH} I I$, Malamas $\Gamma I, \mathrm{AZ} V I I, \mathrm{Y} I, \mathrm{LXV} \Gamma$. durchschnittlichen und selbst in bescheidenen Inventaren vorkommen. Wenn im Gegensatz dazu die bronzenen Lockenringe nie in besonders reichen Gräbern begegnen, sondern nur zusammen mit wenig aufwendigen Beigaben, dann zeichnet sich damit wohl eine unterschiedliche Wertigkeit ab.

Ebenfalls aus Gold oder aus Bronze sind in Vergina feine Drahtringchen gefertigt, bei denen das eine Ende zu einem spitzovalen Blech ausgehämmert und mit getriebenen Buckeln verziert ist (Abb. 17,18-19) ${ }^{80}$. Sie werden

80 Goldene Ringe aus den Gräbern AГ Pithos 3; III $\Delta$; Malamas B VI und aus einem zerstörten Grabhügel: K. Rhomiopoulou, Arch. Ephemeris 1969 Chronika 13 f. Abb.2-3. 


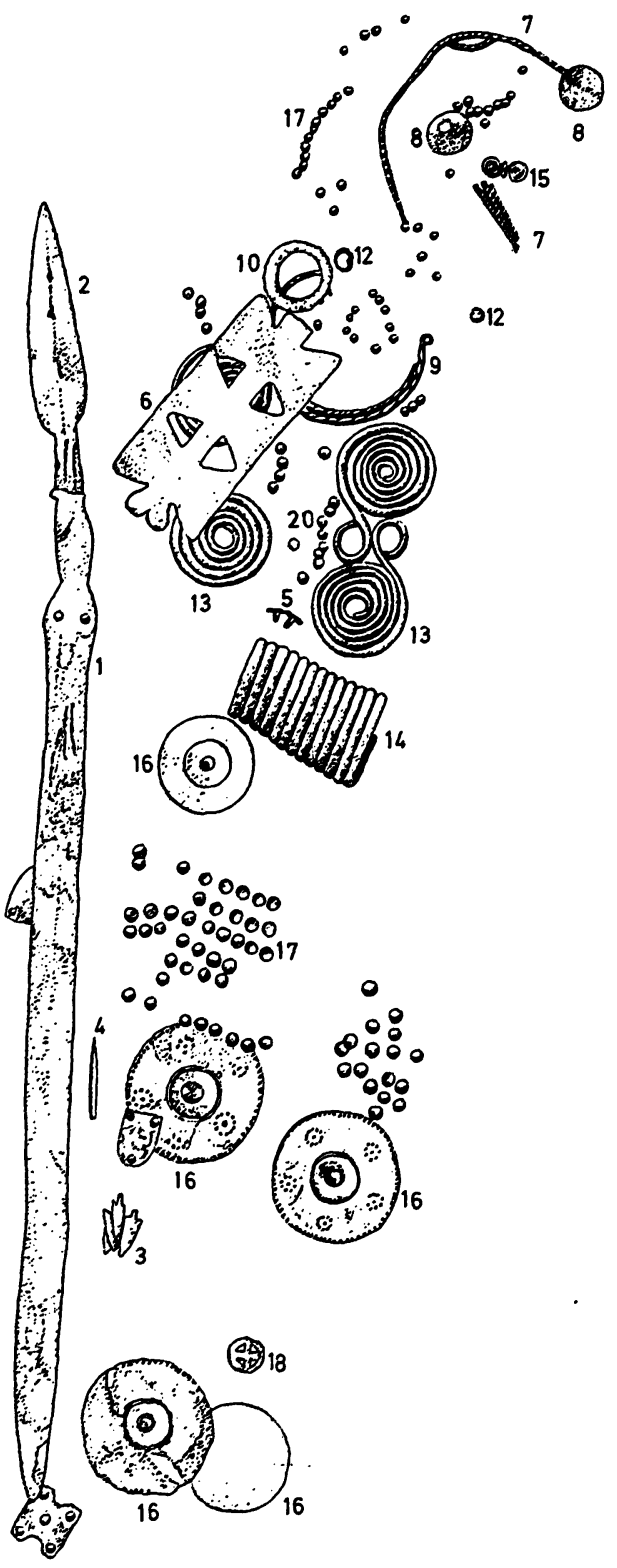

Abb. 20. Vergina. Feld Malamas, Grabhügel $\Gamma$. Grab I

Abb. 22. Vergina. Feld Malamas, Grabhügel r. Grab I (Teilansicht)

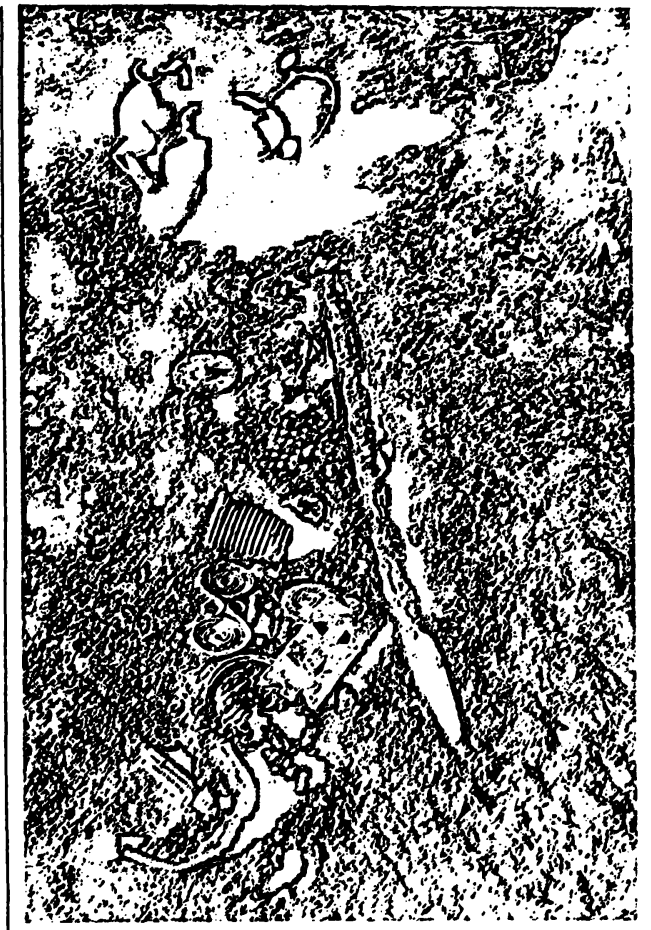

Abb.21. Vergina. Feld Malamas, Grabhügel $\Gamma$. Grab I

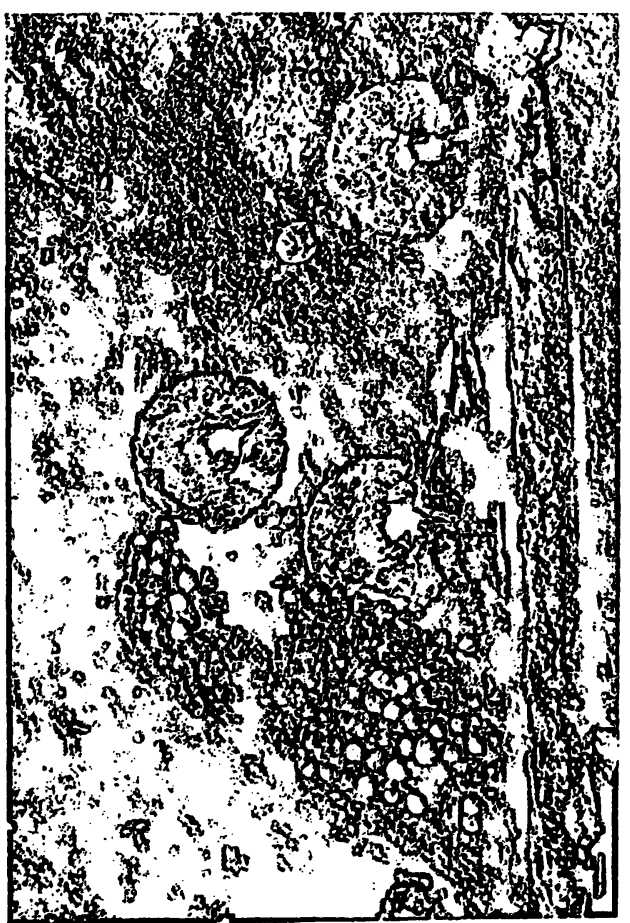




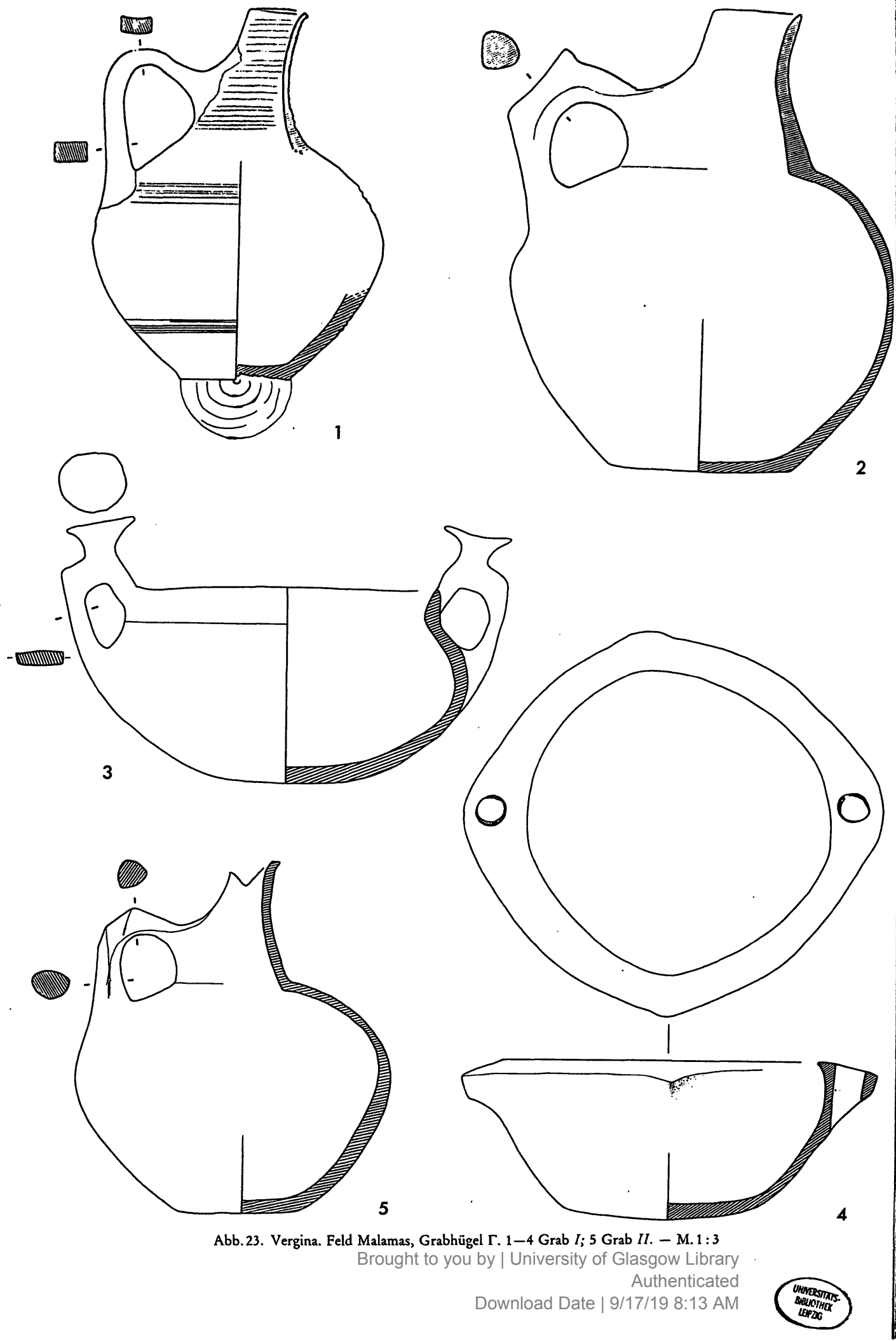




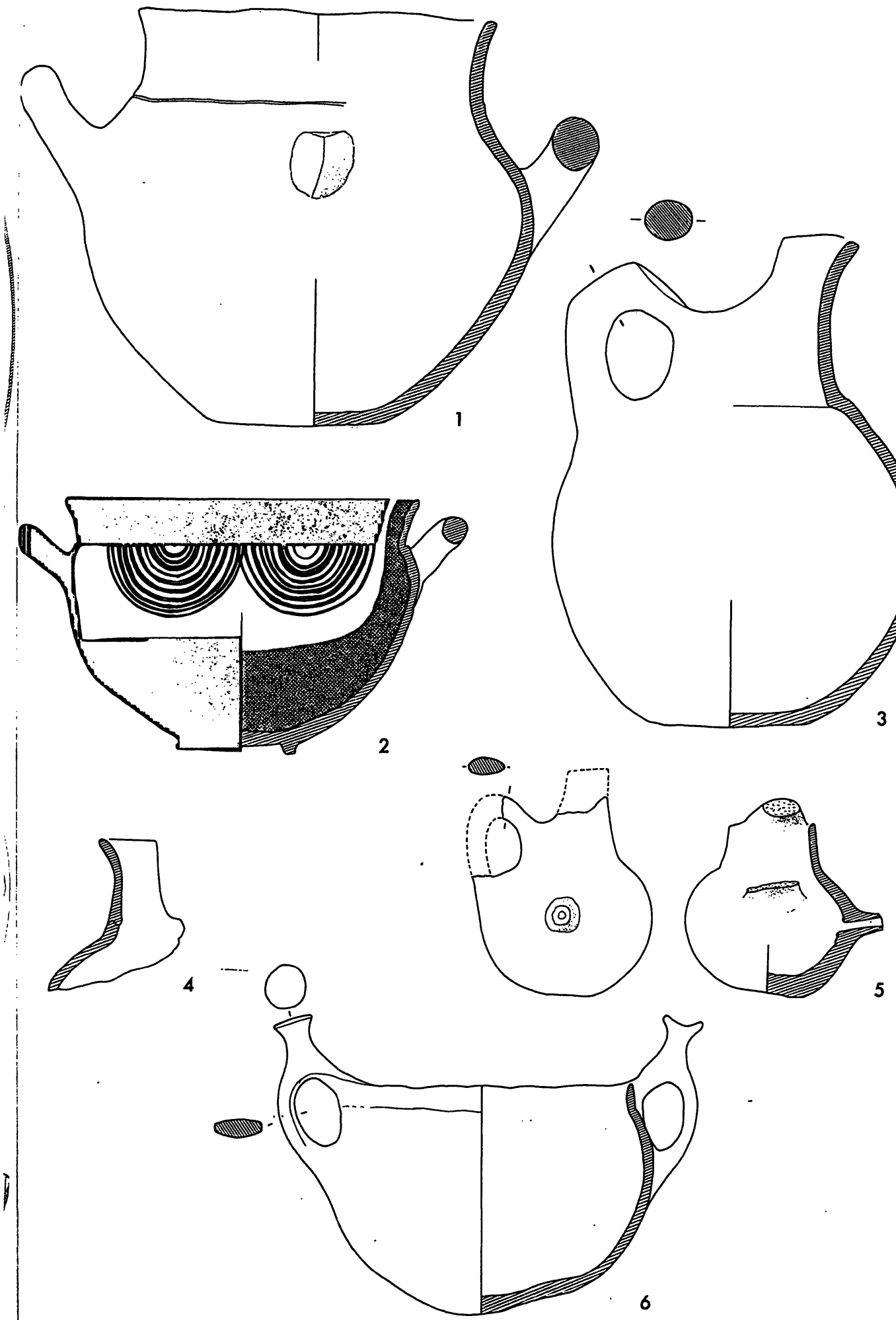

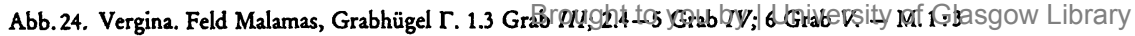




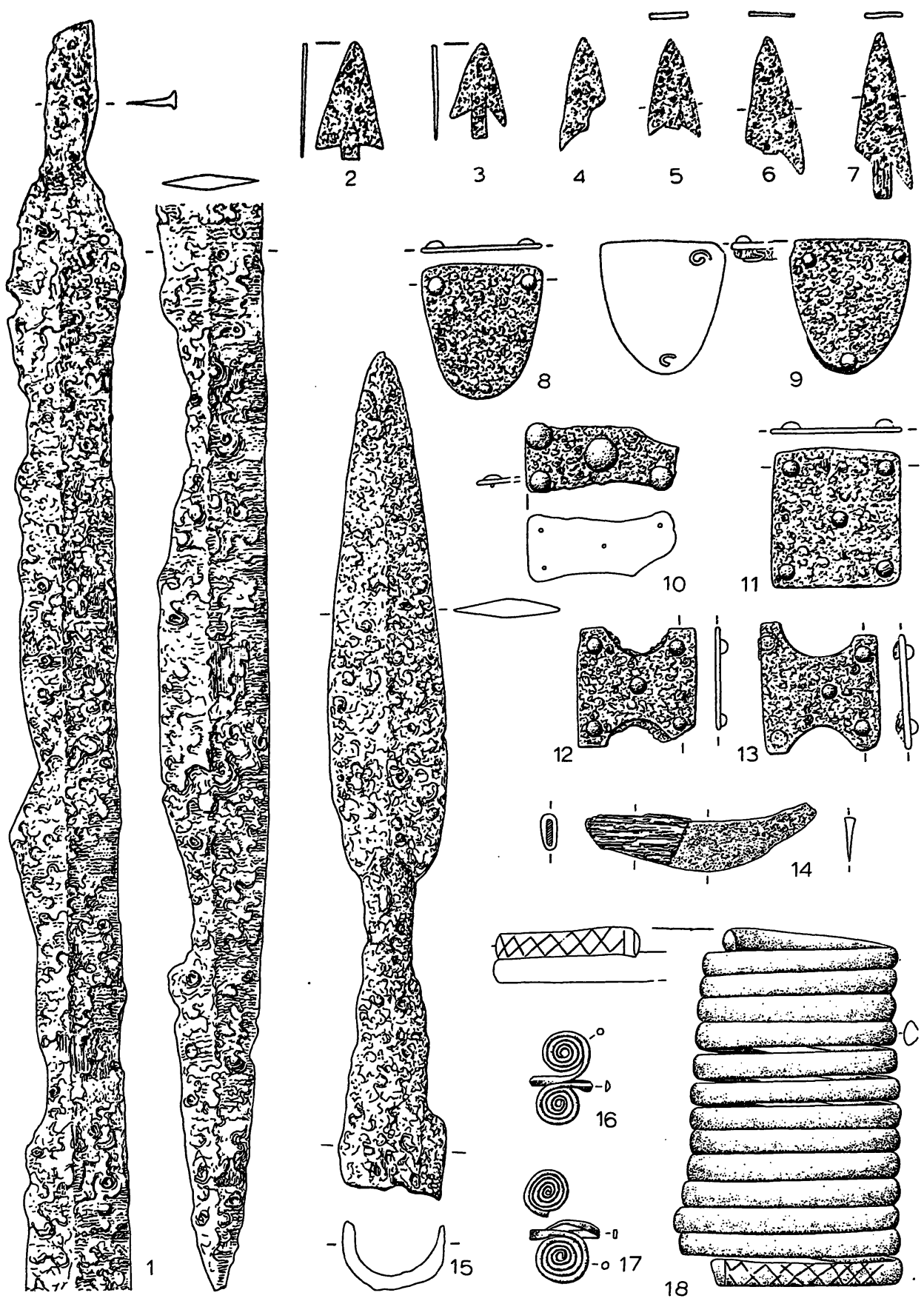

Abb. 25. Vergina. Feld Malamas, Grabhügel Г. 1-18 Grab I. - 1-7.14-15 Eisen; 8-13 Eisen und Bronze; 16-18 Bronze. M. $1: 2$ 


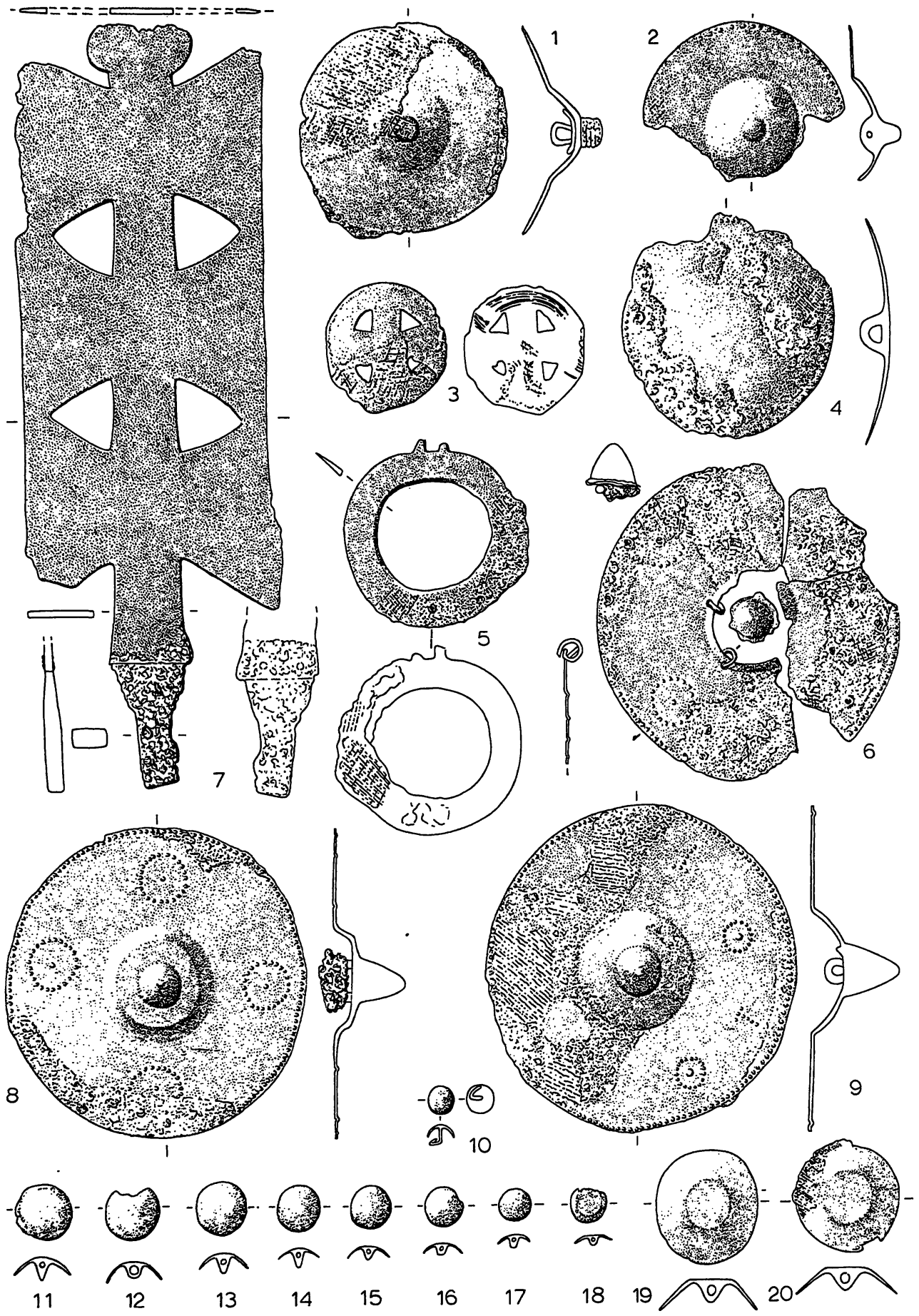

Abb. 26. Vergina. Feld Malamas, Grabhügel Г. 1-20 Grab I. - 1-6.8-20 Bronze; 7 Bronze und Eisen. - M. $1: 2$ 


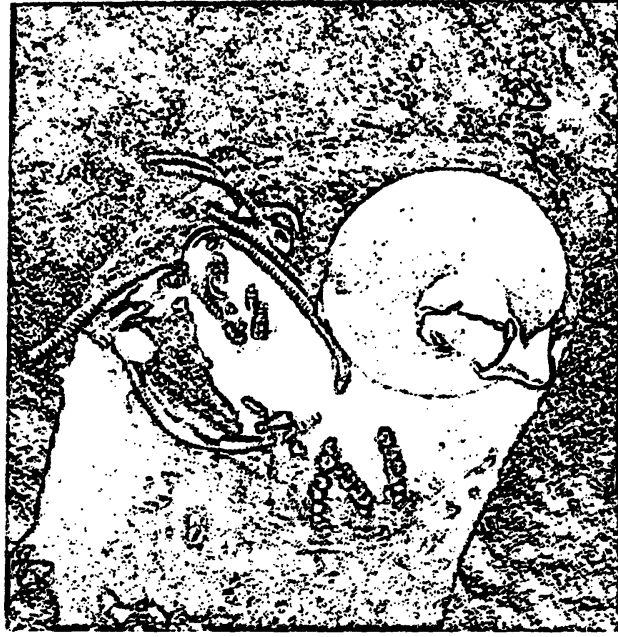

Abb. 27. Vergina. Feld Malamas, Grabhügel $\Gamma$. Grab II

meist als Ohrringe bezeichnet ${ }^{81}$. Da sie ebenso wie die goldenen Spiralringe in den Gräbern paarweise auftreten, in der Nähe des Schädels liegen und Durchmesser von $1,3-2 \mathrm{~cm}$ haben, könnte es sich um Lockenringe handeln und zwar um einen späten Typ, der in der Stufe IV der Nekropole die bisher üblichen Spiralen ersetzt ${ }^{82}$.

\section{Halsringe}

Bei den offenen, tordierten Halsringen von Vergina sind die glatten Enden von rhombischem oder spitzovalem Querschnitt bandförmig ausgehämmert und nach außen zu einer Spirale eingerollt ${ }^{83}$. In diesen Merkmalen unterscheiden sich die makedonischen Halsringe von thessalischen oder balkanischen Ausführungen ${ }^{84}$. In den Gräbern von Vergina sind Halsringe keine sehr häufige Beigabe ${ }^{85}$. Sie scheinen nicht Bestandteil einer regelhaft wiederholten Grabausstattung - d.h. Tracht - zu sein. Sie begegnen vielmehr in vermutlichen Kindergräbern mit bescheidenen Beigaben ${ }^{86}$ ebenso wie in vermutlichen

81 I. Mikulčić, Pelagonija u svetlosti arheoloških nalaza (1966) 39; Rhomiopoulou (Anm. 80) 13 f.; Andronikos 1969, 259.

82 Die Gräber AT Pithos 3 und III $\Delta$ sind in die Phase IVA datiert. Den späten Ansatz solcher Goldringe bestätigt auch der Grabfund von Petilep in Pelagonien: Mikulčić (Anm. 81) 38 f. Taf. 12,21.

83 An einigen Exemplaren der Grabung Andronikos ist nur eines der eingerollten Enden erhalten; einen Haken-Ösenverschluß gibt es an den Halsringen von Vergina nicht. Vgl. dagegen Andronikos 1969, $247 \mathrm{f}$.

84 J.Bouzek, Graeco-Macedonian Bronzes (1973) 97; Kilian 1975 b, 171 .

${ }^{85}$ Es sind insgesamt 15 Exemplare aus 11 Gräbern und ein Streufund aus Malamas Hügel $\Gamma$.

8h z. B. A VI, Malamas B II.

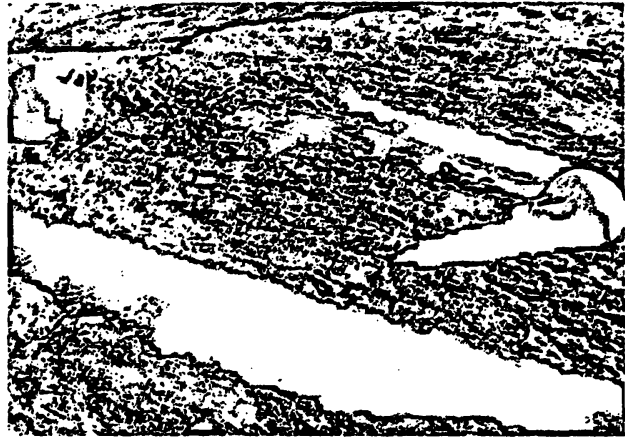

Abb. 28. Vergina. Feld Malamas, Grabhügel $\Gamma$. Grab III

Frauengräbern zusammen mit besonders reichem oder auch ärmlichem Trachtzubehö $r^{87}$. In drei ungestörten Gräbern ist zudem das Tragen von zwei gleichartigen Halsringen bezeugt ${ }^{88}$. Durch die kleine Bogenfibel mit symmetrischem Nadelhalter wird der Halsring mit echter Torsion aus Grab A $\Gamma$ VI in die Stufe II der Nekropole datiert ${ }^{89}$. Die Exemplare der Phasen III A-C haben Scheintorsion. Das Fehlen von Halsringen in den späten Gräbern der Stufe IV ist wohl zufällig, wie Funde des 7. Jhs. aus Axiokastron vermuten lassen ${ }^{90}$.

Perlen

Im Gegensatz zum südlichen Griechenland, wo bunte Glasperlen als Beigaben in Gräbern und als Votive in Heiligtümern ganz üblich sind"1, begegnen sie im früheisenzeitlichen Makedonien recht selten". Aus Vergina waren bisher nur drei Exemplare der Grabung Andronikos bekannt ${ }^{93}$. Nach der Beschreibung wäre die Perle $\delta 1$ aus Grab AZ VII mit der Perle (3) aus der Fundgruppe $V I b$ im Hügel B (Feld Malamas) zu vergleichen ${ }^{9 t}$. Die Befunde in den Gräbern $\mathrm{O} I, \mathrm{~A} \triangle I, \mathrm{AZIV}$ und $\mathrm{AZVII}$ lassen vermuten, daß die Glasperlen zusammen mit anderen Perlen auf eine Halskette aufgezogen waren ${ }^{95}$. Die Mehrzahl aller Perlen aus den Gräbern von Vergina besteht aus einem rötlichen Stein, der als Sard (Sardonyx)

87 z. B. die reichen Gräber N $I V$, AZ VII, LXV $\Xi$, Malamas $\Gamma I$ und die armen Gräber $N I I, L X V B A$.

${ }^{88}$ Gräber LXV $\Pi, \mathrm{LXV} B \Gamma$, Malamas $\Gamma I$

89 So Kilian 1975 a, 66.

D Petsas, Arch. Deltion 17, 1961-62 Chronika 214 Taf. 253,a.

9 Vgl. Perachora II: T.J. Dunbabin, Pottery, Ivories, Scarabs and other Objects from the Votive Deposits of Hera Limenia (1962) 519 f.; Kilian 1975 b, Taf.78,1-59; Lefkandi I, 84 f.; 223.

92 A. Hochstetter, Kastanas/Die Kleinfunde. Prähist. Arch. Südosteuropa 6 (1987) $93 \mathrm{f}$.

93 Andronikos 1969, 254 f. (Gräber O I, A $\triangle I$, AZ VII).

94 Ebd. 143.

95 Möglicherweise als Mittelperle, wie Andronikos ebd. 255 annimmt. 
bestimmt wurde\%. Die Anzahl von 56 Perlen in Grab Malamas $\Gamma I$ ist für Vergina groß"7; ohne Abstände auf eine Schnur aufgezogen ergäben die Bergkristallperle, die Scheibenperle aus Fayence (?) und die 54 Sardperlen eine kurze, am Hals anliegende Kette. Die regelmäßig in ungestörten Bestattungen beobachtete Fundlage von nur einigen wenigen Perlen im Bereich des $\mathrm{Halses}$, manchmal noch in der ursprünglichen Reihung ${ }^{98}$, bedeutet entweder, daß bei einer Perlenkette aus vorwiegend vergänglichem Material nur die Mitte von den kostbaren Steinperlen besetzt war, oder daß diese allein auf eine Schnur aufgezogen am Hals getragen wurden. Bei einer derartigen Tragweise käme den Sard- und Glasperlen nicht so sehr Schmuckfunktion als Amulettcharakter zu. In die gleiche Richtung weisen auch die vereinzelten Sardperlen, die mehrfach in vermutlichen Kindergräbern begegnen". Am Hals getragene Perlen aus Sard, seltener anderen Halbedelsteinen, Fayence (?), Ton und Glas gehören zu den üblichen Beigaben in Frauengräbern, wobei ganz offensichtlich ihre $\mathrm{Zahl}$ in den Bestattungen mit reichem Trachtzubehör größer ist als in den bescheidenen Inven$\operatorname{taren}^{100}$. Zeitlich scheinen sie weitgehend auf die Stufe III der Nekropole begrenzt zu sein. Deutlich wird das vor allem an den Trachten mit reichem Kopfschmuck, zu denen während der Phasen III A-C stets mehrere Sardperlen gehören, während die Gräber der Stufe IV mit Diadem keinerlei Perlen enthalten (Abb. 36). In Gräbern mit Waffen oder Eisenmesser kommen ab der Phase IV A gelegentlich eine bis drei Bronze- oder Bleiperlen vor ${ }^{101}$. Nach ihrer Fundlage scheinen sie nicht am Hals getragen worden zu sein.

Fibeln

An Fibeln liegen aus den vier Grabhügeln der Felder Lazaridis und Malamas nur Brillenfibeln vor. Sie gehören alle dem in Vergina zahlreich vertretenen Typ mit mittelgroßer Achterschleife an ${ }^{102}$. Ihre Größe reicht von $3-19,5 \mathrm{~cm}$. Die kleinformatigen Exemplare bestehen

\% Ebd. 254. Ebenso aus Hügelgräbern am Fuß des Olymp: Ancient Macedonia [Ausstellungskatalog Sidney] (1988) 169 Nr. 86.

97 Die Gräber $\mathrm{A} \triangle I, \mathrm{AZ} V I I$ und $\mathrm{LXV} A P$ enthalten mit $47 \mathrm{bzw}$. 42 Perlen bereits merklich weniger.

98 Gräber A $I X, \Delta V, \mathrm{E} I, \mathrm{E} V I, \mathrm{~K} V, \mathrm{~N} I I I, \mathrm{~N} V I, \mathrm{~N} V I I, \mathrm{~N} I X$, $\mathrm{N} X, \mathrm{~N}$ Pithos $1, \mathrm{O} I, \mathrm{Y} I, \Phi I, \Phi I I I, \Phi V I I I, \Phi$ Pithos 2, $\mathrm{AA} V, \mathrm{AB} I, \mathrm{AB}$ Pithos 4, $\mathrm{A} \Gamma I I I, \mathrm{~A} \Gamma V, \mathrm{~A} \Gamma V I I I, \mathrm{~A} \Gamma X$, $\mathrm{AE} I I, \mathrm{AE} I I I, \mathrm{AE} V I I, \mathrm{AE} I X, \mathrm{AZI}, \mathrm{AZIII}, \mathrm{AZ} X, \mathrm{AH} V$, AH VI. Gleiche Beobachtung in der Nekropole von Vitsa, Epirus: Vokotopoulou 1986, $315 \mathrm{ff}$. Entsprechung zu der Perle aus schwarzer Glaspaste mit runden Einlagen (Nr.3): ebd. Zeichnung 110a (Grab 113).

9 z. B. Gräber NVI, AZI, AHV, AHIX, Malamas B II.

100 Die Zahlen liegen bei etwa 1-6 Perlen in bescheidenen Inventaren und $13-56$ in den reichen.

101 Gräber III $K, \operatorname{LXIV} \Gamma, \operatorname{LXV} P, \operatorname{LXV} A \Gamma, \operatorname{LXVIII} \Delta$, LXVIII $E$, LXVIII Z, AZIII.

102 Alexander Typ Ib (J.Alexander, Am. Journal Arch. 69, 1965, $8 \mathrm{f}$ ); siehe auch Andronikos 1969, $227 \mathrm{ff}$;; Kilian 1975 b, 145 (Typ BI). häufig in allen Teilen aus rundstabigem Draht, bei den großen Fibeln hat der Draht für Achterschleife und erste Windung der Spiralscheiben rhombischen Querschnitt ${ }^{103}$. Einzige Ausnahme ist die große Brillenfibel Nr.898 (Abb. 18,14), die ganz aus rundstabigem Draht besteht. Wie bereits Andronikos in dem von ihm gegrabenen Nekropolenabschnitt festgestellt hat, werden die Brillenfibeln häufig als Paar auf der rechten und linken Schulter getragen $^{104}$. Es fehlt jedoch nicht an ungestörten Bestattungen mit nur einer Brillenfibel. Auffällig ist dabei, daß kleine Brillenfibeln häufiger einzeln vorkommen als große $^{105}$, während die Paare wesentlich öfter von großen Exemplaren gebildet sind als von kleinen ${ }^{106}$. Andronikos sah in der Fibelgröße einen Indikator für das Alter der Bestatteten ${ }^{107}$. Die Verteilung großer und kleiner Brillenfibeln auf die Beigabenkombinationen würde unter dieser Prämisse ergeben, daß allein die besonders reiche Tracht mit Kopf-, Hals- und Armschmuck, Gürtel, Faleren oder Drillingsbeilen den erwachsenen Frauen vorbehalten war, während alle übrigen Ausstattungen mit Brillenfibeln nicht altersspezifisch sind, da sie gleicherweise große wie kleine Fibeln enthalten können. Eine funktionsbezogene Unterscheidung von kleinen Fibeln am Kopf-Schultertuch und großen Fibeln am Gewand ${ }^{108}$ kann ebenfalls nicht als Regel gelten, da auch kleinformatige Brillenfibeln in Schulterposition begegnen ${ }^{109}$.

Brillenfibeln setzen in Vergina mit der Phase III A ein. Die von Kilian als späte Variante der Stufe IV herausgestellte Ausführung mit schuppenartiger Wickelung des vierkantigen Drahts ${ }^{110}$ ist in den hier vorgestellten Hügeln nicht vertreten.

Nadeln

Die zwei Bronzenadeln aus Malamas Hügel $\Gamma$ gehören mit ihrem großen Kegelkopf und dem im oberen Teil

${ }^{103}$ Als Grenzwerte für kleine Fibeln lassen sich etwa $3-8 \mathrm{~cm}$ bestimmen, für große $9,7-19,3 \mathrm{~cm}$.

10t Andronikos 1969, 227.

105 Gräber mit nur einer kleinen Brillenfibel: $\triangle I I I, Z \mathrm{~V}, \mathrm{Z}$ Pithos 2, N $I I, \mathrm{~N} X I I, \mathrm{~A} \Gamma X V I I, \mathrm{~A} \Gamma$ Pithos 3, AE $I X, \mathrm{AZI}, \mathrm{LXV} \Pi$, LXV $\Phi$, LXV $B A$, LXV $B E$. - Gräber mit nur einer großen Brillenfibel: $\mathrm{A} I X, \Xi I V, \mathrm{AA} V$ (gestört?), $\mathrm{AB} I, \mathrm{AB}$ Pithos 3, LXV $A \Xi$ (?), Hügel Lazaridis $I V$, Malamas $\Gamma$ Pithos 1.

106 Gräber mit zwei großen Brillenfibeln: $\mathrm{E} I, \mathrm{~K} V, \mathrm{~N} I V, \mathrm{~N} I X$, $\mathrm{O} I, \mathrm{~T} V, \mathrm{Y} I, \mathrm{Y} I I I, \Phi I, \Phi I I I, \mathrm{AA} I V, \mathrm{AE} I I I, \mathrm{AE} V$, AEVII, A $\Delta I, \mathrm{AZ} V I I, \mathrm{AH} I I, \mathrm{III} \Delta, \mathrm{III} \theta, \mathrm{LXV} \Gamma, \mathrm{LXV} I$, LXV AO, Malamas $\Gamma I$. - Gräber mit zwei kleinen Brillenfibeln: $Z I, Z I V, Z V I, \mathrm{~N} I I I, \mathrm{AB}$ Pithos $1, \mathrm{~A} \Gamma V, \mathrm{~A} \Gamma X X$, $\mathrm{AZ} X, \operatorname{III} I, \operatorname{III} A, \operatorname{LXV} K, \operatorname{LXV} A B, \operatorname{LXV} B \Theta$.

107 Andronikos 1969, 227: kleine Fibeln bei 1 lädchen, große bei Frauen.

${ }_{108}$ Sicher belegt in den Gräbern Malamas $\Gamma I, \mathrm{AZ} V I I, \mathrm{~N} I V$, LXV B, , A $X X$.

109 Befunde in den Gräbern ZIV, NIII, AГV, АГXX.

110 Kilian 1975 a, 72. Das chronologisch wichtige Merkmal ist die Wickeltechnik, mit der eine glatte Oberfläche der Spiralscheibe erreicht wird. Brillenfibeln, die in allen Teilen aus vierkantigem Draht bestehen, sind in Vitsa bereits ins 9.Jh. v. Chr. datiert: Vokotopoulou 1986, 309 f. 


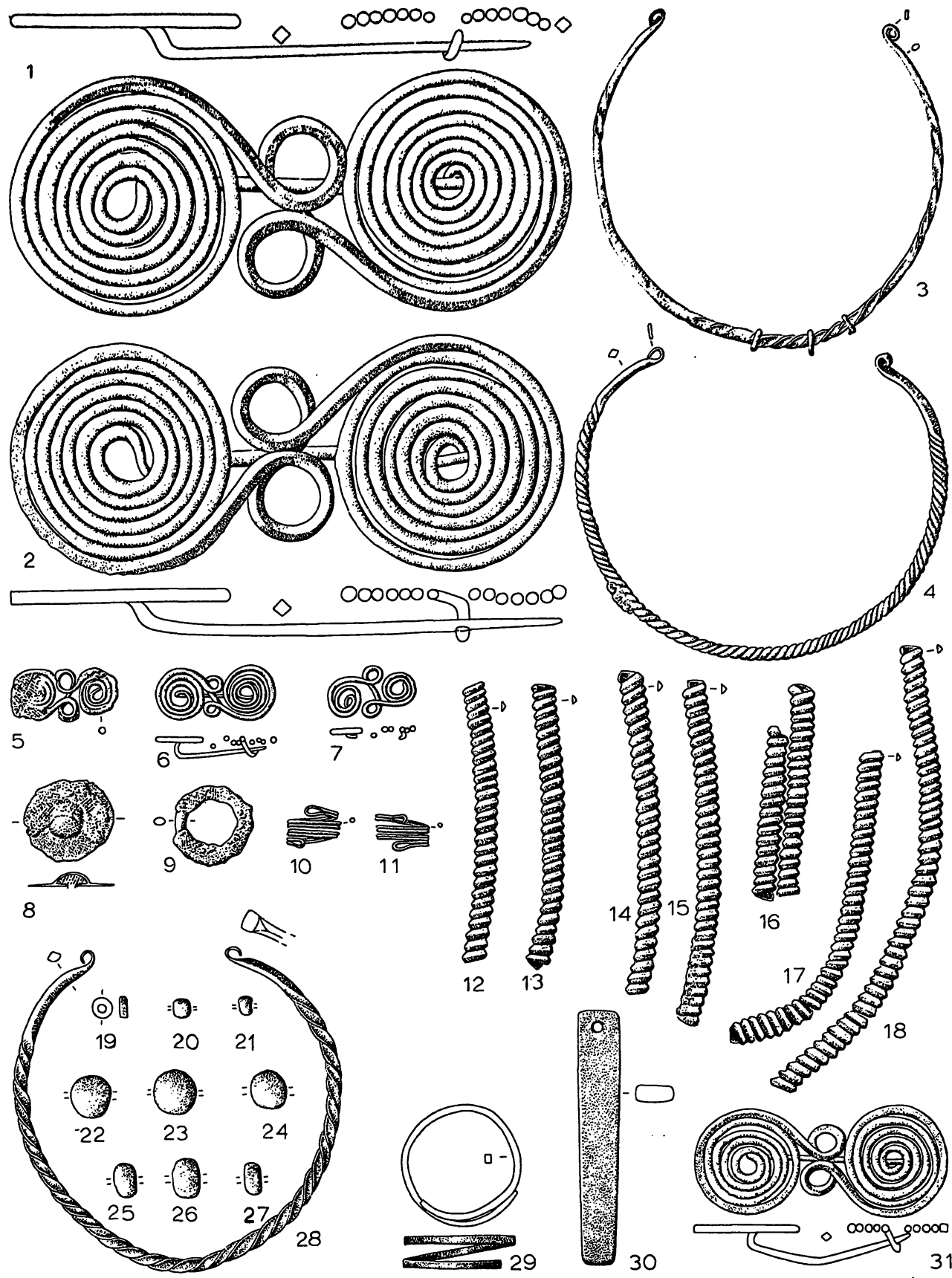

Abb. 29. V̀ergina. Feld Malamas, Grabhügel Г. 1-27 Grab I; 28 Grab XV; 29 Grab VIII; 31 Pithos 1; 30 Einzelfund zwischen Gräbern VII und VIII. - 1-9.12-18.28-29.31 Bronze; 10-11 Gold; 19 Fayence (?); 20 Bergkristall; 21-27 Sard; 30 Stein. M. $1: 2$ 


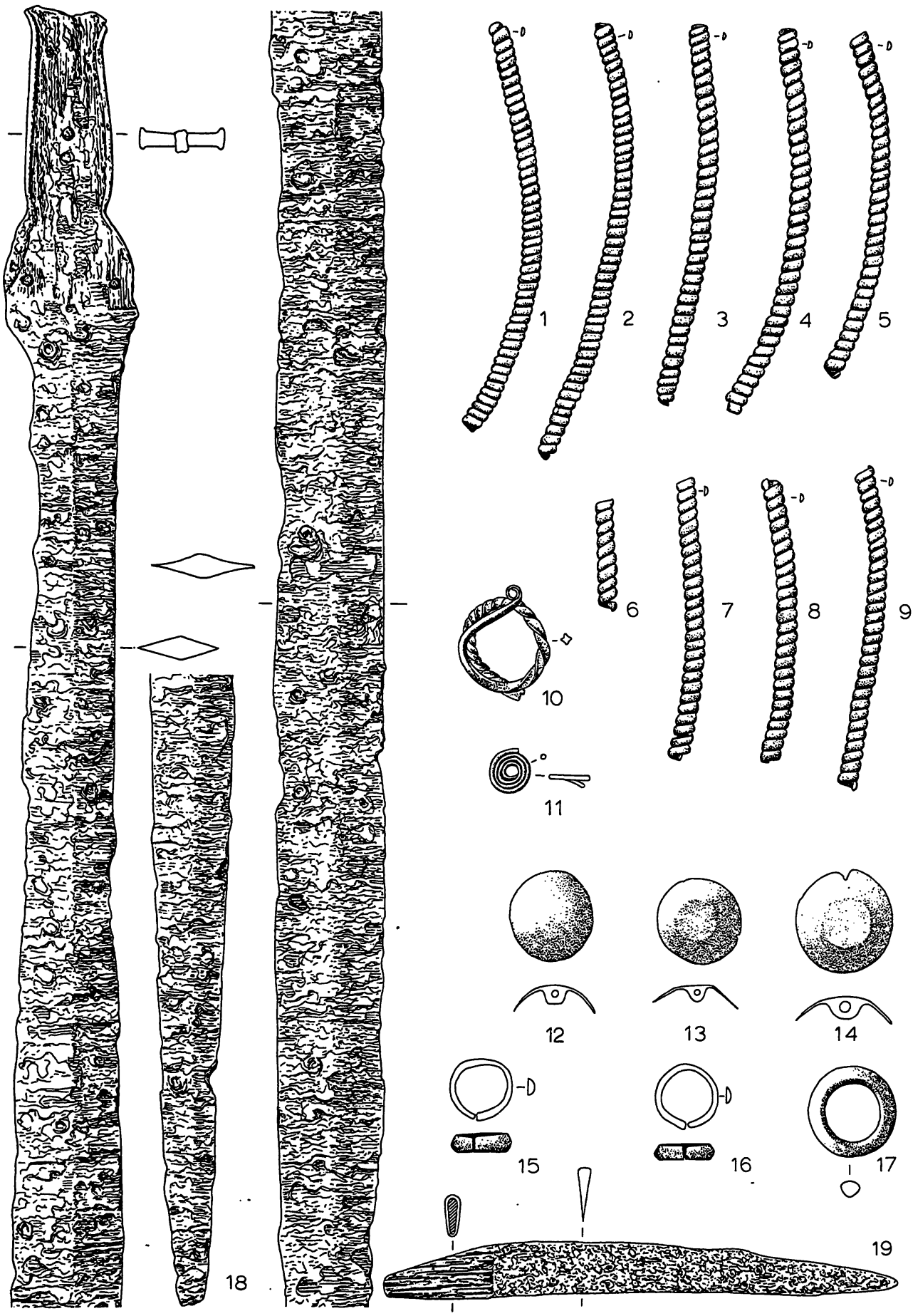

Abb. 30. Vergina. Feld Malamas, Grabhügel Г. 1-17 Grab II; 18-19 Grab III. - 1-17 Bronze; 18-19 Eisen. - M. 1:2 

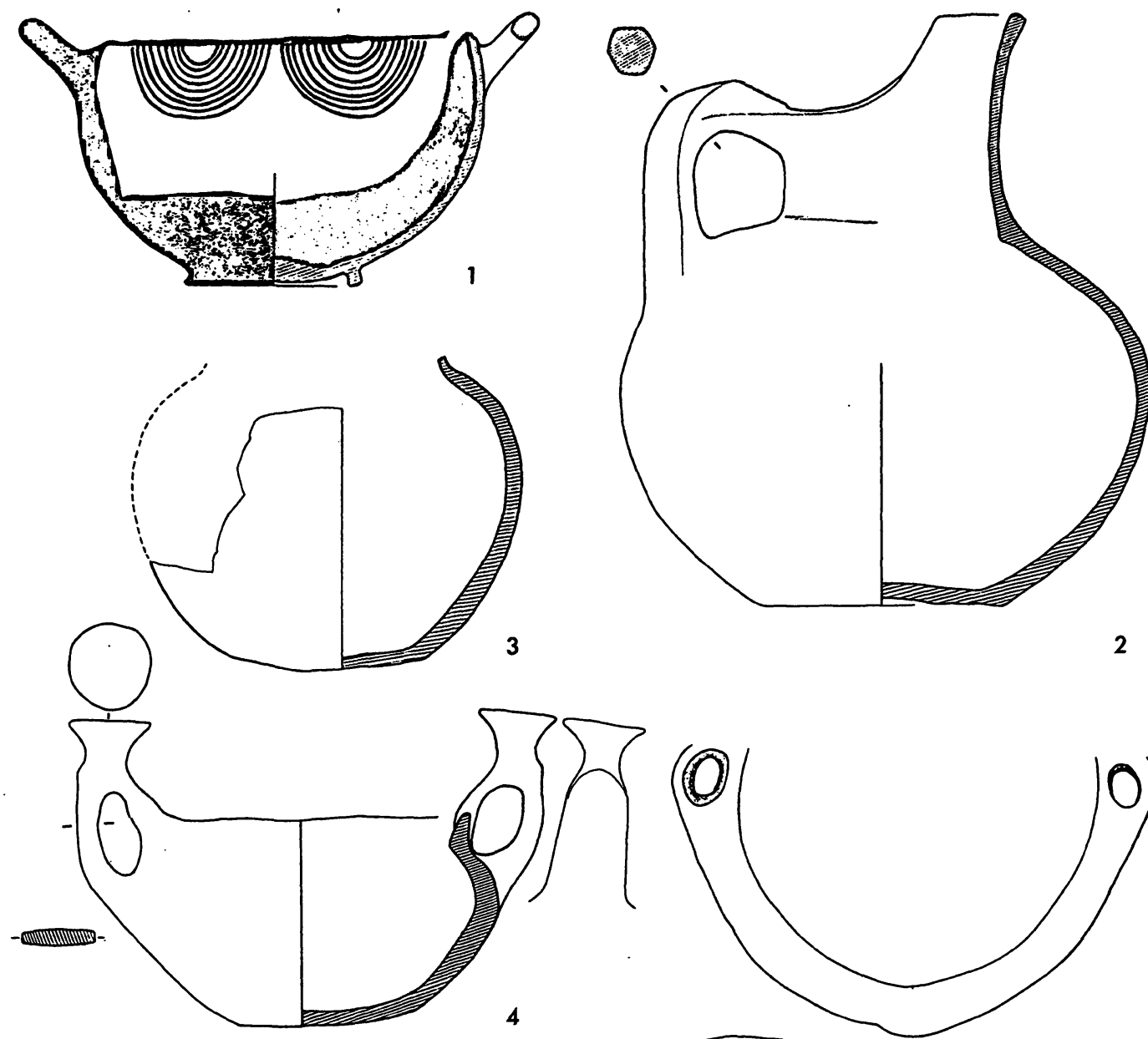

2

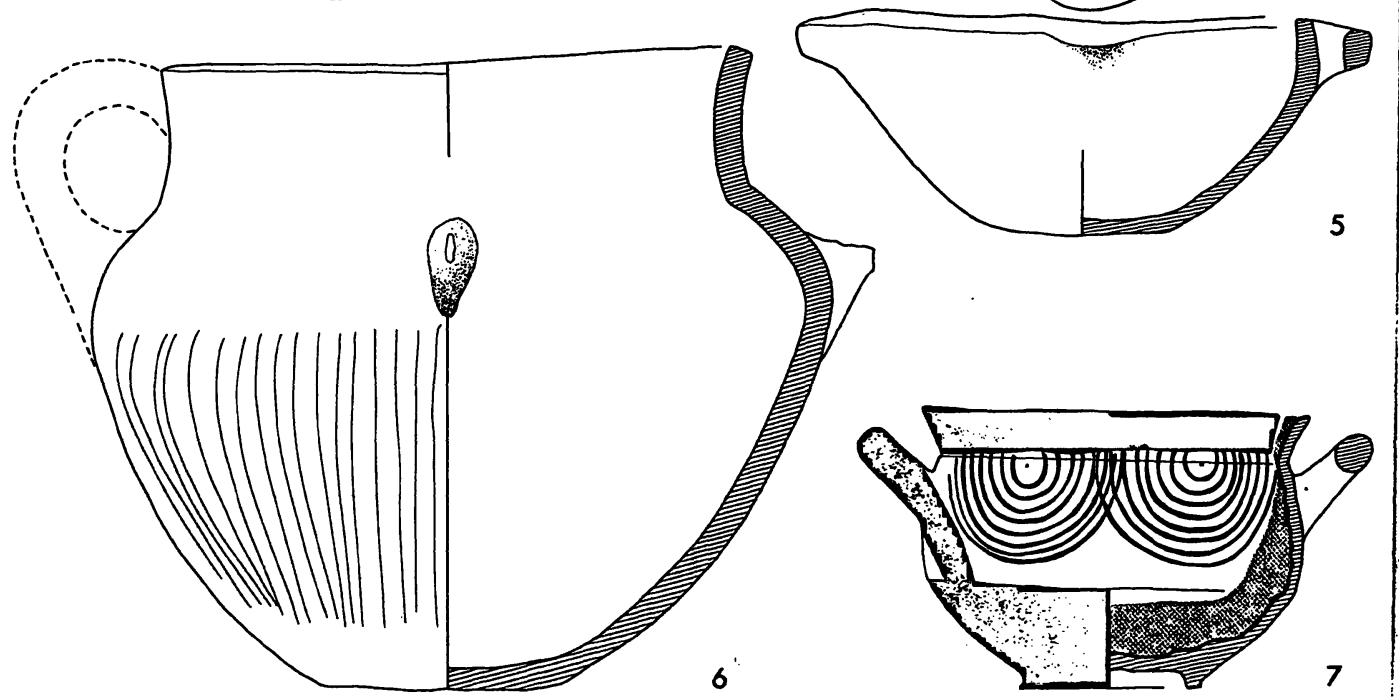

Abb. 31. Vergina. Feld Malamas, Grabhügel $\Gamma .1 .3$ Grab VI; 2.4-5 Grab VII; 6-7 Grab VIII. - M. $1: 3$

Brought to you by | University of Glasgow Library 

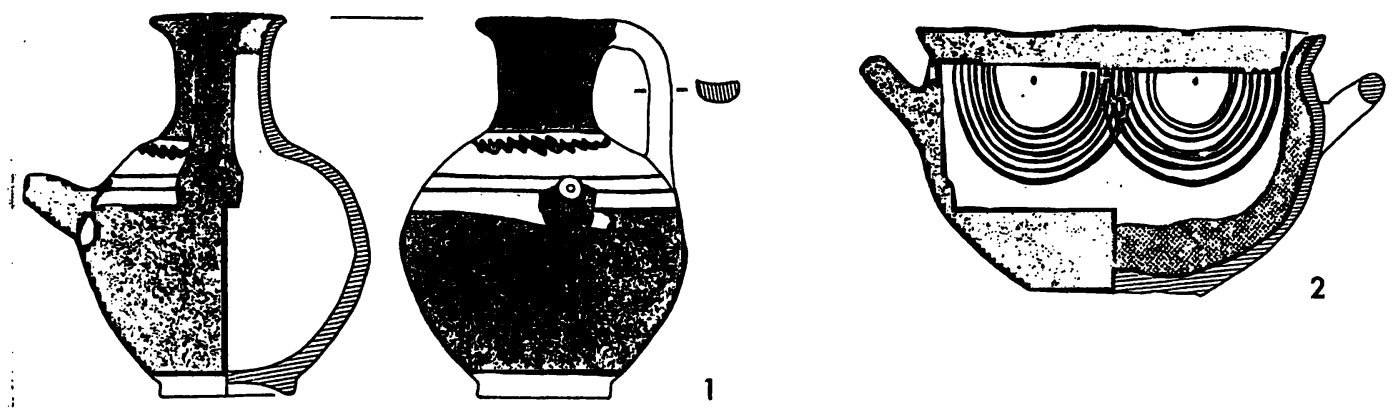

1

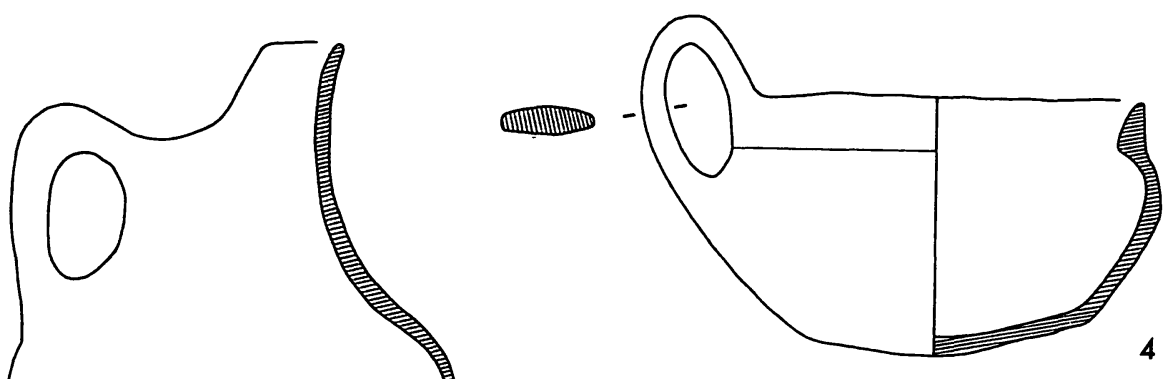

3

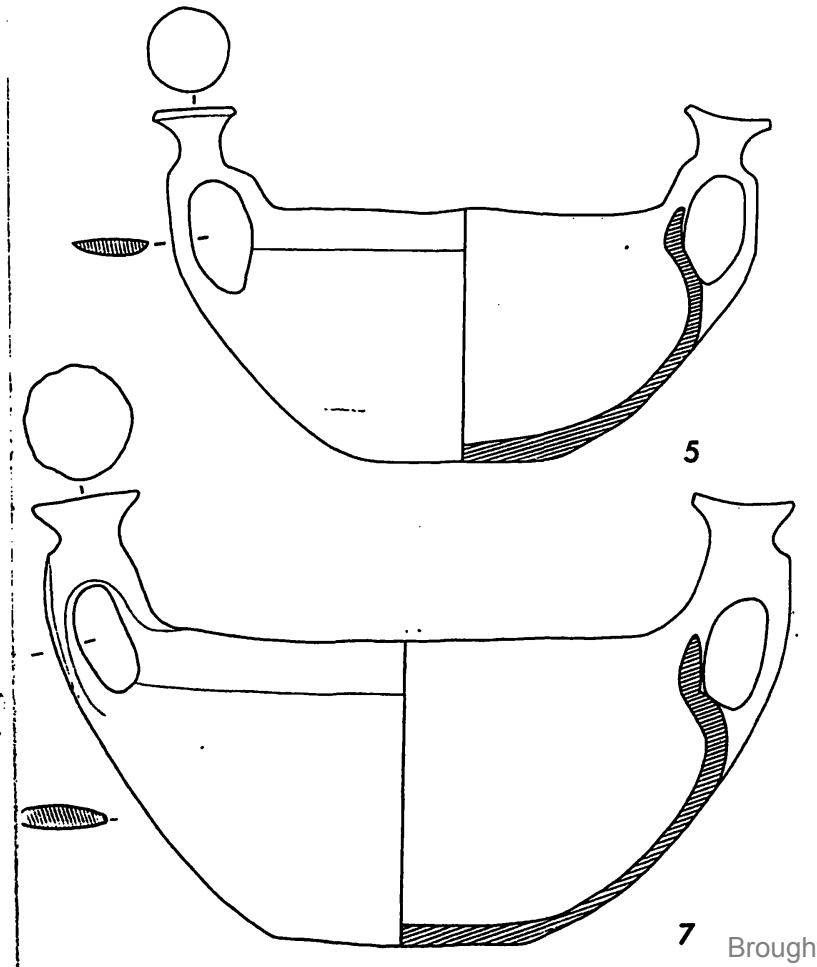

-

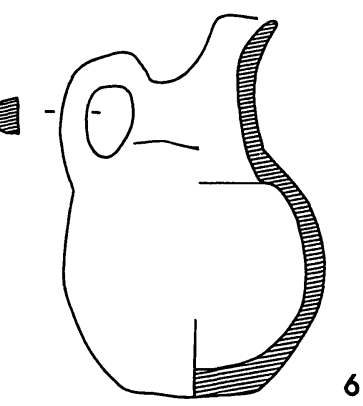

Abb. 32. Vergina. Feld Malamas, Grabhügel $\Gamma .1-5$ Grab IX; 6-7 Grab X; 8 Grab XI. - M. 1/3uthenticated 


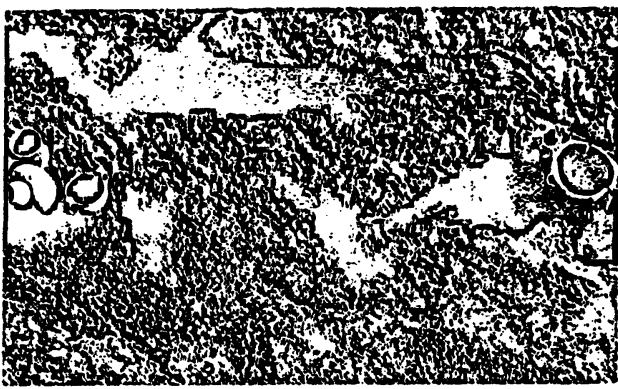

Abb.33. Vergina. Feld Malamas, Grabhügel $\Gamma$. Grab XII

vierkantigen Schaft (Abb. 18,24-25) einem Typ an, der in Vergina bisher nur mit einem Exemplar belegt war" ${ }^{\prime \prime}$. Die Datierung dieses Typs an das Ende der Stufe I und in den Beginn der Stufe II der Makedonischen Eisenzeit, d.h. in die Phasen III C-IV A von Vergina, ist durch mehrere Grabfunde von Chauchitsa gesichert ${ }^{112}$. Daraus ergibt sich für Vergina die merkwürdige Feststellung, daß zu Beginn der Nekropole in den Gräbern Paare langer Nadeln liegen; während der Phasen III A-B kommen nur Fibeln vor und $a b$ Phase III $C$ treten wieder lange Nadeln auf, jetzt allerdings einzeln ${ }^{113}$. Mit diesen Veränderungen der Grabausstattung zeichnet sich vermutlich das Aufkommen neuer, im Zuschnitt oder in der Tragweise andersartiger Gewänder ab.

\section{Armspiralen}

Die sechs Armspiralen aus den Hügeln $B$ und $\Gamma$ (beide Feld Malamas) haben den üblichen $D$-förmigen bis spitzovalen Querschnitt. Andronikos unterschied große Armspiralen mit 6-15 Windungen und kleine mit 4-5 Windungen ${ }^{114}$. Dies scheint keine rein mechanische Einteilung zu sein, denn es zeigt sich, daß die Zahl der Windungen auf die Größe des Durchmessers bezogen ist: Armspiralen bis zu $5 \mathrm{~cm}$ lichte Weite haben 2-4 Windungen, während Exemplare mit 9 und mehr Windungen einen Durchmesser von mindestens $6 \mathrm{~cm}$ haben $^{115}$. Aus dieser regelhaften Größenrelation darf

III Aus Grab K VII: Andronikos 1969, 234 Abb. 74.

112 Gräber 10 (1921), 13 (1922), 16 (1922), 17 (1922), 18 (1922): S. Casson, Annu. Brit. School Athens 24, 1919-21, 11 mit Abb.11; 26, 1923-25, $9 \mathrm{ff}$.; gleiche Zeitstellung besitzt ein Exemplar aus Visoj: Kilian 1975a, Taf.61,1.

113 Die Geschlossenheit des Grabes K VII von Vergina ist nicht ganz sicher, doch gibt es in Chauchitsa ausschließlich Gräber mit nur einer Nadel.

114 Andronikos 1969, 241.

115 "Klein" sind die Armspiralen aus den Gräbern $\mathrm{A} I X, \mathrm{~N} I$, AE IX, AZI, AZII, AZIV, Malamas B II, Malamas B VII; "große" Armspiralen stammen aus den Gräbern $\mathrm{E} I, \mathrm{~N} I X$, $\mathrm{N} X, \mathrm{O} I, \mathrm{Y} I, \Phi I I I, \mathrm{~A} \Delta I, \mathrm{AE} V, \mathrm{AE} V I I, \mathrm{AZ} V I I, \mathrm{AH} I I$, Malamas B III, Malamas $\Gamma I$. Zwischen diesen beiden Gruppen stehen Armspiralen mit 7 Windungen, die mit $4,4 \mathrm{~cm}$ und mit $6 \mathrm{~cm}$ Durchmesser begegnen: Gräber $\mathrm{N} I V, \mathrm{~N} V I I$, $\mathrm{AB} I$. wohl geschlossen werden, daß in Vergina altersspezifische Formate vorliegen, d.h. niedrige Armspiralen für Jugendliche und hohe für Erwachsene. Eine entsprechende Unterscheidung ist auch in der Nekropole von Vitsa zu beobachten"16. In den Grabfunden begegnen Armspiralen meist einzeln, seltener paarweise oder zusammen mit einem schlichten Armring. Getragen wurden sie vorwiegend am linken Arm ${ }^{117}$; nur in zwei Gräbern ergibt sich aus ihrer Fundlage eine Tragweise am rechten Arm ${ }^{118}$. Kennzeichnend für die Trachtkomposition von Vergina ist die ausschließliche Zugehörigkeit der Armspiralen zu ${ }_{n}$ reichen “ Inventaren, die zumindest aus Fibeln bzw. Nadeln, Kopfschmuck mit Spiralröllchen, Lockenringen und Perlen bestehen. Als einziges Schmuckstück konnten sie, anders als die schlichten Armringe, anscheinend nicht getragen werden ${ }^{119}$.

Durch das Grab N VII mit einer kleinen Bogenfibel sind Armspiralen bereits für die Stufe II der Nekropole nachgewiesen; häufiger treten sie dann während der Phasen III A-C auf. Mit der Stufe IV zeichnet sich ein Trachtwandel ab, bei dem die Armspiralen durch paarweise getragene, schwere Armringe ersetzt werden. Außerhalb Makedoniens, z. B. im Kosovo-Gebiet, bleiben die hohen Armspiralen dagegen noch bis in die Eisenzeit II geläufig ${ }^{120}$. Die schweren, aus einem kräfrigen Bronzestab von D-förmigem bis ovalem Querschnitt hergestellten Armspiralen sind von Makedonien bis Pelagonien und ins Kosovo-Gebiet verbreitet ${ }^{121}$; südlichster Fundort ist bisher Pherai in Thessalien ${ }^{122}$. Die zwei Arm-

116 Armspirale mit 5 Windungen und 4,2 cm Durchmesser in Grab 103, der Bestattung eines 10jährigen Mädchens; Armspirale mit 18 Windungen und $5-7,5 \mathrm{~cm}$ Durchmesser in Grab 113, der Bestattung einer jungen Frau: Vokotopoulou 1986, 149f.; 151 ff. - Andronikos 1969, 243 erklärt die Größe der Armspiralen allein aus der sozio-ökonomischen Position ihrer Trägerin.

117 Einzelne Armspirale links in den Gräbern $\mathrm{A} I X, \mathrm{E} I$, N VII, $\mathrm{N} I X, \mathrm{O} I, \mathrm{Y} I, \mathrm{AB} I, \mathrm{AE} V I I, \mathrm{AH} I I, \mathrm{LXV} \Xi$, Malamas $\Gamma I .-$ Hohe Armspirale links, niedrige Armspirale rechts in den Gräbern NIV, AZIV. - Armspirale links, Armring rechts in den Gräbern AZII, LXV $\Phi$. - Armspirale rechts und links in den Gräbern $A \Delta I, A Z V I I, \Phi I I I$.

118 Einzelne Armspirale rechts: Grab AEV. - Armspirale rechts, Armring links: Grab AZI. - Waren das Linkshänderinnen?

119 Einzige Ausnahme ist das vermutliche Kindergrab N $I$.

120 Suva Reka, Široko, Gräber I,1 und II,3: N. Slavković-Djurić, Glasnik Muzeja Kosova i Metohije 9, 1964, 537 ff.; Kilian 1975 a, 57 f. Taf. 67-71.

121 Vergina: siehe Anm.115; Poteidaia: M.Vickers, Archaia Makedonia 2, 1977, 29 Abb.4,11-14; Kilian 1975 a, Taf. 32,15; Chauchitsa: ebd. Taf. 39,8. - Axioupolis (Bohemitsa), Grab 2: Rey (Anm. 68) 40 ff. Abb. 8,4. - Hagios Panteleimon (Pateli): ebd. 59, Abb.11. - Giannitsa: A. Vavritsas, Arch. Deltion Chronika 26, 1971, 395 Taf. 396,g. - Popadin Dol: M. Garašanin u. a., Les civilisations préhistoriques de la Macédoine/Ausstellung 'Stip (1971) 81 f. Nr.213. - Visoj-Beranci, Grab 16: Kilian a. a. O. Taf.60,5. - Suva Reka, Siroko, Grab II,3: siehe Anm. 120.

122 Kilian 1975 b, 173 Taf. 65,14-16; 1975 a, Taf. 26,10. 
spiralen aus Vitsa in Epirus wurden von Vokotopoulou als Fremdlinge makedonischer Herkunft bewertet ${ }^{123}$. Spiralig eingerollte Enden wie bei dem Exemplar aus Grab 113 von Vitsa sind an Armspiralen von makedonischen Fundorten allerdings nicht üblich. Von diesen nordgriechisch-südbalkanischen Armspiralen heben sich in Mittel- bis Südgriechenland verbreitete Typen deutlich ab: Seien sie aus schmalen Bändern von D-förmigem Querschnitt gewickelt, aus kräftigen Bändern mit plastischer Mittelrippe oder aus flachen Blechbändern, șie haben alle massive, profilierte Enden ${ }^{124}$.

\section{Armringe}

Der Bestand an Armringen aus den Grabungen Andronikos und Petsas ist erstaunlich einheitlich. Soweit Beschreibungen und Photographien ein Bild vermitteln, sind insgesamt nur zwei Typen vertreten: Selten begegnen schwere, rundstabige Ringe mit leicht verjüngten, überstehenden Enden ${ }^{125}$. Die überwiegende Mehrheit sind schmale Reifen von D-förmigem bis spitzovalem Querschnitt mit geraden oder abgerundeten Enden. Je nach der gewünschten Weite des Ringes können die Enden sich gerade berühren, geringfügig überstehen oder den Ringkörper bis zu einer halben Windung übergreifen ${ }^{126}$. Das recht häufig zu beobachtende Engerbiegen solcher Armringe bedeutet zweifellos, daß bei der Herstellung zwischen Ringen für Erwachsene und für Jugendliche nicht unterschieden wurde. Wenn also in den Gräbern Jugendliche mit einem zusammengebogenen Erwachsenenring ausgestattet sind, dann war es entweder Sitte, denselben Armring von Jugend an zu tragen, oder Kindern für die Grablege den Armring eines Erwachsenen zu geben. Zwei weitere, bisher aus Vergina noch nicht bekannte Armringtypen liegen jetzt aus den Gräbern $I I I$ und VI im Hügel B (Feld Malamas) vor. Die Inv. Nr. 843 (Abb. 17,10) ist ein schwerer, rundstabiger Ring mit kleinen, kaum abgesetzten Stempelenden. Bei dem ebenfalls schweren, sechskantigen Ring Inv. Nr. 845 (Abb. 17,16) wird der Querschnitt kurz vor den kleinen Stempelenden achtkantig. Dieser Ring gehört in Vergina zu einem Inventar der Stufe IV. Ein Paar entsprechender Armringe fand sich in dem gleichzeitigen Grab A von. Chauchitsa ${ }^{127}$. Ebenfalls in die Spätphase der Nekropole dürfte schließlich ein offener Armring von rhombischem Querschnitt mit Strichdekor zu datieren sein, der aus einem zerstörten Grab stammt ${ }^{128}$.

123 Vokotopoulou 1986, $317 \mathrm{f}$.

124 z. B. J. Böhlau, Jahrb. DAI 3, 1888, 363 Abb.i; Heurtley u. Skeat 1930-31, 33f. Abb. 14.1-4; I. Konstantinou, Arch. Deltion 18, 1963 Chronika 130 Taf. 164-165; J. Bouzek, Forsch. u. Ber. Staatliche Mus. Berlin 16, 1975, $161 \mathrm{f}$. (Gruppen A-B); H. Philipp, Bronzeschmuck aus Olympia (1981) $205 \mathrm{f}$. (mit weiteren Nachweisen).

125 Aus den Gräbern $A \Delta I, \mathrm{LXV} \Pi$ und Malamas B VI.

126 Ringe aus den Gräbern $\mathrm{N} V, \mathrm{~N} V I, \mathrm{~N} X, \mathrm{~N} X I I, \mathrm{~N} X V I I$, $\mathrm{AB} I I, \mathrm{AE} I, \mathrm{AZII}, \mathrm{AH} V I$; vermutlich auch III $B$, III $\Delta$, $\mathrm{LXV} \Gamma, \mathrm{LXV} A B, \mathrm{LXV} A \Theta$.

127 Kilian 1975 a, 76 Taf. 35,19-20.
In den älteren Gräbern von Vergina (Stufe II) gibt es keine Armringe. Die leichten Ringe mit spitzovalem Querschnitt setzen mit der Phase III A ein und bleiben bis in die Stufe IV geläufig; die schweren Stücke mit Stempelenden dagegen treten erst ab der Phase III C auf. Während der Belegungsdauer der Nekropole von Vergina zeichnet sich ein Wandel in der Tragweise von Armringen ab (Abb. 36-38): In der Stufe III werden sie einzeln getragen, bei reichen Trachtausstattungen manchmal in Kombination mit einer Armspirale, in bescheidenen Inventaren ohne weitere Trachtteile als einziger Schmuck ${ }^{129}$. Ab der Phase III C und insbesondere in der Stufe IV erscheinen Ringe dann sowohl in reichen wie in armen Inventaren in der Regel paarweise $^{130}$.

\section{Faleren}

Grab $\Gamma I$ (Feld Malamas) ist das bisher einzige Grab in der Nekropole von Vergina, das sechs Faleren enthält. Ebenso wie bei den fünf Exemplaren aus Grab AZ VII handelt es sich auch hier um zwar zusammengehörige aber in Form und Dekor nicht völlig übereinstimmende Faleren ${ }^{131}$ : Den Inv. Nr. 858; 859; 861 gemeinsam sind die flache Krempe mit vier getriebenen Buckelkreisen und der konische Knauf, sie unterscheiden sich durch die Form des Mittelbuckels (Abb.26,6.8-9); die kleine Falere Inv. Nr. 860 (Abb. 26,1) hat eine schräge Krempe, gewölbten Mittelbuckel und eine eingesetzte, angegossene Öse ${ }^{132}$; bei der Inv. Nr. 863 mit flacher Krempe dagegen ist die Öse mitgegossen (Abb. 26,2), und die nur flach gewölbte Zierscheibe Inv. Nr. 862 schließlich hat keinen Knauf (Abb. 26,4). Für die viel diskutierte Frage nach der Funktion von Faleren ${ }^{133}$ haben die ungestörten Gräber von Vergina erstmals gesicherte Fundbeobachtungen erbracht. Sie begegnen dort nie zusammen mit Waffen, sondern stets in Kombination mit einer ziemlich reichen Schmuckausstattung. Sie liegen einzeln, paar-

128 Rhomiopoulou (Anm. 80) 13 f. Abb. 2-3; zu Datierung und Verbreitung siehe Kilian 1975 a, 109 Taf. 86.

129 z. B. Gräber N $I X, \mathrm{~A} \triangle I I, \mathrm{AZI}, \mathrm{AZ} I I, \mathrm{LXV} \Phi$ bzw. N $X I I$, NXVII, AH VI, LXVAZ.

130 z. B. Gräber III $B$, III $\Delta$, LXV T, Malamas B VI bzw. AH IX.

131 Im Grab AZ VII sind es zwei gleichgroße Bronzefaleren mit unterschiedlichen Knäufen $\left(\pi, \pi^{2}\right)$, eine größere Bronzefalere mit hohem, konischem Buckel $(\rho)$, eine kleine Bronzefalere mit gewölbtem Buckel $\left(\omega^{\prime}\right)$ und eine kleine Eisenfalere $(\sigma)$ : Andronikos 1969, 144ff.; 244f.; Abb. 84-85; Taf. 127-129.

132 Zum technischen Verfahren siehe B. Fellmann, Frühe olympische Gürtelschmuckscheiben aus Bronze (1984) $46 \mathrm{ff}$. Im Gegensatz zu den bei Fellmann behandelten Faleren sind bei den Exemplaren von Vergina Knauf, Buckel und Krempe aus einem Stück gearbeitet und nur die Öse ist eingesetzt.

133 z. B. G.v. Merhart, Jahrb. RGZM 3, 1956, 28 ff.; Andronikos 1969, 159; 243 ff.; A.Snodgrass, Hamburger Beitr. Arch. 3, 1973, 41 ff.; P. Zancani Montuoro, Atti Memorie Società Magna Grecia N.S. 15-17, 1974-76, 83 ff.; Fellmann (Anm. 132) mit älterer Lit.; zuletzt Vokotopoulou 1986, $304 f$. 


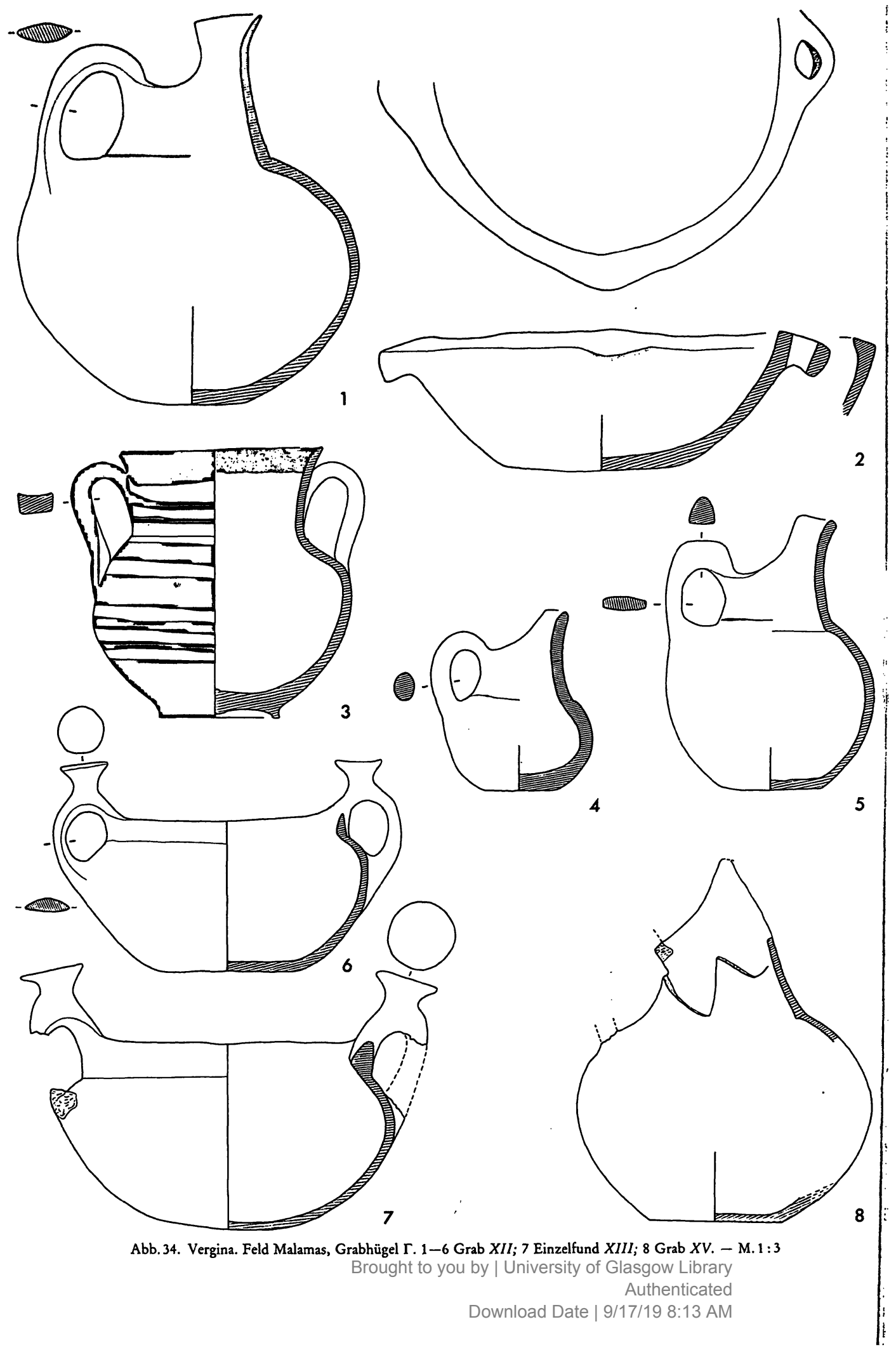




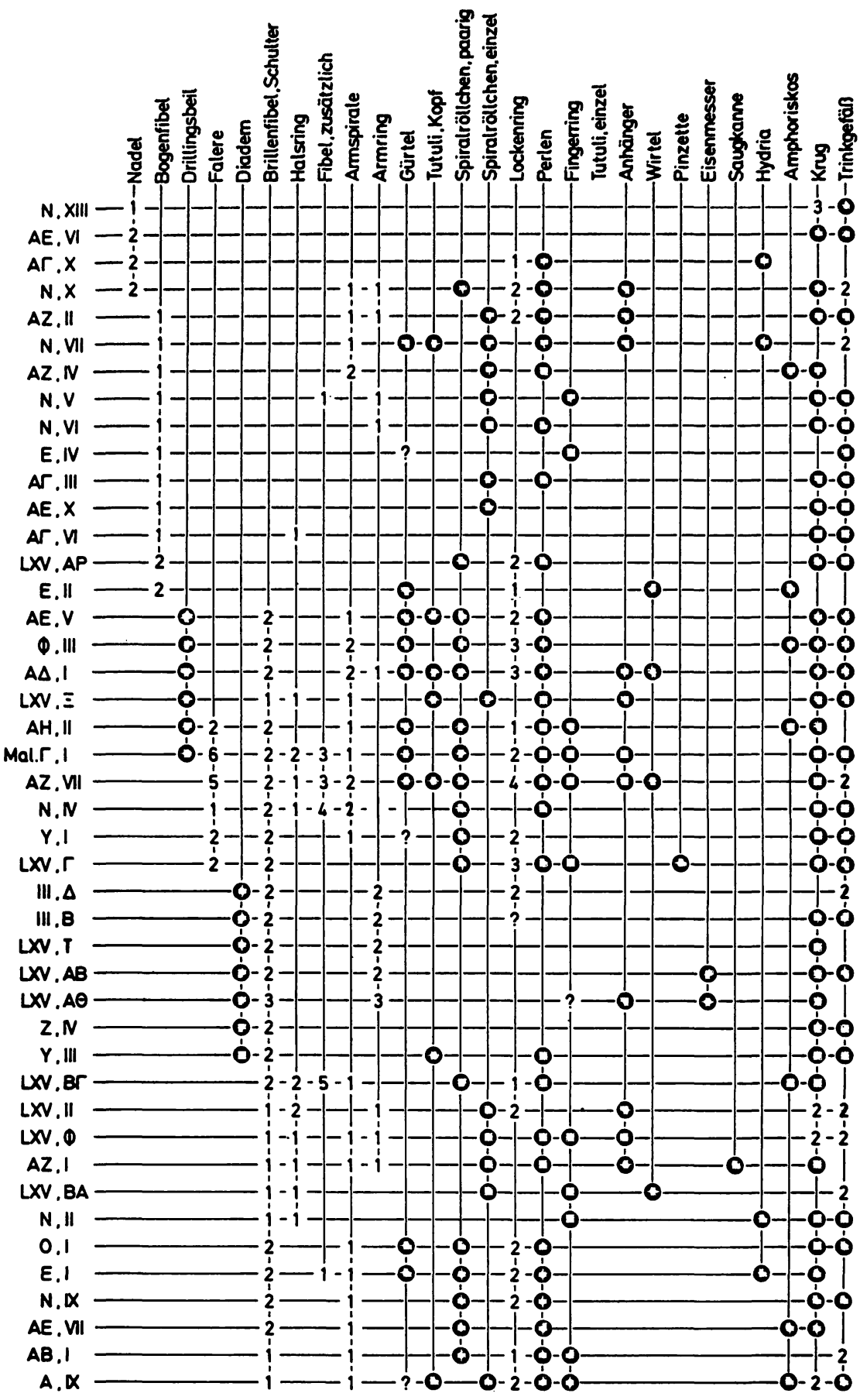

Abb.36. Vergina. Beigabenkombination in vermutlichen Frauengräbern (siehe auch Abb.37-38) 


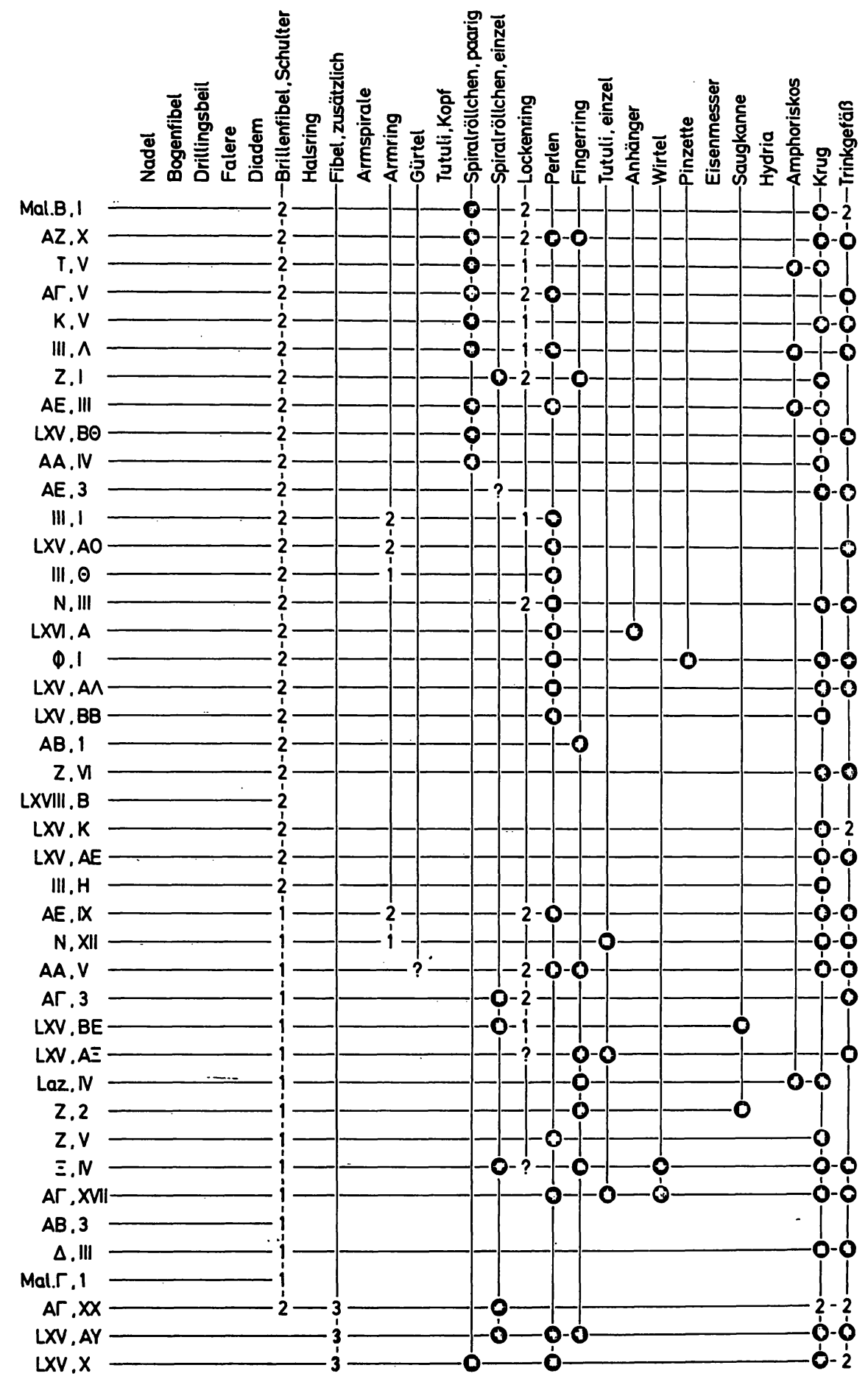

Abb. 37. Vergina. Beigabenkombination in vermutlichen Frauengräbern (siehe auch Abb. 36; 38) 


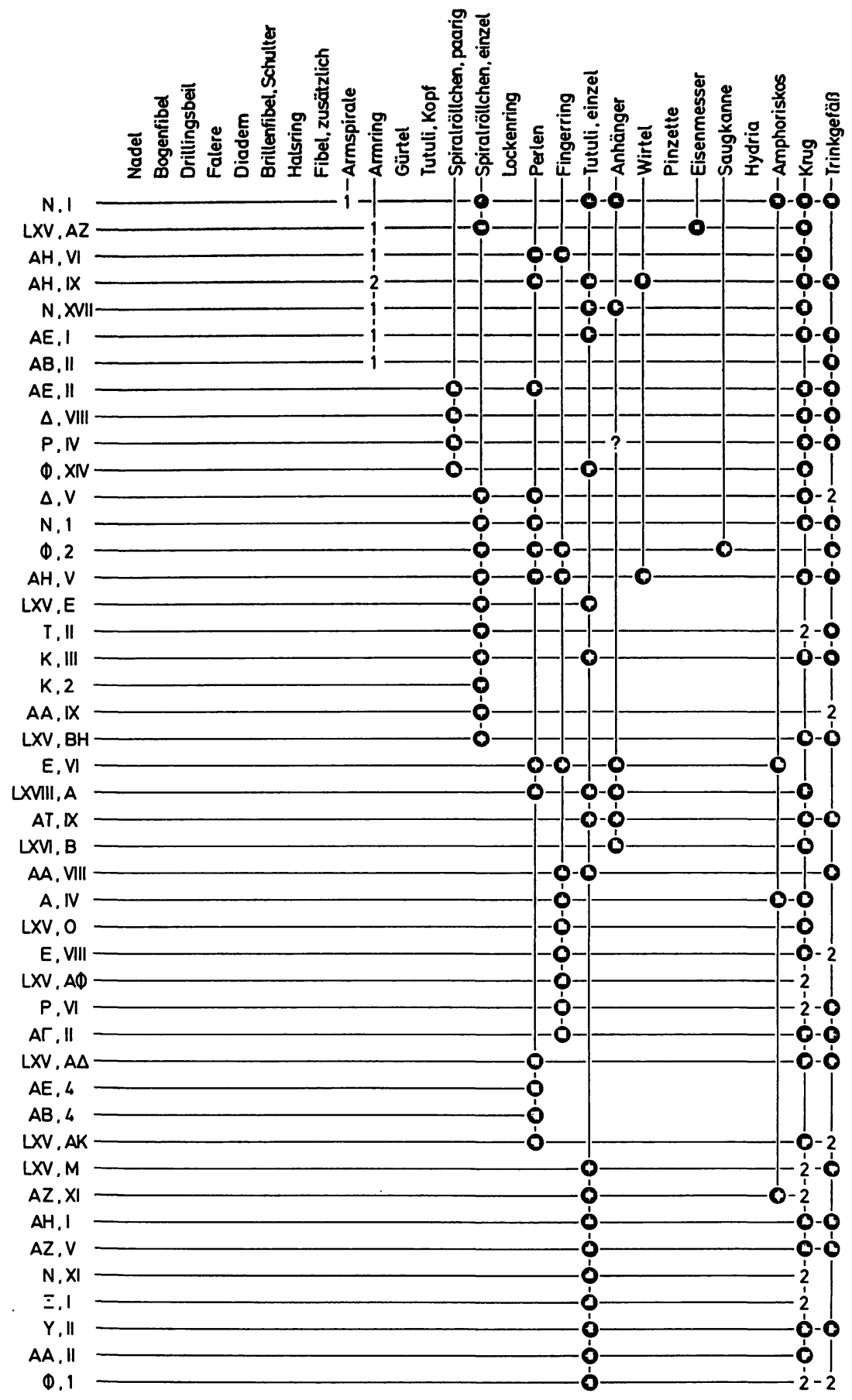

Abb. 38. Vergina. Beigabenkombination in vermutlichen Frauengräbern (siehe auch Abb. 36-37) 


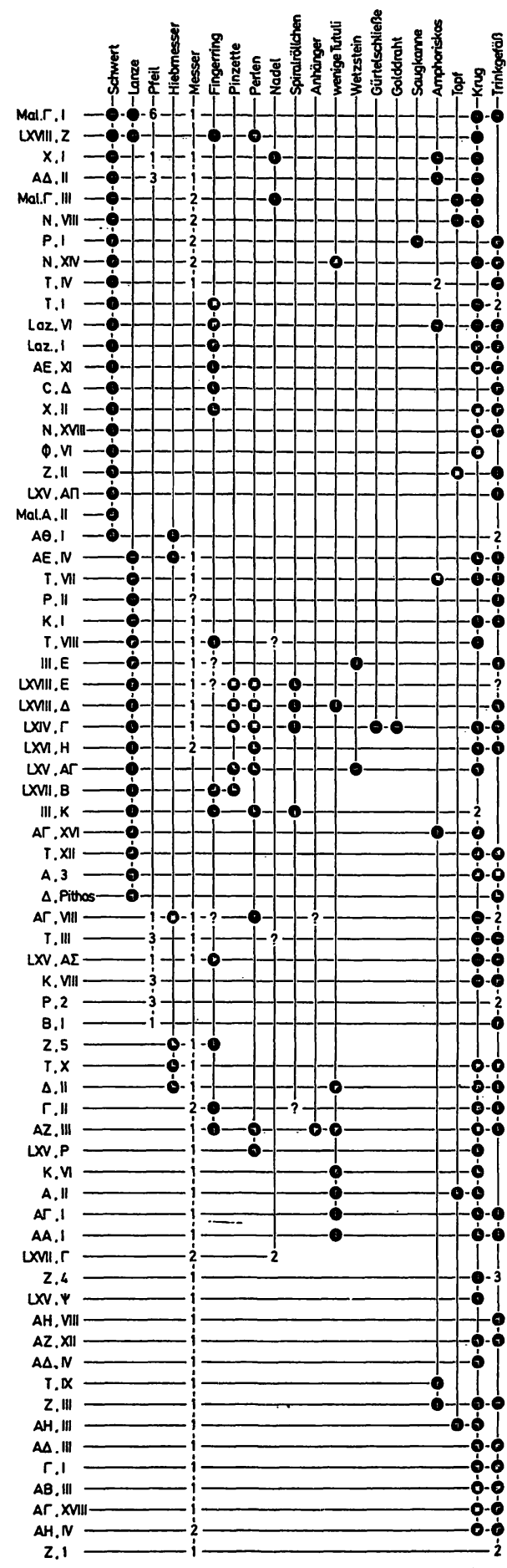

Abb. 39. Vergina. Beigabenkombination in Gräbern mit Waffenbeigabe und in vermutlichen Männergräbern weise oder zu dritt in der Grabmitte, manchmal in unmittelbarer Nähe von zahlreichen kleinen Tutuli ${ }^{134}$. Aus diesem Befund erschloß Andronikos eine in Vergina getragene Frauentracht, deren tutulusbesetzter Gürtel vorne in der Leibesmitte mit ein bis drei Faleren geschmückt war ${ }^{135}$. Diese für Vergina aufgezeigte Verwendung halten Andronikos und nach ihm Fellmann auch bei der Mehrzahl der Faleren aus griechischen wie außergriechischen Fundorten für zutreffend ${ }^{136}$. Anzahl und Verteilung der Faleren im Grab $\Gamma I$ (Feld Malamas) stellen die Gültigkeit einer derartigen Interpretation jedoch in Frage. Die in sechs parallelen Reihen geführten Tutuli liegen dort mit der Öse nach unten, sie waren also auf der Vorderseite des Gürtels - nicht nur auf dem Rückenteil ${ }^{137}$ - befestigt. Aus ihrer intakten Anordnung geht eindeutig hervor, daß die Position der drei Faleren (Inv. Nr. 858; 859; 860) nahe bei den Tutuli nicht das Ergebnis einer sekundären Verlagerung ist. Bei der Tracht der Toten in Grab $\Gamma$ I (Feld Malamas) dienten die Faleren folglich nicht als Gürtelschmuck, sondern als Kleiderbesatz, wobei eine Falere oberhalb des ringsum mit Tutuli besetzten Gürtels auf dem Oberkörper (im Rippenbogen) sitzt, zwei unterhalb des Gürtels etwa in Höhe des Beckens ${ }^{138}$ und zwei weitere ungefähr in Kniehöhe. In Vergina war eine derartige Tragweise der Faleren offensichtlich die Regel und keine seltene Ausnahme, wie eine Überprüfung aller Gräber mit Faleren deutlich macht. Im Grab AZ VII liegen drei große Faleren in deutlichem Abstand unterhalb des Gürtels ${ }^{139}$. Zusätzlich zu diesen fanden sich noch eine vierte kleinere aus Eisen im Bereich des linken Oberschenkels sowie zwei Besatzbuckel zusammen mit einem Radanhänger in der Gegend des rechten Knies. Auch im Grab AH II ist die Position des Gürtels durch die Tutuli seines Besatzes markiert ${ }^{1+0}$. In ihrer ursprünglichen Lage unterhalb des Gürtels dürfte sich die Falere auf der linken Körperseite befinden, während ihr Gegenstück der rechten Seite zusammen mit einigen Tutuli vom Gürtelbesatz sekundär verlagert sein könnte. Der einzelne Tutulus in Grab Y I, der wohl die einzige Bronzezier eines Gürtels aus vergänglichem Material bildete, und der Abstand zwischen Armspirale und Faleren sprechen dafür, daß hier die gleiche paarweise Anordnung unterhalb des Gürtels vorliegt wie

134 Eine Falere: Grab NIV; zwei Faleren: Grab YI, AHII, LXV $\Gamma$; drei (plus zwei) Faleren: Grab AZ VII; drei (plus drei) Faleren: Grab Malamas $\Gamma I$.

135 Andronikos 1969, 159; $243 \mathrm{ff}$.

136 Ebd. 247; Fellmann (Anm. 132) $110 \mathrm{f}$.

137 Andronikos 1969, 159 Anm. 1 beschreibt den Gürtel im Grab AZ VII mit Tutulusbesatz nur seitlich und hinten, während vorne die Faleren sitzen.

138 Es sei besonders darauf hingewiesen, daß die eine der Faleren von der untersten Tutulireihe überlagert wird, d.h. der untere Rand des Gürtels hat sich leicht über die Krempe der Falere geschoben.

139 Ebd. 75; Taf. K; 27-29.

10 Ebd. 78; Abb. 20; Taf. 29. 
im Grab $\Gamma I$ (Feld Malamas) ${ }^{14}$. In den Gräbern N $I V$ und LXV $\Gamma$ bleibe das Verhältnis zwischen Faleren und hypothetischen Gürteln unklar ${ }^{142}$. Für Vergina ist damit dic Funktion der Faleren als Klciderbesatz gesichert. Häufig werden sic paarweise auf dem Unterleib getragen, sic können aber auch an anderen Körperstellen angebracht sein, wie z. B. auf der Hüftc, auf dem Oberkörper, in Höhe der Knic. Die glciche Verwendung von Kalottenbuckeln und Krempenfalcren, d.h. an Schulter, Brust und Hüfte auf das Gewand aufgenäht, ist in der Basilikata durch Frauengräber des 9.-8. Jhs. v. Chr. belegt ${ }^{143}$. In Epirus dagegen enthielten die ins 8. Jh. v. Chr. datierten Männergräber 34 und 79 von Vitsa jeweils eine Krempenfalere mit profiliertem Knauf ${ }^{1+4}$. Aus ihrer Fundlage auf dem Schädel des Bestatteten wird deutlich, daß sie dort Bestandteil einer Kopfbedeckung waren ${ }^{145}$. Die bronzenen Helmteile aus dem protogeometrischen Grab XXVIII von Tiryns müssen, wie bereits N. Verdelis in einer Rekonstruktionszeichnung veranschaulicht hat, so auf einer Stoff- oder Lederkappe befestigt gewesen sein, daß zwischen ihnen ein von der Stirn bis in den Nacken reichender Scheitelstreifen frei blieb ${ }^{146}$. Da die eine Falere dieses Grabes unmittelbar neben den Seitenblechen des Helms lag, könnte auch sie als Scheitelbesatz zum Helm gehört haben. Es liegt nahe, dann auch in den Faleren aus den protogeometrischen Gräbern 24, 40 und 43 im Kerameikos den Besatz einer helmartigen Kopfbedeckung zu vermuten, mit der die Mündung der Grabamphore verschlossen war ${ }^{147}$. Mit diesen aus der Interpretation von Grabfunden gewonnenen Hinweisen auf die Funktion von Faleren zeigt sich erneut, daß mit regional wie zeitlich differenzierten Verwendungsarten gerechnet werden muß ${ }^{1+8}$.

Morphologisch gliedern sich die Faleren aus der Nekropole von Vergina in drei Typen: 1. mit kalottenförmigem Buckel; 2. mit flachem Buckel; 3. mit hohem konischen Buckel auf Trommel ${ }^{149}$. Diese Formunterschiede haben offensichtlich keinen chronologischen Aussagewert, da der Typ mit Kalottenbuckel ebenso in dem

${ }^{1+1}$ Ebd. 50 f.; Abb. 14; Taf. 16 (Tutulus $\lambda$ zwischen Armspirale und Faleren).

$1+2$ Grab NIV mit einer Falere: Ebd. 33; Abb. 5; Taf. 12 . Grab LXV $\Gamma$ mit zwei Faleren: Petsas 1961-62, 269f.; Taf. $132, \gamma ; 133, \gamma$.

${ }^{143}$ z. B. Metapont, Incoronata Gräber 23, 63, 135: B. Chiartano, Metaponto II. Not. Scavi Suppl.31, 1977, 134 ff. Abb.76; $166 \mathrm{ff}$. Abb. 96; $176 \mathrm{ff}$. Abb. 105. - Tursi, Valle Sorigliano, Grab 28: S. Bianco in: Siris-Polieion/Incontro Studi, Policoro 1984 8-10 giugno (1986) 24 Taf. 8.

14 Vokotopoulou 1986, 83 ff.; 102 f.; Taf. 120-121; 149-150.

145 Ebd. 304 f.; vgl. dagegen Snodgrass (Anm. 133) 47 f., der die Falere aus Grab 34 als Schildbuckel erklärt.

146 Verdelis (Anm. 75) $10 \mathrm{ff}$. Abb. 9.

147 Kübler 1943, $27 \mathrm{ff}$; $41 \mathrm{ff}$.

148 So bereits Snodgrass (Anm. 133) 48; anders dagegen Fellmann (Anm. 132) $109 \mathrm{ff}$., der eine generelle Verwendung als Gürtelbesatz postuliert.

149 Andronikos 1969, 244f. Abb. 84-85. frühen Grab N IV (Phase III A) vorkommt wie in dem jüngeren Grab Malamas $\Gamma I$ (Phase IIIC), und Grab AZ VII (Phase III B) Falcren aller drei Formen enthält. Durch die schlichten, niedrigen Knäufe und vor allem in der Herstellungstechnik - Knauf und Scheibe sind in cincm Stück gearbeitet und nur die Ose ist meist eingesetzt ${ }^{150}$ - unterscheiden sich die Faleren aus Vergina von den gleichzeitigen Exemplaren des südlichen Griechenland'151. Für ihre Herstellung in einer Werkstatt der Region spricht auch der gleichartige Buckeldekor auf den Krempen ${ }^{152}$. Mit der Falere Inv. Nr. 861 (Abb. 26,6) liegt eines der wenigen Beispiele für eine antike Reparatur vor 153: Ohne jeden technischen Aufwand ist hier eine Bruchstelle am Mittelbuckel mit Hilfe von zwei Drahtklammern geflickt. Die primitive Ausführung macht es unwahrscheinlich, daß es sich um einen Herstellungsfehler handelt, der noch in der Werkstatt von einem Bronzeschmied behoben wurde. Wenn demnach die Beschädigung durch den Gebrauch entstanden sein muß, bedeutet sie zugleich, daß die Faleren nicht für die Grablege gefertigt wurden, sondern als Bestandteil einer tatsächlich getragenen Tracht.

Drillingsbeile

Aus Grab Malamas $\Gamma I$ liegt nun das sechste Drillingsbeil von Vergina vor (Abb. 26,7) ${ }^{15+}$. Mit den geschlossenen, geraden Längsseiten, den ausgeschnittenen Dreiekken $z$ wischen den Beilklingen und den Kreisscheiben als Bekrönung bildet es eine nahe Entsprechung zu dem Exemplar aus Grab AE $V$, allerdings ohne geritzten Dekor. Erstmalig sind an diesem Stück Reste eines Schaftes oder Griffs erhalten, ein kräftiger Eisenstab, der mit dem Stiel der Doppelbeile vernietet ist. Damit bestätigt sich auch Andronikos' Ansicht, daß diese Doppelbeile keine Anhänger waren, sondern in der Hand getragene Gegenstände $^{155}$. Ebenso wie in den Gräbern $\Phi I I I$ und LXV $\Xi$ lag das Drillingsbeil auch im Grab Malamas $\Gamma I$ auf der rechten Seite des Oberkörpers, die rechte Brillenfibel teilweise verdeckend, mit dem Griff in Richtung zum Kopf (Abb. 20). In den Gräbern $A \triangle I, A E V$ und $\mathrm{AH} I I$ dagegen fand es sich rechts bzw. links neben dem Kopf und mit dem Griff zur Schulter. Diese unterschiedliche Orientierung könnte bedeuten, daß für die Grab-

150 Ausnahme die kleine Falere Inv. Nr. 863 aus Malamas $\Gamma I$.

151 Überblick über die Funde in Griechenland: Fellmann (Anm. 132) $3 \mathrm{ff} . ; 68 \mathrm{ff}$.

152 Einer anderen Werkstatt wäre wohl die Falere in Grab 2 von Axioupolis (Bohemitsa) zuzuweisen, die unverziert ist und keinen Knauf sondern ein Scheitelloch hat: Rey (Anm. 68) $40 \mathrm{ff}$. Abb. 8,8.

${ }_{153} \mathrm{Zu}$ Reparaturen von Faleren siehe Fellmann (Anm. 132) $51 \mathrm{f}$.

15t Fünf Exemplare aus den Gräbern $\Phi I I I, \mathrm{~A} \Delta I, \mathrm{AE} V, \mathrm{AH} I I$, LXV $\Xi$ : Andronikos 1969, $248 \mathrm{ff}$. Abb. 87; Petsas $1961 \div 62$, 274 f.; Taf. 134; 150,a; Kilian-Dirlmeier 1979, $244 \mathrm{f}$. Nr. 1564-1568.

155 Andronikos 1969, 160; 250; unzutreffend den Anhängern zugewiesen von Kilian-Dirlmeier 1979, $244 \mathrm{f}$. 
lege die Ausrichtung des Drillingsbeils nicht notwendig seiner realen Handhabung entsprechen mußte. Der gleiche Befund wäre aber auch zu erwarten, wenn die Drillingsbeile ursprünglich senkrecht an der Schulter der Toten lehnten und erst mit dem Vergehen der Leiche nach vorne (auf die Brust) oder nach hinten (neben den Kopf) kippten. Die Drillingsbeile sind in Vergina eine Beigabe, die nur in Gräbern mit besonders reicher Schmuckausstattung vorkommt ${ }^{156}$. Aus ihrer regelhaften Kombination mit zwei Brillenfibeln, ein bis zwei Armspiralen, tutulusbesetztem Gürtel, Kopfbinde aus Spiralröllchen und goldenen Lockenringen darf wohl eine statusrelevante Bedeutung erschlossen werden (Abb. 36).

In einem Stück gefertigte Drillingsbeile sind bisher nur aus Vergina bekannt. In Nordmakedonien enthalten ein Grab von Kumanovo und Grab 16 von Beranci je drei bronzene Doppeläxte mit Schaftloch ${ }^{157}$. Auf einen Holzstab aufgeschoben könnten auch sie Drillingsbeile gebildet haben. Die drei Doppelbeile aus Grab A von Spilaion in Westmakedonien haben Ösen und wurden vielleicht als Anhänger getragen ${ }^{158}$. Ein einzelnes Doppelbeil gleicher Form mit Öse lag in Grab 21 von Vitsa, Epirus, zusammen mit zwei eisernen Brillenfibeln und einem kleinen Eisenmesser auf der rechten Schulter ${ }^{159}$. Mit diesen Funden zeichnet sich eine weitere Verbreitung von Drillingsbeilen und Doppelbeil-Anhängern $a b$, die bis Nordmakedonien, Pelagonien und Epirus reicht. Läßt sich das Grab. A von Spilaion im Reichtum seiner Schmuckbeigaben mit Vergina vergleichen, so sind die Inventare von Kumanovo, Beranci und auch Vitsa merklich bescheidener; es sind jedoch in allen Regionen Frauengräber, in denen Doppelbeile begegnen ${ }^{160}$.

\section{Anhänger}

Anhänger gehören in Vergina zu den seltenen, erst in den fortgeschrittenen und späten Phasen der Belegungszeit auftretenden Beigaben. Im Grab Malamas $\Gamma I$ wiederholt sich die bereits aus Grab AZ VII bekannte Kombination von Ring- und Radanhänger (Abb. 26,3:5) ${ }^{161}$. Übereinstimmend ist auch die Fundlage: In Malamas $\Gamma I$ überlagert der Ringanhänger etwa in Höhe der rechten Schulter den Halsring, während der Radanhänger unterhalb des Gürtels im Bereich der Knie liegt (Abb. 20). Im Grab AZ VII fand sich der Ringanhänger unmittelbar neben der unteren Spirale der rechten Brillenfibel und

is6 Andronikos 1969, 160.

157 Kilian-Dirlmeier 1979, 244 Nr. 1569-1570.

158 Rhomiopoulou (Anm.67) 37ff. Abb.4; Kilian-Dirlmeier 1979, 246 Nr. 1577-1579.

159 Vokotopoulou 1986, 69ff.

160 In Vitsa anthropologische Bestimmung: Ebd.69; für die übrigen Gräber aufgrund der Beigaben. Zur zweifelhaften Zugehörigkeit einer Lanzenspitze zu Beranci, Grab 16 vgl. Kilian 1975 a, 82.

161 Andronikos 1969, 75 f.; Taf. 125, $\varepsilon ; 129, \omega^{n}$. der Radanhänger in der Nähe des rechten Knies ${ }^{162}$. Um zwei Anhänger zu tragen, wurde also der cine an den Hals, der andere an den Gürtel gehängt oder vielleicht auf das Gewand aufgenäht ${ }^{163}$. Beide Tragweisen sind auch für einzelne Anhänger möglich, denn im Grab $N X$ lag ein Radanhänger beim Hals und im Grab LXV $\Phi$ ein Ringanhänger auf dem Unterkörper ${ }^{164}$.

Die beiden Anhänger von Malamas Grab $\Gamma I$ gehören weit verbreiteten Typen an, die über Makedonien hinaus auf dem Balkan bzw. im südlichen Griechenland vorkommen ${ }^{165}$.

\section{Kleine Bronzeringe}

Unter den zahlreichen kleinen Bronzeringchen lassen sich nach Herstellungsart und Form vier Gruppen unterscheiden $^{166}$ :

1. Gegossene, geschlossene Ringe von dreieckigem oder plankonvexem Querschnitt (z.B. Abb.6,2-3; 17,20-22),

2. offene, aus einem Band von D-förmigem Querschnitt zusammengebogene Ringe (z. B. Abb. 30,15-16),

3. Ringe mit gegenständigen Spiralenden (Abb.6,5.7; 25,16-17),

4. aus Fragmenten in sekundärer Verwendung hergestellte Ringe (Abb. 30,10).

Nach ihrer Fundlage in den Gräbern wurden solche Ringe in Vergina entweder am Finger oder in Kombination mit Spiralröllchen auf dem Kopf getragen. Fingerringe, teils einfache Reifen, teils Ringe mit Spiralenden, sind der einzige Schmuck, der in Waffengräbern begegnet (Abb. 39) ${ }^{167}$. Die gleichen Ringformen finden sich aber auch in Frauengräbern und zwar sowohl an der Hand ${ }^{168}$ als auch am Kopfschmuck ${ }^{169}$. Gegossene Ringe mit plankonvexem Querschnitt sind auch in Griechenland südlich von Makedonien verbreitet; man datiert sie

162 Ebd. 76 Taf. K. Da im Grab AZ VII der Kopfschmuck aus Spiralröllchen anscheinend sekundär in Richtung auf den Unterkörper verlagert ist, könnte auch der Ringanhänger ursprünglich näher beim Halsring gelegen haben.

${ }^{163}$ Ein Gürtelgehänge mit bronzenen Anhängern dürfte z. B. in Axioupolis (Bohemitsa), Grab 2; Chauchitsa, Grab 8; Kuç i Zi, Grab 14; Vitsa, Grab 113 vorliegen: Rey (Anm. 68) 40 ff.; S. Casson, Annu. Brit. School Athens 26, 1923-25, $7 \mathrm{f}$.; Zh. Andrea, Iliria 6, 1976, 172; Vokotopoulou 1986, $151 \mathrm{ff}$.

164 Andronikos 1969, 35 f.; Petsas 1961-62, 276 Abb. 55.

165 Weitere Nachweise Kilian-Dirlmeier 1979, 9 f.; $23 \mathrm{f}$.

166 Siehe auch Andronikos 1969, 240 f. Abb. 80-81.

167 z. B. Grab T I, T VIII, XII, AE XI, LXVIII Z, C 4 , Lazaridis $I$, Lazaridis $V I$; vermutlich auch Männergräber: Malamas III, AZIII.

168 z.B. Grab N VII, NIX, AH II, LXV $\Xi$, Malamas $\Gamma I$.

169 Ring mit gegenständigen Spiralenden beim Kopf: Grab AB I; vermutlich ebenfalls zum Kopfschmuck gehörig im Grab AZ VII. - Bis zu vier Ringchen beim Kopfschmuck: z. B. Grab $\mathrm{E} I, \mathrm{~N} I V, \mathrm{~N} I X, \mathrm{~N} X, \mathrm{Y} I, \Phi I I I, \mathrm{AB} I, \mathrm{AH} I I, \mathrm{AZ}$ $V I I$, Malamas $\Gamma I I$. 
in submykenische bis geometrische Zcit ${ }^{170}$. Außerhalb Makedoniens kann bisher nur cine liunktion als Fingerring nachgewiesen werden ${ }^{\prime \prime \prime}$.

$\mathrm{Zu}$ den Fingerringen mit Spiralenden vgl. I. KilianDirlmeicr, Jahrb. RGZM 27, 1980, 249 ff.

\section{Schwerter}

Neben Athen, Lefkandi, Eretria und Knossos zählt Vergina zu den Fundorten mit cinem reichen Bestand an eisernen Griffzungenschwertern ${ }^{12}$. Eine typologische und chronologische Einteilung der Schwerter von Vergina hat K. Kilian vorgelegt $t^{173}$. In der Ausbildung des Griffs unterschied er Griffzungen mit annähernd parallelen Rändern (wie Grab XII, C $\Delta$ ), gebauchte Griffzungen (wie Grab $\mathrm{AE} X I, \mathrm{X} I, \mathrm{~A} \Delta I I$ ) und am Übergang zur Schulter kantig einziehende Griffe (wie Grab $\mathrm{N} X I V$, $\mathrm{N} X V I I I, \mathrm{LXV} A I)$. Da die Griffbildung mit geraden oder nur wenig ausbiegenden Rändern in der Tradition spätmykenischer Bronzeschwerter des Typus Naue II steht ${ }^{174}$, dürfte diese Ausführung in Eisen auch chronologisch die ältere sein und somit in die Frühstufe der Nekropole zu datieren; die beiden anderen Formen gehören dann den folgenden Phasen III A und III B an. An den Eisenschwertern aus Mittelgriechenland, die durch ihre Beifunde in das feinteilige Chronologieschema der Keramik eingeordnet sind, läßt sich eine vergleichbare Formentwicklung nicht aufzeigen ${ }^{175}$. Schwertgräber submykenischer Zeit liegen von dort nicht vor; als Beigabe im Grab setzen Eisenschwerter im Athener Kerameikos erst mit den frühprotogeometrischen Zeitstufen 3-4 (nach Krause) ein. Es sind relativ kurze Schwerter (Länge ca. $50 \mathrm{~cm}$ ) mit unterschiedlich geformten Griffzungen ${ }^{176}$. Spätprotogeometrische Schwerter aus Athen und Lefkandi haben leicht gebauchte Griffzungen mit geradem Abschluß oder Fischschwanzende ${ }^{17}$. In Gräbern frühgeometrischer Zeit

170 z. B. Lefkandi I, 247 f.; Kraiker u. Kübler 1939, 85 f.; C. G. Styrenius, Submycenaean Studies (1967) 48; 70; 109; Verdelis (Anm.75) 7 ff.; Courbin (Anm.75) 133; Vokotopoulou 1986, $313 \mathrm{f}$.

171 Siehe Anm. 170.

172 Müller-Karpe 1962, $59 \mathrm{ff} . ;$ Lefkandi I, $253 \mathrm{f}$. - Eretria III: C. Bérard, L'Herôon à la porte de l'ouest (1970) $13 \mathrm{ff}$. Knossos, Nord-Nekropole: unpubliziert; für die Erlaubnis, die Funde im Museum Heraklion zu sehen, danke ich $\mathrm{H}$. W. Catling, für vielfältige Auskünfte über die Gräber wird A. Snodgrass gedankt.

173 Kilian 1975 a, $67 \mathrm{ff}$.

174 z. B. Karo (Anm. 76) 135 Beil. 37; Th. Papadopoulos, Annu. Brit. School Athens 79, 1984, 221 ff. Abb. 2; K. Demacopoulou, Athens Ann. Arch. 2, 1969, 226 ff. Abb. 1-2; V. Milojčić, Jahrb. RGZM 2, 1955, 159 ff. Abb. 3,4.8; L. Morricone, Annu. Scuola arch. Atene 43-44, 1965-66, $136 \mathrm{ff}$. Abb. 122-124; Ch. Kardara, Aplomata Naxou (1977) 8 Taf. 7,d-e.

175 Dazu zuletzt H.W. Catling in: Lefkandi I, $253 \mathrm{f}$.

176 Gräber PG 6, 2 (Nord) und wohl auch E: Müller-Karpe 1962, 91 Abb. 9.

177 Ebd. 92 Abb. 10,1; Lefkandi I, Taf. 174,14.4; 183,26.18. begegnen mehrfach Schwerter, deren gebauchte Griffzunge nach oben in flachem Bogen oder stumpfwinkelig abschließt ${ }^{178}$. Ans Ende der geometrischen Zeit gehören Schwerter aus Eretria und Hagios Georgios (Thessalien), deren Merkmale eine recht breite, lange Klinge und Randleisten sind, die am Griffende lange, rechtwinkelig abstehende Ohren bilden ${ }^{17}$. Der schlechte Erhaltungszustand der meisten Eisenschwerter macht die genaue Bestimmung von Formdetails und damit dic Definition von Typen unmöglich. Mit den heute zur Verfügung stehenden Funden zeichnen sich jedoch merkliche regionale Unterschiede in der Produktion der Typ II-Schwerter (nach Naue) ab, so daß Einzelvergleiche etwa zwischen Attika und Makedonien kaum für chronologische Einordnungen ausgewertet werden können. Die Schwerter aus den Hügeln Lazaridis, Gräber $I$ und $V I$, und Malamas A, Grab II (Abb. 6,1.4; 7,15) sind wohl an jene aus den Gräbern $\mathrm{X} I, \mathrm{~A} \Delta I I$ und $\mathrm{AE} X I$ anzuschließen ${ }^{180}$ und in die Phase III A der Nekropole zu datieren. Der sehr gedrungene Griff mit geradem Abschluß des Schwertes aus Lazaridis, Grab VI, findet eine Entsprechung bei dem Schwert aus dem spätprotogeometrischen Grab 26 der Toumba-Nekropole von Lefkandi' ${ }^{181}$. Das Schwert aus Malamas Grab ГIII (Abb.30,18) wird durch seine Grifform der Phase III B zugewiesen ${ }^{182}$. Das durch seine Beifunde in die Phase III C datierte Schwert aus Malamas $\Gamma$ I (Abb. 25,1) ist in der Schulter-Griffpartie so schlecht erhalten, daß ein Formvergleich nicht möglich ist.

Die Sitte der Schwertbeigabe wird in Vergina während der gesamten Belegungsdauer der Nekropole geübt. Kennzeichnend für die Schwertgräber aller Phasen ist die bescheidene und kaum variierende Ausstattung (Abb. 39). Als einziges Schmuckstück begegnen Fingerringe ${ }^{183}$; zur Kleidung gehörige Metallgegenstände und als Repräsentationsobjekte klassifizierbare wertvolle Beigaben fehlen gänzlich. Die mehrfach mit dem Schwert kombinierten kleinen Eisenmesser sind wohl Gebrauchsgegenstände des täglichen Lebens. Bei den Pfeilen, die bis zu sechs Exemplaren beigegeben werden konnten, dürfte es sich eher um Jagdwaffen handeln, da ihre zierlichen

178 Kerameikos, Grab G 38: Müller-Karpe 1962, 105 Abb. 23,1. - Agora, Grab 3: Müller-Karpe 1962, 110 Abb. 28,1. - Athen, Odos Kavalotti, Grab $\Delta$ : O. Alexandri, Arch. Deltion 20, 1965 Chronika 75 f. - Lefkandi, Toumba Brandplatz 8: Lefkandi I, Taf. 192,8.4.

179 Eretria III: C. Bérard (Anm. 172) 13 ff.; Abb. 3; Taf. 9,37; A. Tziaphalias, Athens Ann. Arch.11, 1978, $156 \mathrm{ff}$. Abb. 6,17.

180 Andronikos 1969, 262 f. Abb. 101-102.

181 Lefkandi I, Taf. 183,26.18.

182 Vgl. die Schwerter aus den Gräbern NXIV, NXVIII, LXV A I : Andronikos 1969, 262 Abb. 101; Petsas 1961-62, 285 Taf. 152, $\delta$.

183 Singulär unter den Schwertgräbern ist LXVIII Z mit einer Bronzeperle. Nach Ausweis der Gräber mit Lanzenspitze wird die Beigabe von Perlen in Männergräbern erst mit der Stufe IV A üblich. 
und dünnen Spitzen für den Kampf nicht geeignet erscheinen. Auffällig ist die seltene und nur für die Spätphase der Nekropole belegte Kombination von Schwert und Lanze. Falls für die Grabausstattung die reale Bewaffnung bestimmend war, dann müßte das auf Kampftechnik und kriegerische Organisation bezogen eine strikte Trennung von Schwert- und Lanzenkriegern bedeuten ${ }^{184}$. In diesem archäologischen Befund könnte aber auch eine beabsichtigte Beigabenauswahl faßbar werden, bei der die kriegerische Funktion des Toten bereits durch eine einzelne Waffe hinreichend charakterisiert war. Aus der Fundlage im Grab PI kann eine $\mathrm{O}-\mathrm{W}$ orientierte Bestattung erschlossen werden mit dem Saugkännchen beim Kopf, dem Schwert und zwei kleinen Messern an der rechten Seite und dem Skyphos bei den Füßen ${ }^{185}$. Da der Abstand zwischen beiden GefäBen nur $1,50 \mathrm{~m}$ beträgt und Saugkännchen vermutlich eine spezielle Beigabe jugendlicher Personen sind ${ }^{186}$, wäre für das Grab PI die Bestattung eines Jugendlichen anzunehmen. Auch in den Gräbern $\mathrm{XI}, \mathrm{X} I I$ und $\mathrm{N} X I V$ spricht der geringe Abstand $(1,30-1,50 \mathrm{~m})$ zwischen den beigegebenen Gefäßen gegen Bestattungen von erwachsenen Individuen. Vielleicht war also die Beigabe von Schwertern nicht nur erwachsenen Männern vorbehalten.

\section{Lanzenspitzen}

Der schlechte Erhaltungszustand fast aller eisernen Lanzenspitzen von Vergina macht den Versuch einer Formgliederung oder einer Einordnung in die von Snodgrass definierten Typen ${ }^{187}$ sinnlos. Auffällig ist, daß die Lanzen in Vergina stets einzeln mit ins Grab gegeben werden ${ }^{188}$. Ebenso wie die Schwerter gehören auch die Lanzen ausschließlich zu bescheidenen Inventaren, die erst in der Endstufe der Nekropole (Stufe IV) durch Toilettegerät (Pinzette) oder Schmuck (wenige Perlen) bereichert werden können (Abb. 39).

\section{Pfeilspirzen}

Die sechs Pfeilspitzen aus Malamas $\Gamma I$ sind zwar unterschiedlich groß, sie gehören aber alle dem gleichen Typ mit flachem, dreieckigem Blatt und mit Stiel an (Abb. 25,2-7) ${ }^{189}$. Eine einzelne Pfeilspitze dieser Form

184 So z. B. Andronikos 1969, $270 \mathrm{f}$.

185 Ebd. 44 Taf. 14.

$186 \mathrm{Vgl}$. auch Grab AZ I mit Saugkännchen und zierlichem Ringschmuck; gegenseitiger Abstand der Gefäße 0,65 m (ebd. 72). Entsprechend Lefkandi I, 203 f.; Vokotopoulou 1986, 337.

187 A.Snodgrass, Early Greek armour and weapons (1964) $120 \mathrm{ff}$;; siehe auch Vokotopoulou 1986, $300 \mathrm{ff}$.; Lefkandi I $254 \mathrm{f}$.

188 Im Gegensatz z. B. zu Attika oder Lefkandi, wo sie häufig in Schwertgräbern auftreten, oder zu Vitsa, wo entweder Schwert und Lanze oder zwei Lanzen ins Grab gelegt werden: Müller-Karpe 1962, 90 ff.; Abb. 8,6-8; 23; 28; B.Schlörb-Vierneisel, Athen. Mitt. 81, 1966, 7f. Beil. 12; Lefkandi I, 160f. Taf. 149-151; 175 f. Taf. 174-175; 195 f. Taf. 192; Vokotopoulou 1986, $302 \mathrm{f}$.

189 Zum Typ siehe Snodgrass (Anm. 187) 148; Lefkandi I, 256 f. ist aus dem protogeometrischen Grab 28 des Kerameikos bekannt ${ }^{100}$. Mit dem Befynd im Grab Malamas $\Gamma I$ von Vergina kann das spätprotogeometrische Grab 26 der Toumba-Nekropole von Lefkandi verglichen werden: eine Bestattung mit Eisenschwert und zehn Pfeilen, die zur Linken des Toten in einem Bündel (im Köchor?) niedergelegt waren ${ }^{191}$. Im Grab Malamas $\Gamma I$ sind es sechs gebündelte Pfeile nahe neben dem Schwert. Unklar bleibt hier, ob die eisernen Beschläge (Abb. 25,8-13) ursprünglich den Köcher oder die Schwertscheide zierten. Ins Grab mitgegeben wurden in Vergina ein oder drei Pfeile; nur im Grab Malamas $\Gamma I$ ist mit sechs Pfeilen die Anzahl verdoppelt. Es ist kaum vorstellbar, daß die tatsächliche Ausrüstung eines Bogenschützen - sei es für den Kampf oder die Jagd - je aus so wenigen Pfeilen bestand. Ihre Anzahl im Grab war also offensichtlich durch Beigabensitten reglementiert, die auch selektiv sein konnten. In der Nekropole von Vergina gibt es Grabinventare mit Pfeilen als einziger Waffe oder mit Pfeilen und Schwert; die Kombination von Lanze und Pfeilen ist nicht belegt (Abb. 39). Für eine Interpretation dieser Verteilung wäre erst die Funktion der Pfeile zu klären. Waren es Jagdwaffen, dann bedeutet ihr Vorkommen im Grab eher einen Repräsentationsanspruch, der wohl Schwertträgern, aber nicht Lanzenträgern zukam ${ }^{192}$. Gehörten sie aber vor allem zur Kriegsausrüstung, müßte man annehmen, daß es reine Bogenschützen gab und Schwertkämpfer (nicht aber Lanzenkrieger), die zusätzlich mit einem Bogen bewaffnet waren.

\section{Messer}

Die eisernen Messer aus den hier vorgestellten Grabhügeln lassen sich zwei Typen zuweisen: a) mit gewölbtem Rücken und gerader Schneide (Abb. 30,19); b) mit eingesatteltem Rücken und geschwungener Schneide (Abb. 25,14). Beide Formen sind bereits aus der Grabung Andronikos bekannt ${ }^{193}$. Vergleichbare Messer begegnen von Thessalien bis Attika in protogeometrischer bis früharchaischer Zeit ${ }^{19}$. Ebenso wie Kübler hat auch Andronikos die Eisenmesser insgesamt in die Kategorie Waffen eingeordnet ${ }^{195}$. Für die Messer mit eingesatteltem Rücken dürfte das schon wegen ihres kleinen Formats (Längen von $8-10,2 \mathrm{~cm}$ ) nicht zutreffen. Auch die Form der

190 Kübler 1943，27; 35 Taf. 38; Müller-Karpe 1962, 92 Abb. 10,7.

191 Lefkandi I, 182 f.; Taf. 162; 182-183; 202,c.

192 Auch im Kerameikos und in Lefkandi begegnen die Pfeile in Schwertgräbern (siehe Anm. 190-191).

193 z.B. Grab A II, ZIII, K VI, NVIII,-NXIV, TIX, TX, AAI; A $\triangle I I I$, AE $I V$, AZIII bzw. KI, A $\Gamma V I I I$, AHIV.

194 A.J.B. Wace u. M.S. Thompson, Annu. Brit. School Athens 18, 1911-12, $24 \mathrm{ff}$.; K. Kilian in: The Greek Renaissance of the Eighth Century B.C./Proceedings 2. Intern. Symposium Swedish Inst. Athens 1981 (1983) 138 Abb.7,1-5.9; Kübler 1943, 26 ff.; ders. 1954, 197 ff.; R.S. Young, Late Geometric graves and a Seventh Century well in the Agora. Hesperia Suppl. 2, 1939, 44 ff.; 93 f.; Lefkandi I, 257 f.

195 Kübler 1943, 28; Andronikos 1969, 266. 
geschwungenen Schncide wcist auf eine spezielle Funktion hin, wic z. B. Abhäuten oder Lederbearbeitung oder auch Rasicren. Als Hicbmesser und damit als Waffe ist wohl nur das über $40 \mathrm{~cm}$ lange Messer aus dem Pithosgrab 5 im Hügel $\mathrm{Z}$ zu klassifizieren ${ }^{196}$, während die übrigen Messer mit einer durchschnittlichen Länge von $20 \mathrm{~cm}$ für vielfältige Verwendung im täglichen Leben

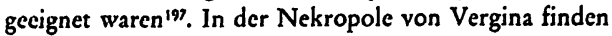
sich die kleinen Eisenmesser eincrscits in Gräbern mit Waffe (Schwert, Lanze, Pfeil), also in sicheren Männergräbern, andererseits in waffenlosen Gräbern, die aber nie typisch „weibliche" Trachtbestandteile enthalten (Abb. 39) ${ }^{198}$. Anders als in Attika und vermutlich auch in Thessalien scheinen sie demnach in Makedonien eine geschlcchtsspezifische, ausschließlich Männern zukommende Grabbeigabe gewesen zu sein ${ }^{199}$.

\section{Wetzstein}

In der Aufschüttung des Hügels $\Gamma$ (Feld Malamas) wurde nahe bei Grab VIII ein Wetzstein aus grauem Stein mit abgewetzten Kanten gefunden (Abb. 29,30). Ein zweiter Wetzstein aus Hügel $\Phi$ ist ebenfalls ein Streufund, der sich keiner Bestattung mehr zuweisen läß $\mathrm{St}^{200}$. Das einzige geschlossene Inventar mit Wetzstein von Vergina stammt aus Grab LXV $A \Gamma$, das außerdem eine Lanzenspitze, eine doppelkonische Perle und einen Krug enthielt ${ }^{201}$. Dieses Grab gehört der Stufe IV A der Nekropole an. Wetzsteine sind auch im südlichen Griechenland eine seltene Grabbeigabe. Man kennt bisher nur ein Exemplar aus dem spätgeometrischen Grab XXV von Tiryns und ein Fragment vom Brandplatz 8 der ToumbaNekropole von Lefkandi, das wahrscheinlich in frühgeometrische Zeit gehört ${ }^{202}$. Bei der Vorlage der Steingeräte aus der Siedlung von Kastanas werden kleine, gelochte wetzsteinförmige Anhänger und größere, meist nicht gelochte Wetzsteine unterschieden ${ }^{203}$. Danach wäre das Exemplar von Tiryns mit nur $6,5 \mathrm{~cm}$ Länge zu den Anhängern zu zählen. $\mathrm{Da}$ der Wetzstein aus dem Grabhügel $\Gamma$ (Feld Malamas) deutlich abgewetzte Kanten hat,

196 Ebd. 119; 266 f.; Abb. 104; Taf. 82; vergleichbar mit den Hiebmessern aus den geometrischen Gräbern von Halos: Kilian 1975 a, Taf. 21,3; 22,4.

197 So auch Lefkandi $I, 257 \mathrm{f}$.

198 Bei den Gräbern LXV $A B, \operatorname{LXV} A Z$ und LXV $A \Theta$ handelt es sich um beiseite geräumte Beigaben, so daß die Zusammengehörigkeit von Fibeln, Schmuck und Eisenmessern nicht gesichert ist. Für das Grab Lazaridis $V$ konnte die Fundstelle der zwei Messer und zwei Messergriffe nicht mehr festgestellt werden.

199 Vgl. Kübler 1954, 23; Wace u. Thompson (Anm. 194) 24ff.; ebenso in Vitsa: Vokotopoulou 1986, 297.

200 Andronikos 1969, 56; 132; 260 Taf. 106; ein weiteres Exemplar aus Perivolaki: Heurtley (Anm. 39) Abb. 103,d.

201 Petsas 1961-62, 280 Taf. 138.

202 Verdelis (Anm. 75) 42 ff.; Lefkandi I, 195; Taf.192, 8:6; 237 ,f.

${ }^{203}$ Hochstetter (Anm. 92) $53 \mathrm{f}$. ist trotz Durchbohrung und geringer Stärke $(0,6 \mathrm{~cm})$ eine tatsächliche Verwendung als Gerät sicher.

\section{Ergebnisse}

Als wohl wichtigstes Ergebnis der Rettungsgrabung von 1970 in der Hügelnekropole von Vergina kann der gesicherte Nachweis einer Doppelbestattung im Grab Malamas $\Gamma I$ gelten. Mindestens zwei Individuen waren in den Gräbern LXV $A Z$ sowie LXV $A B$ und LXV $A O$ bestattet; es handelt sich dort aber um eine sukzessive Belegung, wie die beiseitegeräumten Beigaben zeigen ${ }^{204}$. "Männliche" und "weibliche" Beigaben enthalten auch die Gräber LXV BA und LXVE. Bei ihrer Verteilung im Grab bleiben jedoch Zweifel, ob die Bestattung von Mann und Frau gleichzeitig erfolgte ${ }^{205}$. Einziges Indiz für eine Doppelbestattung im Pithos 1 des Hügels $\Phi$ ist die Beigabe von zwei Krügen zusammen mit zwei Schalen ${ }^{206}$. Nicht eindeutig ist schließlich der Befund im Grab T I: Dicht neben einer anscheinend ungestörten Bestattung mit Schwert und Keramik lagen Spiralröllchen und Tutulus vom Kopfschmuck einer Frau, einige Perlen und eine Brillenfibel, während die vermutlich zugehörige zweite Brillenfibel $2 \mathrm{~m}$ entfernt war ${ }^{207}$. Diese beiden Bestattungen sind wahrscheinlich, wie schon Andronikos annahm ${ }^{208}$, in einer zeitlichen Abfolge zu sehen, d.h. bei Anlage des Schwertgrabes wurde das Frauengrab teilweise gestört. Die im Grab Malamas $\Gamma I$ angetroffene Fundlage des Inventars dagegen erklärt sich nur aus der gleichzeitigen Grablege zweier Individuen in paralleler Ausrichtung und zwar einer reich ausgestatteten Frau zur Linken eines Mannes mit Schwert und Lanze. Doppelbestattungen sind auch im übrigen Griechenland während der protogeometrischen und geometrischen Zeit selten. In Lefkandi enthält das Grab 14 der ToumbaNekropole zwei Amphoren mit Leichenbrand; nach den Beigaben sind es die Bestattungen von Mann und Frau ${ }^{209}$. Im Grab 45 der Palia Perivolia-Nekropole dagegen sind beide Tote mit Fibeln ausgestattet, so daß eine archäologische Geschlechtsbestimmung nicht möglich ist ${ }^{210}$. Der Brandplatz XIV von Halos mit zwei Schwertern und zwei Lanzenspitzen kann als Doppelbestattung zweier

\footnotetext{
${ }^{204}$ Petsas 1961-62, 278 ff.; Abb. 56; Taf. 138,b; 139,b. ${ }^{205}$ Ebd. 287 Taf. 144,b; Petsas 1963, 218 f. Abb. 3.

206 Andronikos 1969, $55 \mathrm{f}$.

207 Ebd. 47 f. Abb. 13.

208 Ebd. 48.

209 Lefkandi I, $175 \mathrm{f}$.; Taf. 159; 174-175.

210 Ebd. 159; Taf. 123; 149; die Größe des Grabes und die Lage der Skelette nebeneinander sind hier Indizien für die gleichzeitige Bestattung der beiden Toten. Im Gegensatz dazu enthalten die Gräber XXV und XXVIII von Tiryns eine primäre und eine sekundäre Bestattung, da jeweils eines der Skelette deutlich beiseite geräumt ist (Verdelis [Anm. 75] $10 \mathrm{ff}$.; $42 \mathrm{ff}$.).
} 
waffentragender Männer interpretiert werden ${ }^{211}$, aber weder die Gleichzeitigkeit noch die Aufteilung in zwei gleichartige Waffenausstattungen ist durch den Grabungsbefund erwiesen. Einige Beispiele für Doppelbestattungen Erwachsener finden sich jedoch in den Grabhügeln von Barç und $\mathrm{Kuç} \mathrm{i} \mathrm{Zi}$ in Albanien ${ }^{212}$, während in der Nekropole von Vitsa nur im Grab 84 eine Frau und ein Kind (Murter mit Kind?) zusammen begraben sind ${ }^{213}$. In Analogie zu ethnologisch bezeugten Sitten werden auch prähistorische Doppelbestattungen von Mann und Frau häufig als "Witwenopfer" erklärt214. Bei der Seltenheit solcher Gräber in der Nekropole von Vergina müßte es sich allerdings um einen nur für sehr spezielle Sonderfälle gültigen Brauch handeln. Mögen uns auch konkreter Anlaß und Motive unzugänglich bleiben, die Doppelbestattung im Grab Malamas $\Gamma I$ bedeutet, daß es innerhalb der sozialen Gruppierung für Frauen möglich war, einen Status zu erreichen, der dem der waffentragenden Männer gleich kam (siehe auch unten S.136).

Die Untersuchung des Grabhügels Malamas $\Gamma$ ergab zudem einige für die Grabsitten von Vergina aufschlußreiche Detailbeobachtungen. Im Grab $\Gamma I$ liegen die zum Kopfschmuck gehörigen Spiralröllchen, Tutuli und Brillenfibeln auf einer $2-3 \mathrm{~cm}$ starken Erdschicht, die zum Halsring hin ausdünnt (Abb.21). Nach den publizierten Grabungsphotos wurde die gleiche Situation auch in den Gräbern $\mathrm{O} I, \mathrm{Y} I, \mathrm{~A} \triangle I, \mathrm{AE} V$ und $\mathrm{AH} I I$ angetroffen 215 . Die Sohle der Grabgrube scheint also am einen Ende so zugerichtet worden zu sein, daß der Kopf der Toten auf einer kissenartigen Erhöhung zu liegen kam $^{216}$. Deutlich ist im Grab Malamas $\Gamma I$ auch die seitliche Verlagerung des Kopfschmucks. Da Spiralröllchen und Tutulus dabei im ursprünglichen Verband blieben, sind sie vermutlich zusammen mit dem Schädel zur Seite gerutscht. Eine derartige Verschiebung ist kaum möglich, wenn der Tote unmittelbar mit Erde bedeckt war. Noch auffälligere Verlagerungen von Beigaben, z. B. im Grab AZ VII, hat bereits Andronikos als Hinweis darauf gewertet, daß es zwischen dem Toten und der Auffüllung der Grabgrube einen freien $Z$ wischenraum gegeben haben muß217. Das Fehlen jeglicher Skelettreste in den prähistorischen Gräbern von Vergina hat Andronikos auf die Bodenbeschaffenheit zurückgeführt218. Beobachtungen in den Nekropolen von Lefkandi mit Gräbern, die zwar keine Skelettreste, aber als Beigaben Trachtbestandteile in annähernd

211 Wace u. Thompson (Anm. 194) 17; $25 \mathrm{f}$.

212 Barç, Hügel I,38: $\mathrm{Zh}$. Andrea, Kultura ilire e tumave ne pellgun e Korçes (1985) 26 Abb.23. - Kuç i Zi, Hügel 1,19.32: dies. (Anm. 163) 173 ff. Abb. 2.

${ }^{213}$ Vokotopoulou 1986, $335 \mathrm{f}$.

214 R. Peroni in: ders. (Hrsg.), Necropoli e usi funerari nell' età del ferro (1981) $300 \mathrm{ff}$.

215 Andronikos 1969, Taf. 13; 16; 23; 25; 29.

216 Das Unterlegen eines Steins unter den Kopf ist z. B. aus der Nekropole von Vitsa bekannt: Vokotopoulou 1986, 335.

217 Andronikos 1969, 162.

218 Ebd. 163. realer Position enthalten, waren für P. Themelis Anlaß, auch für die Gräber von Vergina eine Interpretation als ${ }^{\prime} K_{\text {Kenotaphe }} e^{\alpha u}$ erwägen ${ }^{219}$. Das Vorkommen menschlicher Zähne läßt sich allerdings in Gräbern ohne Tote nur schwer erklären ${ }^{20}$. Vollends unmöglich wäre für Kenotaphe ein Befund wie Malamas $\Gamma I I$, wo die Zahnreihen von Ober- und Unterkiefer in ihrer natürlichen Anordnung lagen (Abb. 27).

Die übliche Waffenbeigabe in der Nekropole von Vergina sind Schwert und Lanze, die aber in der Regel nicht zusammen ins Grab gegeben werden (Abb. 39). Ist die tatsächliche Bewaffnung der allein bestimmende Faktor für die Ausstattung im Grab, so bedeutet diese Ausschließlichkeit, daß die Streitmacht von Vergina aus den zwei Truppen der Schwert- und der Lanzenkämpfer bestand ${ }^{221}$. Wird aber mit der durch Grabsitten geregelten Auswahl der Beigaben umfassender die Rolle des Toten in der sozialen Organisation charakterisiert, dann zeigen die Inventare in Männergräbern eine klare Einteilung in vier Gruppierungen: 1. mit Schwert, 2. mit Lanze, 3. mit kleinem Messer und 4. nur mit Keramik ${ }^{222}$. Eine weitere Differenzierung innerhalb der Gruppen 1 und 2 besteht lediglich im Vorkommen bzw. Fehlen des Messers; als einziges Schmuckstück treten gelegentlich Fingerringe auf und in der Endphase der Nekropole einzelne Perlen. Bemerkenswert ist insgesamt das Fehlen von Trachtzubehör, Schmuck, Geräten und jeglicher Art von Luxusoder Prestigeobjekten. Die Waffe allein war es also, die den Status eines Mannes kennzeichnete, wobei angenommen werden darf, daß sich in der Wahl von Schwert bzw. Lanze ein Qualitäts- oder Rangunterschied manifestierte. Die kleinen Messer der dritten Inventargruppe waren wohl keine Waffen. Ihre statusrelevante Bedeutung könnte auf einer ritualisierten Funktion beruhen, wenn sie z. B. bei der Teilnahme an Festmählern als Tafelbesteck dienten.

Auch den Frauen sind weder Gerät $t^{23}$ noch Luxusgegenstände mit ins Grab gegeben. Ihre Ausstattung besteht im wesentlichen aus Trachtzubehör und Schmuck, in dessen vielfältig variablen Kombinationen sich keine deutlichen Gruppierungen abzeichnen (Abb. 36-38). Es kann vermutet werden, daß sich in

219 P. Themelis in: Lefkandi I, 213.

220 z. B. in den Gräbern A II, $\Delta I I, \mathrm{E} V I I I, \mathrm{~K} I I I, \mathrm{~N} I V, \mathrm{~N} V$, $\mathrm{N} I X, \mathrm{~N} X I, \Xi I, \Xi I I I, \mathrm{P} I I, \mathrm{P} V, \mathrm{P} V I, \mathrm{P} V I I I, \mathrm{~T} I, \mathrm{Y} I I I$, $\Phi I I I, \mathrm{AE} I X$.

221 So urteilt z. B. Andronikos 1969, 270f. Eine derartige militärische Gliederung wäre jedoch für die anderen griechischen Landschaften, wo öfters das Schwert zusammen mit der Lanze zur Grabausstattung gehört, nicht anzunehmen.

$22 z$ Da sich bei der Keramik keine Unterscheidung von Gefäßformen in Männer- und Frauengräbern nachweisen läßt, bleibt die Annahme hypothetisch, daß sich die nur Keramik enthaltenden Gräber auf Männer und Frauen verteilen.

223 Ausnahme sind vielleicht Spinnwirtel, die Andronikos aus den Gräbern EII, $\Xi I V$, A $\Gamma X V I I, \mathrm{AZ} V I I, \mathrm{AH} V$ und AH IX nennt (Andronikos 1969, 260). 
bestimmten Formen des Kopfschmucks und in der Zahl bzw. Größc von Fibcln der Stand der verheirateten Frau oder dic Zugehörigkeit zu Altersgruppen ausdrückt; cs fellt jedoch an anthropologischen Daten für eine Überprüfung. In augenfälligem Kontrast zu den Männerbestattungen ist das Inventar der Frauengräber durch die mchr oder weniger reichhaltige Ansammlung von Schmuck eine Demonstration des Reichtums, in der sich in hohem Maße die wirtschaftliche Lage spiegelt. Andererseits sind es allein die Frauengräber, in denen sich so ausgesprochene Statussymbole wie die Drillingsbeile finden. Es handelt sich bei ihnen wohl um eine spezialisierte Ritualausrüstung aus dem kultischen oder profanen Bereich. Ihr ausschließliches Vorkommen in Gräbern mit besonders rcichem Schmuck weist darauf hin, daß der durch sie angezeigte Status nur Angehörigen der privilegierten Schicht zukommen konnte. Innerhalb der Sozialorganisation hatten solche Frauen, wie die Doppelbestattung im Grab Malamas $\Gamma$ I zeigt, den gleichen Rang wie schwerttragende Männer ${ }^{224}$.

Geringere Dimensionen der Grabgrube und zierlicher Schmuck sprechen dafür, daß in den Grabhügeln von Vergina außer erwachsenen Frauen und Männern auch Kinder bestattet wurden ${ }^{225}$. In ihren Beigaben unterscheiden sie sich nicht von den Erwachsenen. Selbst Schwerter begegnen in vermutlichen Kindergräbern, nur die sehr reiche Tracht- und Schmuckausstattung scheint erwachsenen Personen vorbehalten. Eine Erklärung dafür liegt in der Annahme, die soziale Stellung, die sich im Anspruch auf Grabbeigaben ausdrückt, wäre nicht vom Alter abhängig und erworben, sondern erblich. Es sollte aber auch mit der Möglichkeit gerechnet werden, daß das Recht auf Bestattung im Grabhügel erst mit einer bestimmten Altersstufe erlangt wurde, d.h. zumindest im bisher erforschten Nekropolenteil könnten nur Jugendliche und Erwachsene liegen, während für Kinder und Kleinkinder ein gesondertes Areal bestimmt war ${ }^{226}$. $\mathrm{Da}$ in Vergina die einzelnen Grabhügel meist über mehrere Zeitphasen hin belegt wurden und zwar mit Bestattungen von Frauen, Männern und Kindern, liegt es nahe, in ihnen Familiengrabstätten zu sehen ${ }^{227}$. Bei einer Geschlechtsbestimmung nach archäologischen Kriterien ergeben sich für einige Hügel annähernd ausgewogene Anteile von Männern und Frauen, in anderen Hügeln dagegen ist die Zahlendiskrepanz für einen „natürlichen“ Familienverband zu groß ${ }^{228}$. Als besonders eklatante Beispiele seien der Hügel $\mathrm{T}$, mit acht Männern und zwei

224 Innerhalb der Nekropole fällt auf, daß Gräber mit Drillingsbeilen nur in den Hügeln vorkommen, in denen es auch Schwertgräber gibt - einzige Ausnahme der Hügel AH.

225 So bereits Andronikos 1969, 152.

226 Aus Grab Malamas B II stammt jedoch der Milchzahn eines 3- bis 5jährigen Kindes: siehe Anhang 2.

227 z. B. Andronikos 1969, $280 \mathrm{f}$.

228 Vgl. die Tabelle mit der Verteilung von Männer- und Frauengräbern in den Hügeln: Ebd. 153.
Frauen, und der Hügel $\mathrm{N}$ genannt, dessen 19 Gräber sich auf drei Männer, 13 Frauen und drei Unbestimmbare verteilen. In dieser Nekropolenstruktur spiegelt sich wohl cine Sozialorganisation wider, in der die Grablege nicht in jedem Fall durch die Familienbindung bestimmt war. Grabhügel mit übcrwiegenden Männerbestattungen könnten auf militärische Verbände in der Art von Gefolgschaften hinweisen oder auf Männerbünde, wie sie in Makedonien aus historischer Zeit bekannt sind ${ }^{22 \%}$. Exklusive Bestattungsplätze für Männer haben notwendig entsprechende Grabgemeinschaften für Frauen zur Folge. Da Männer- und Frauengrabhügel nebeneinander in der gleichen Nekropole angelegt sind, bedeutet diese Trennung keine soziale Deklassierung, sondern wohl eher eine gleichrangige Stellung der Frauen bei den Bewohnern des früheisenzeitlichen Vergina.

Die Ausdehnung der Nekropole und die Zahl der Grabhügel sind noch nicht genau festgestell ${ }^{230}$. Von einer früheisenzeitlichen Siedlung, die Andronikos auf den Terrassen unterhalb des hellenistischen Palastes nachweisen konnte, kennt man einstweilen nur geringe Spuren ${ }^{231}$. P. Phaklaris hat kürzlich auf die problematische Beziehung zwischen bescheidener Siedlung und ausgedehnter Nekropole hingewiesen und die Möglichkeit in Erwägung gezogen, daß Vergina der zentrale Bestattungsplatz einer größeren Region und deren halbnomadischer Bevölkerung war ${ }^{232}$. Bei unseren gegenwärtigen Kenntnissen über die frühe Eisenzeit in Makedonien sind Aussagen zur wirtschaftlichen Grundlage und zum Siedlungsbild kaum möglich ${ }^{233}$. Obwohl die intensivierte Forschung im Gebiet des Olymp mehrere Hügelnekropolen nachweisen konnte ${ }^{23+}$, sind die zugehörigen Siedlungen noch nicht lokalisiert und andererseits müssen die Friedhöfe der großflächig ausgegrabenen Siedlungen von Kastanas und Assiros erst noch entdeckt werden ${ }^{235}$. Es ist zu hoffen, daß zukünftige Ausgrabungen Vergleichsma-

229 So bereits K. Kilian in: 24. Convegno di studi sulla Magna Graecia, Tarent 1984 (im Druck).

230 Andronikos 1969, 2 schätzt eine Zahl von 300 Hügeln. Ein 1969 im Grundstück N. Goultidis zerstörtes Grab (Rhomiopoulou [Anm. 80] 13f.) und eine früheisenzeitliche Bestattung unter der Aufschüttung der hellenistischen Großen Toumba (P. Phaklaris in: Amitos/Festschrift M. Andronikos [1987] $923 \mathrm{ff}$.) lassen vermuten, daß sich die Nekropole weiter nach Süden bis in den Bereich des heutigen Dorfes erstreckte.

231 Andronikos 1969, $3 \mathrm{f}$.

232 Siehe Anm. 230.

${ }^{233} \mathrm{Zu}$ Wirtschaftsformen vgl. B. Hänsel, Jahrb. RGZM 26, 1979, 167 ff.; H.J. Kroll, Kastanas / Die Pflanzenfunde. Prähist. Arch. Südosteuropa 2 (1983); C. Becker, Kastanas / Die Tierknochenfunde. Prähist. Arch. Südosteuropa 5 (1986).

23+ Erster Überblick über die Ergebnisse durch E. Poulaki-Pandermali in: Amitos / Festschrift M. Andronikos (1987) 705 ff; siehe auch Ancient Macedonia (Anm. 96) 23 mit Nr. 56-63. 82-93.

235 Hänsel (Anm. 233); H. W. Catling, Arch. Reports 1975-76, $19 \mathrm{ff} . ; 1977-78,44$ ff.; $1980-81,30 \mathrm{ff}$. 
terial erbringen, aus dem die Situation der Nekropole von Vergina verständlicher wird.

\section{Katalog}

Die Keramik ist, soweit nicht anders erwähnt, handgefertigt. Ofl. = Oberfläche.

\section{Feld Lazaridis, Grabhügel}

\section{Grab I}

a. Krug mit ausgeschnittenem Nacken, Ofl. graubraun, gefleckt, Bruch dunkel rotbraun, feiner Ton, geglättet (auf dem Hals senkrechte Glättspuren), H. $17 \mathrm{~cm}$ (Inv. Nr. 1108). Abb. 4,1; 40,e.

b. Kantharos, Schulter gerieft, Ofl. grau bis graubraun, Bruch grau, geglättet. Dm. $14 \mathrm{~cm}, \mathrm{H} .13,5 \mathrm{~cm}$ (Inv. Nr. 1107). Abb. 4,$2 ; 40$,b.

c. Schwert, Eisen, auf Griffzunge und Schulter Spuren des Griffbelags aus Holz (?), L. $84 \mathrm{~cm}$ (Inv. Nr. 823). Abb.6,1; $43, \mathrm{~b}$.

d. Kleiner Ring, Bronze, Dm. 2,4 cm (Inv. Nr. 824). Abb. 6,2; $41, \mathrm{a}$.

e. Kleiner Ring, Bronze, Dm. 2,1 cm (Inv. Nr. 824). Abb. 6,3; $41, \mathrm{~b}$.

f-g. Zwei Tutuli (nicht nachweisbar).

Grab II

a. Einhenkeliger Becher, Schulter schräg gerieft, Ofl. grau bis graubraun, Bruch graubraun, geglättet. $\mathrm{H} .11 \mathrm{~cm}$ (Inv. Nr. 1106). Abb. 4,5; 40,d.

Grab III

a. Knopfhenkelschale, Ofl. rotbraun, geglättet, $\mathrm{Dm} .13,5 \mathrm{~cm}$, H. 10,5 cm (Inv. Nr. 1100). Abb. 4,6; 40,f.

b. Knopfhenkelschale, beide Knopfaufsätze antik gebrochen, Ofl. und Bruch hell lederfarben, geglättet, Dm. $18-18,9 \mathrm{~cm}$, H. 13,5 cm (Inv. Nr. 1101). Abb.4,8; 40,c.

\section{Grab IV}

a. Amphoriskos, Teile von Hals und Schulter fehlen, Ofl. rotbraun, Bruch rotbraun mit grauem Kern, geglärtet, Dm. $10,5 \mathrm{~cm}$, H. 18,5 cm (Inv. Nr. 1110). Abb. 4,7.

b. Unterer Teil eines Kruges, Ofl. und Bruch rotbraun, geglättet, H. noch 8,5 cm (Inv. Nr.1111). Abb. 4,4.

c. Brillenfibel, Bronze, L. 8,8 cm (Inv. Nr. 831). Abb.6,8.

d. Fingerring mit gegenständigen Spiralenden, Bronze, Dm. $1,7 \mathrm{~cm}$ (Inv. Nr. 832). Abb. 6,7.

Grab V

a. Amphoriskos, Ofl. graubraun bis schwarz, Bruch braun, grob gemagert, geglärtet (auf dem Hals senkrechte Glättspuren), Dm. 8,7-9,2 cm, H. 14,8 cm (Inv. Nr.1103). Abb. 5,$4 ; 42$,d.

b-c. Zwei Brillenfibeln, Bronze, L. $13 \mathrm{~cm}$ und $13,4 \mathrm{~cm}$ (Inv. Nr. 825-826). Abb.7,1-2; 41,c-d.

d. Tutulus mit Öse, Bronze, Dm. $3 \mathrm{~cm}$ (Inv. Nr. 827). Abb. 7,7; 41,f.

e. Kleiner Ring, an einer Stelle ausgewetzt, Bronze, Dm. $2,8 \mathrm{~cm}$ (Inv. Nr. 830). Abb. 7,10; 41,e.

f. Zwei vollstāndige Spiralröllchen, Bronze, L. $7,6 \mathrm{~cm}$ und $7,1 \mathrm{~cm}$;

zwei fragmentierte Spiralröllchen, Bronze, L. noch $5,5 \mathrm{~cm}$ und 4,7 cm (Inv. Nr. 828). Abb. 7,11-14.

g. Zwei Tutuli mit Öse, Bronze, Dm. 1,7 cm und 1,6 cm (Inv. Nr. 827). Abb. 7,8-9; 41,g.

h. Messer, Eisen, Spitze gebrochen, Spuren vom Griff aus Holz (?), L. noch 15,5 cm (Inv. Nr. 829). Abb. 7,3; 41, h.

i. Messer, Eisen, Spitze gebrochen, Reste vom Griff aus Holz (?), L. noch 11,4 cm (Inv. Nr. 829). Abb. 7,4; 41,i. j-k. Fragmente von kleinen Messern mit gebogenem Rücken, Eisen, Reste vom Griff aus Holz (?), L. noch $4,3 \mathrm{~cm}$ und $3,3 \mathrm{~cm}$ (Inv. Nr. 830). Abb. 7,5-6.

Grab VIa

c. Skyphos, Ofl. gelblich weiß, Bruch rotbraun, Firnis rotbraun, dick, Henkelinnenseite tongrundig, Drehscheibenarbeit, Dm. 9,2 cm, H. 8,1 cm (Inv. Nr. 1104). Abb. 5,3; 42,b.

d. Schwert, Eisen, auf Griffzunge und Schulter Abdruck des Griffbelags, L. $70 \mathrm{~cm}$ (Inv. Nr. 822). Abb. 6,4; 43,a.

e. Fingerring mit gegenständigen Endspiralen, Bronze, Dm. $2,2 \mathrm{~cm}$ (o. Nr.). Abb. 6,5.

f. Kleiner Ring, Bronze, Dm. 2,5 cm (o. Nr.). Abb.6,6.

\section{Grab VI $b$}

a. Knopfhenkelschale, Ofl. und Bruch rotbraun, geglättet, Dm. 18-19 cm, H. 11,5 cm (Inv. Nr. 1102). Abb. 5,1; 42,c.

b. Krug mit ausgeschnittenem Nacken, Ofl. grau mit lederfarbenen Flecken, Bruch grau bis lederfarben, geglättet, $H$. 16,6 cm (Inv. Nr. 1105). Abb. 5,2; 42,a.

\section{Grab VII}

a. Unterer Teil eines Amphoriskos und zwei nicht anpassende Scherben vom Kegelhals, Ofl. und Bruch rotbraun, grob gemagert, geglättet, $H$. noch $11 \mathrm{~cm}$ (Inv. Nr.1112). Abb. 4,3.

b. Krug (nicht nachweisbar).

Einzelfund aus dem Hügel

- Kantharos, Schulter gerieft, Ofl. grau, Bruch rotbraun, feine Kalkmagerung, Ofl. verstrichen, Dm. 19,5 cm (Inv. Nr. 1109). Abb. 5,5; 40,a.

Feld Malamas, Grabhügel A

Grab I

a. Schale mit zwei randständigen Horizontalhenkeln, Ofl. und Bruch rotbraun, geglättet, $H .8 \mathrm{~cm}$ (Inv. Nr.1114). Abb. 5,7.

b. Einhenkeliger Becher, Ofl. und Bruch rotbraun, geglättet, Dm. 11,2-11,5 cm (Inv. Nr.1113). Abb. 5,6.

c. Krug mit ausgeschnittenem Nacken, unvollständig, Ofl. braun mit schwarzen und roten Brennflecken, Bruch grau, gut geglättet, H. 17,7 cm (Inv. Nr. 1115). Abb. 5,8.

Grab II

a. Schwert, Eisen, Griffzunge gebrochen, auf Schulter Abdruck des Griffbelags aus $\mathrm{Holz}$ (?), L. noch $39,8 \mathrm{~cm}$ (Inv. Nr. 833). Abb. 7,15.

Grab II a

a. Brillenfibel, Bronze, L. $4,6 \mathrm{~cm}$ (o. Nr.). Abb.7,17.

$\mathrm{b}-\mathrm{c}$. Zwei kleine Ringe, Bronze, innen und außen anhaftende Gewebereste, Dm. 2,3 cm und 2,4 cm (o. Nr.). Abb.7,16.

d. Zwei Tutuli mit Öse, Bronze, Dm. $1,5 \mathrm{~cm}$; ein Tutulus mit Dorn, Bronze, Dm. 0,7 cm (o. Nr.). Abb. 7,18-20.

\section{Feld Malamas, Grabhügel B}

Grab I

4. Unterer Teil eines Kruges (?), Ofl. und Bruch grau, geglättet, Bodendm. 6,2 cm (Inv. Nr. 1120); dabei zwei Gefäßböden, Ofl. rotbraun; zwei sehr kleine Scherben von Drehscheibenkeramik, gefirnißt (o. Nr.).

Abb. $12,3$.

2.13 Zwei Brillenfibeln, Bronze, L. $11,3 \mathrm{~cm}$ und $11,4 \mathrm{~cm}$ (Inv. Nr. 836-837). Abb. 7,21-22.

14. Zwei Lockenringe, Gold, Dm. $2,5 \mathrm{~cm}$ und $2,3 \mathrm{~cm}$ (Inv. Nr. 834). Abb. 7,23-24.

3.15 Mehrere Spiralröllchen (nicht nachweisbar). 

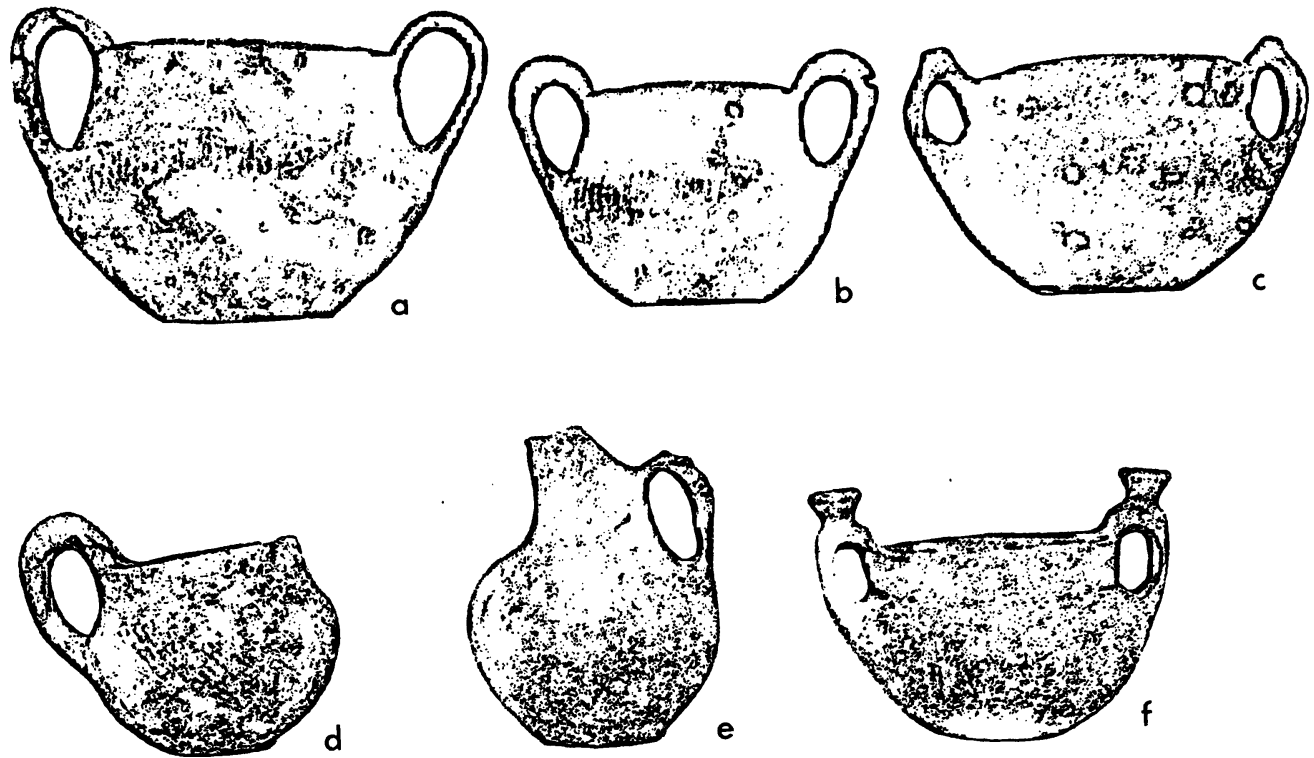

Abb. 40. Vergina. Feld Lazaridis, Grabhügel. a Streufund; b.e Grab I; c.f Grab III; d. Grab II

Grab Ia

7. Einhenkeliger Becher, Ofl. weißlich gelb, Firnis dunkelbraun, Henkelinnenseite tongrundig, Drehscheibenarbeit, Dm. 9-9,5 cm, H. $7 \mathrm{~cm}$ (Inv. Nr. 1123). Abb. 12,6.

8. Krug mit ausgeschnittenem Nacken, Ausguß gebrochen, Ofl. lederfarben, Bruch braun, gut geglättet, seifig, H. noch $13,6 \mathrm{~cm}$ (Inv. Nr. 1126). Abb. 12,5 .

Grab Ib

5. Schale mit Knopfhenkeln, Ofl. und Bruch grau bis rotbraun, geglättet, Dm. $15,5-16 \mathrm{~cm}, H .10,3 \mathrm{~cm}$ (Inv. Nr. 1134). Abb. 12,1.

6. Krug mit ausgeschnittenem Nacken, Ofl. braun, Bruch rotbraun, feine Glimmermagerung, geglättet, $H .18 \mathrm{~cm}$ (Inv. Nr.1122). Abb. 12,2.

Grab II

2. Schale mit Knopfhenkeln, ein Knopfaufsatz antik gebrochen, Ofl. und Bruch rotbraun, geglättet, $D \mathrm{~m} .12 \mathrm{~cm}, \mathrm{H}$. $8,4 \mathrm{~cm}$ (Inv. Nr. 1125). Abb. 12,12; 44,f.

3. Skyphos, Ofl. gelblich weiß, Firnis dunkelbraun, Henkelinnenseite tongrundig, feine Glimmermagerung, Drehscheibenarbeit, Dm. 13,2-14,4 cm (Inv. Nr. 1129). Abb.12,8; $44, c$.

4. Krug mit ausgeschnittenem Nacken, Ofl. und Bruch grau, geglättet, H. 11,5 cm (Inv. Nr. 1128). Abb. 12,11; 44,d.

7. Halsring, Bronze, tordiert, Dm. $11 \mathrm{~cm}$ (Inv. Nr. 840). Abb. 17,1.

8. Armspirale, Bronze, Dm. $3,3 \mathrm{~cm}$ (Inv. Nr. 839). Abb. 17,2 .

9. Tutulus mit Öse, Bronze, Dm. 1,7 cm (o. Nr.). Abb. 17,3.

10. Kleines Spiralröllchen, Bronze, L. $2,7 \mathrm{~cm}$ (o. Nr.). Abb. 17,4.

11. Drei Perlen, Sard (?) (o. Nr.). Abb. 17,5-7.

- Ein menschlicher Backenzahn (siehe Anhang 2).

Zugehörigkeit zum Grab II fraglich:

1. Krug mit ausgeschnittenem Nacken, Ofl. graubraun mit rotbraunen Brennflecken, Bruch grau bis graubraun, geglättet, H. noch 17,8 cm (Inv. Nr.1127). Abb. 12,10; 44,a.
5. Krug mit ausgeschnittenem Nacken, Ofl. und Bruch rotbraun, Ofl. verstrichen, H. $14,6 \mathrm{~cm}$ (Inv. Nr. 1124). Abb. 12,7; 44,e.

6. Amphoriskos, Ofl. braun mit rotbraunen und grauen Brennflecken, geglättet, Henkel im Brand aufgeplatzt, $H$. 14,3 cm (Inv. Nr. 1130). Abb. 12,9; 44,b.

Grab III

1. Zwei Tutuli, Bronze, Dm. $3,7 \mathrm{~cm}$ (Inv. Nr. 844); zwei Fragmente von Spiralröllchen, Bronze, L. noch $5,5 \mathrm{~cm}$ und $3 \mathrm{~cm}$ (Inv. Nr. 844).

Abb. 17,11-13.

2. Massiver Armring, Bronze, Dm. $9,5 \mathrm{~cm}$ (Inv. Nr. 843). Abb. 17,10.

3. Armspirale, Bronze, ein Ende antik gebrochen, Dm. unten 6,6 cm (Inv. Nr. 841). Abb. 17,8.

4. Lockenring, Gold (nicht nachweisbar).

5. Brillenfibel, Bronze, L. $12,5 \mathrm{~cm}$ (Inv. Nr. 842). Abb. $17,9$.

Grab IV

1. Einhenkeliger Becher, Ofl. lederfarben, geglättet, Dm. $12,5-13 \mathrm{~cm}$, H. 8,2 cm (Inv. Nr. 1135). Abb. 13,1.

3. Fragmente einer Knopfhenkelschale, Ofl. und Bruch ziegelfarben, geglättet, Dm. $15 \mathrm{~cm}, \mathrm{H} .10,3 \mathrm{~cm}$ (Inv. Nr. 1136). Abb. 13,9.

2. Messer, Eisen, Reste vom Griff aus $\mathrm{Holz}$ (?), L. $10 \mathrm{~cm}$ (Inv. Nr. 844 a). Abb. 18,1.

Grab V

1. Krug mit ausgeschnittenem Nacken, Ofl. grau bis graubraun, Bruch hellgrau, geglättet, H. 16,4 cm (Inv. Nr. 1116). Abb. 16,7.

2. Knopfhenkelschale, Ofl. lederfarben, geglättet, Dm. 13,5-14 cm, H. 8,5 cm (Inv. Nr. 1117). Abb. 16,8.

3. Hälfte einer Schale mit vier Henkeln, Ofl. hell lederfarben, Bruch grau, geglättet, Dm. $18,3 \mathrm{~cm}$ (Inv. Nr.1118). Abb. 16,5. 
Grab VI

5. Krug mit ausgeschnittenem Nacken, Ofl. grau mit rotbraunen Brennflecken, Bruch grau, geglättet (auf dem Hals senkrechte Glättspuren, auf dem Gefäßkörper waagerechte), H. 18,5 cm (Inv. Nr.1131). Abb. 13,11.

4. Schale mit zwei Henkeln und zwei Grifflappen, Ofl. beige, grob gemagert, geglättet, H. mit Henkeln $10,8 \mathrm{~cm}, \mathrm{Dm}$. $18 \mathrm{~cm}$ (Inv. Nr. 1137). Abb. 13,3.

10. Knopfhenkelschale, fragmentiert, Ofl. grau mit dünner, rotbrauner Brennhaut, Bruch nach außen rotbraun, nach innen grau, geglättet, $H$. noch $8,2 \mathrm{~cm}$ (Inv. Nr.1139). Abb. 13,5 .

11-12. Zwei Schildringe, Gold (Inv. Nr. 849). Abb. 17,18-19.

8-9. Zwei Brillenfibeln, Bronze, L. $8,7 \mathrm{~cm}$ und $5,4 \mathrm{~cm}$ (Inv. Nr. 848-847). Abb. 17,14.17.

6-7. Zwei massive Armringe, Bronze, Dm. $9,5 \mathrm{~cm}$ und $9,2 \mathrm{~cm}$ (Inv. Nr. 845-846). Abb. 17,15-16.

- Sechs menschliche Zähne eines Individuums (siehe Anhang 2).

Fundgruppe VIa

13. Saugkännchen, Ofl. rotbraun mit grauen Brennflecken, Bruch rotbraun, geglättet, H. $11,3 \mathrm{~cm}$ (Inv. Nr.1138). Abb. 13,2.

14. Krug mit ausgeschnittenem Nacken, Ofl. lederfarben, Bruch braun, geglättet, H. $11 \mathrm{~cm}$ (Inv. Nr. 1140). Abb. 13,8.

15. Drei kleine Ringe, gegossen, Bronze, Dm. $2,8 \mathrm{~cm}$ und $2,8 \mathrm{~cm}$ und $2,9 \mathrm{~cm}$;

aus Draht zusammengebogener Ring, Bronze, Dm. $2,4 \mathrm{~cm}$ (Inv. Nr. 852).

Abb. 17,20-23.

16. Perle, Sard (?) (o. Nr.). Abb. 17,24.

Fundgruppe VIb

1. Schale mit zwei Henkeln und zwei Grifflappen, Ofl. und Bruch rotbraun, geglättet, $H$. noch $8,2 \mathrm{~cm}, \mathrm{Dm}$. $15-15,5 \mathrm{~cm}$ (Inv. Nr. 1132). Abb. 13,6.

1 a. Schale, fragmentiert, Ofl. und Bruch ziegelfarben, geglättet, Bodendm. 7,9-9,7 cm (o. Nr.). Abb. 13,10.

1 b. Henkel und Ausguß eines Kruges mit ausgeschnittenem Nacken, Ofl. schwarz, Bruch braun, Halsdm. ca. $5 \mathrm{~cm}$ (o. Nr.). Abb. 13,7.

2. Skyphos, Ofl. weißlich gelb, Bruch rotbraun, Firnis dunkelbraun (unten rot), auf dem Boden innen Kreisfläche von $0,7 \mathrm{~cm}$ Dm. ausgespart, Henkelinnenseiten tongrundig, Drehscheibenarbeit, Dm. 13,1-13,3 cm, H. $9,2 \mathrm{~cm}$ (Inv. Nr. 1135). Abb. 13,4.

3. Perle, Glaspaste, eingelegte Kreise grün und rot; Perle, Glaspaste, eingelegtes Band ausgefallen (o. Nr.). Abb. 17,25-26.

Grab VII

2. Zweihenkelige Schale, Henkel gebrochen, Ofl. und Bruch

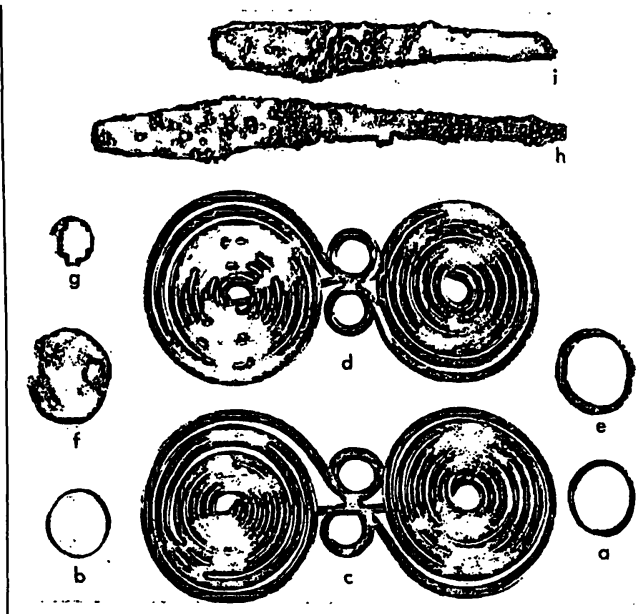

Abb.41. Vergina. Feld Lazaridis, Grabhügel. a-b Grab I; c-i Grab $V$

rotbraun, feine Kalkmagerung, geglättet, $D \mathrm{~m} .11 \mathrm{~cm}, \mathrm{H}$. 7,4 cm (Inv. Nr. 1143). Abb. 16,1.

3. Krug mit ausgeschnittenem Nacken, Ofl. braun, schwarze und rote Brennflecken, Bruch rotbraun mit grauschwarzem Kern, Ofl. verstrichen (senkrechte Glättspuren), H. $19 \mathrm{~cm}$ (Inv. Nr. 1141). Abb. 16,3.

4. Schale mit zwei randständigen Horizontalhenkeln, Ofl. rotbraun, Bruch graubraun, geglättet, $\mathrm{Dm}$. innen $14,5 \mathrm{~cm}, \mathrm{H}$. $6,5 \mathrm{~cm}$ (Inv. Nr. 1142). Abb. 16,4.

1. Scherben einer Knopfhenkelschale, Ofl. außen grau bis rotbraun, innen grau, Bruch grau bis rotbraun, geglättet, Dm. ca. 22 cm (Inv. Nr. 1143 a). Abb. 16,2.

1. Armspirale, Bronze, Dm. 4 cm (Inv. Nr. 850). Abb. 18,4.

3. Spirale einer Brillenfibel, Bronze, Dm. $1,9 \mathrm{~cm}$ (Inv. Nr. 851). Abb. 18,5.

Grab VIII

1. Spiralröllchen (?) (nicht nachweisbar).

3. Brillenfibel (nicht nachweisbar).

vielleicht zugehörig:

i. Krug mit ausgeschnittenem Nacken, Ofl. und Bruch rotbraun, geglättet, H. $13 \mathrm{~cm}$ (Inv. Nr. 1119). Abb.12,4.

Einzelfunde aus dem Bereich um Grab $I$

9-10. Zwei Gefäße (nicht nachweisbar).

1. Krug (Inv. Nr. 1119) siehe Grab VIII (Abb. 12,4).
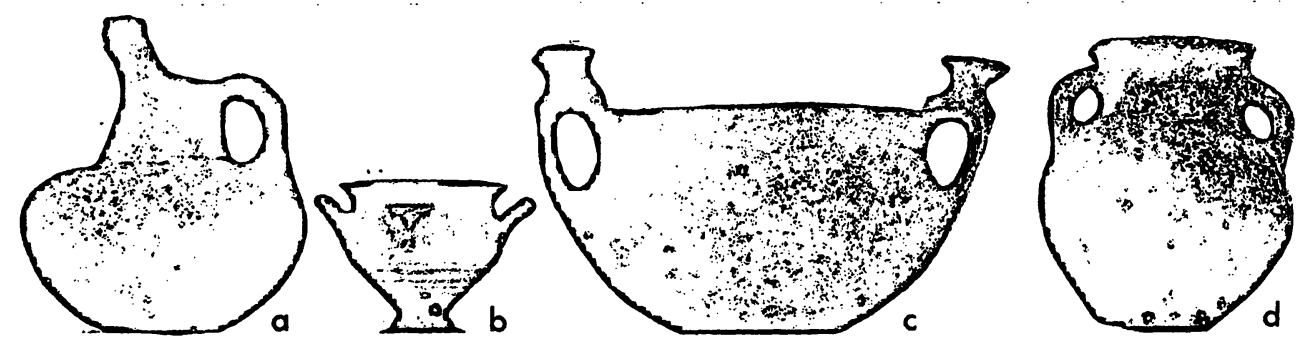

Abb. 42. Vergina. Feld Lazaridis, Grabhügel. a-c Grab VI; d Grab V 

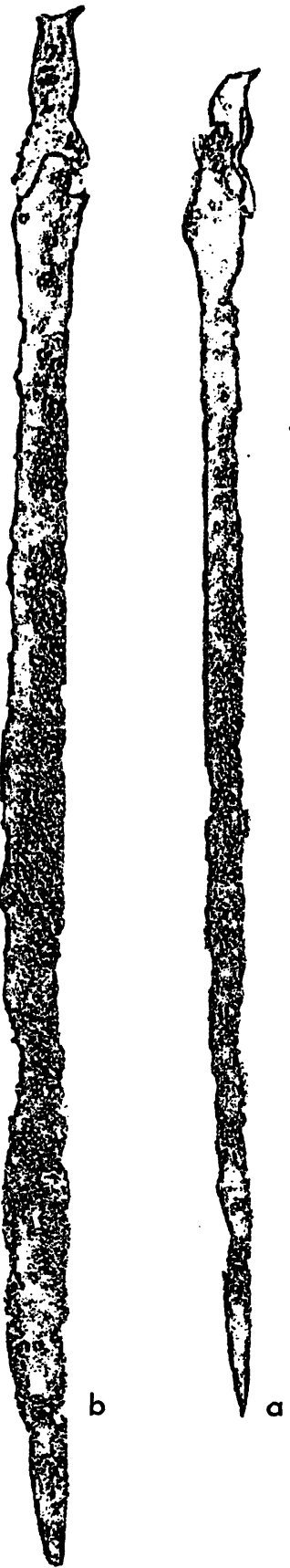

Abb.43. Vergina. Feld Lazaridis, Grabhügel. a Grab V/: b Grab I
11. Fragment ciner Armspirale, Bronze, Dm. $4,3 \mathrm{~cm}$ (Inv. Nr. 838). Abb. 7,25.

12. Drei Fragmente von Spiralröllehen, Bron\%e, L. noch $4,5 \mathrm{~cm}$ und $4,5 \mathrm{~cm}$ und $4 \mathrm{~cm}$ (Inv. Nr. 835). Abb. 7,26-28.

Aus der Hügelaufschüttung

a. Randfragment und Henkel cines Skyphos, Ofl. und Bruch gelblich wciß, Firnis rot, Drehscheibenarbeit, Dm. ca. $17 \mathrm{~cm}$ (o. Nr.). Abb. $16,9$.

b. Randfragment eines Skyphos, Ofl. und Bruch gelblich wciß, Firnis dunkelbraun, glän\%cnd, Drehscheibenarbeit, Dm. ca. $11 \mathrm{~cm}$ (o. Nr.). Abb. 16,6.

c. Fragment einer Knopthenkelschale (?), Ofl. und Bruch rotbraun, geglättet, $\mathrm{Dm} .16 \mathrm{~cm}$ (o. Nr.). Abb. 16,10.

Aus dem Hügel ohne nähere Angaben

- Fragment eines Tutulus mit Öse, Dm. $3 \mathrm{~cm}$ (o. Nr.). Abb. 18,20.

Feld Malamas, Grabhügel $\Gamma$

Grab I

21. Krug mit ausgeschnittenem Nacken, Ofl. hellgrau mit dunkelgrauen Brennflecken, Drehscheibenarbeit, H. $20,6 \mathrm{~cm}$ (Inv. Nr. 1147). Abb. 23,1.

22. Knopfhenkelschale, Ofl. lederfarben, geglättet, $\mathrm{Dm} .17 \mathrm{~cm}$, H. $11 \mathrm{~cm}$ (Inv. Nr. 1148). Abb. 23,3.

23. Krug mit ausgeschnittenem Nacken, Ofl. und Bruch rotbraun, fein gemagert, Ofl. verstrichen, H. $25,5 \mathrm{~cm}$ (Inv. Nr. 1150). Abb. 23,2.

24. Schale mit zwei randständigen Horizontalhenkeln, Ofl. rotbraun, verstrichen, Dm. innen $16,5 \mathrm{~cm}, \mathrm{H}$. $9 \mathrm{~cm}$ (Inv. Nr. 1149). Abb. 23,4.

1. Schwert, Eisen, Griffzunge gebrochen, L. noch $81,5 \mathrm{~cm}$ (Inv. Nr. 853). Abb. 25,1;

sechs Beschläge von der Schwertscheide (?), Eisen mit Bronzenieten: zwei schildförmige, L. $4,7 \mathrm{~cm}$ und $4,6 \mathrm{~cm}$; ein quadratischer, L. $4,5 \mathrm{~cm}$; ein rechteckiger, L. $5,2 \mathrm{~cm}$; zwei $\mathrm{H}$-förmige, L. $4,1 \mathrm{~cm}$ und $4 \mathrm{~cm}$ (Inv. Nr. 857). Abb. 25,8-13.

2. Lanzenspitze, Eisen, Tülle gebrochen, L. noch $29 \mathrm{~cm}$ (Inv. Nr. 856). Abb. 25, 15.

3. Sechs Pfeilspitzen, Eisen, L. $5,8 \mathrm{~cm}$ und $4,8 \mathrm{~cm}$ und $4,1 \mathrm{~cm}$ und $3,9 \mathrm{~cm}$ und $3,5 \mathrm{~cm}$ und $3,3 \mathrm{~cm}$ (Inv. Nr. 879). Abb. 25,2-7.

4. Kleines Messer mit gebogencm Rücken, Eisen, Reste vom Holzgriff, L. $8 \mathrm{~cm}$; unbestimmbare Eisenfragmente, Nadel (?) (Inv. Nr. 854). Abb. 25, 14.

5. Zwei Fingerringe mit gegenständigen Spiralenden, Bronze, Dm. $2 \mathrm{~cm}$ (Inv. Nr. 870-871). Abb. 25, 16-17.

6. Drillingsdoppelbeil, Bronze, mit Resten eines Eisenstiels, L. noch 26,3 cm (Inv. Nr. 855). Abb. 26,7.

7. 13 Fragmente von Spiralröllchen, Bronze, L. noch $15,7 \mathrm{~cm}$ und $11,8 \mathrm{~cm}$ und $11 \mathrm{~cm}$ und $11 \mathrm{~cm}$ und $9,7 \mathrm{~cm}$ und $9,5 \mathrm{~cm}$ und $7,5 \mathrm{~cm}$ und $5 \mathrm{~cm}$ und $4,5 \mathrm{~cm}$ und $3,6 \mathrm{~cm}$ und $3,2 \mathrm{~cm}$ und $2,1 \mathrm{~cm}$ (Inv. Nr. 869). Abb. 29,12-18.

8. Zwei Tutuli mit Öse, Bronze, auf einem Gewebereste, Dm. $3,7 \mathrm{~cm}$ (Inv. Nr. 865). Abb. 26,19-20.

9. Halsring, Bronze, Ofl. stark korrodiert, falsche Torsion, drei aufgeschobene Drahtringchen, Dm. $12 \mathrm{~cm}$ (Inv. Nr. 874);

Halsring, Bronze, falsche Torsion, Dm. $12,7 \mathrm{~cm}$ (Inv. Nr. 875).

Abb. 29,3-4; 45,d-e.

10. Ringanhänger, Bronze, Ōse gebrochen, anhaftende Gervebereste, Dm. 6,3 cm (Inv. Nr. 864). Abb. 26,5; 45,f.

11. Kleiner Ring, Bronze, beidseits Gewebereste, Dm. ca. 2,5 cm (Inv. Nr. 877). Abb. 29,9. 

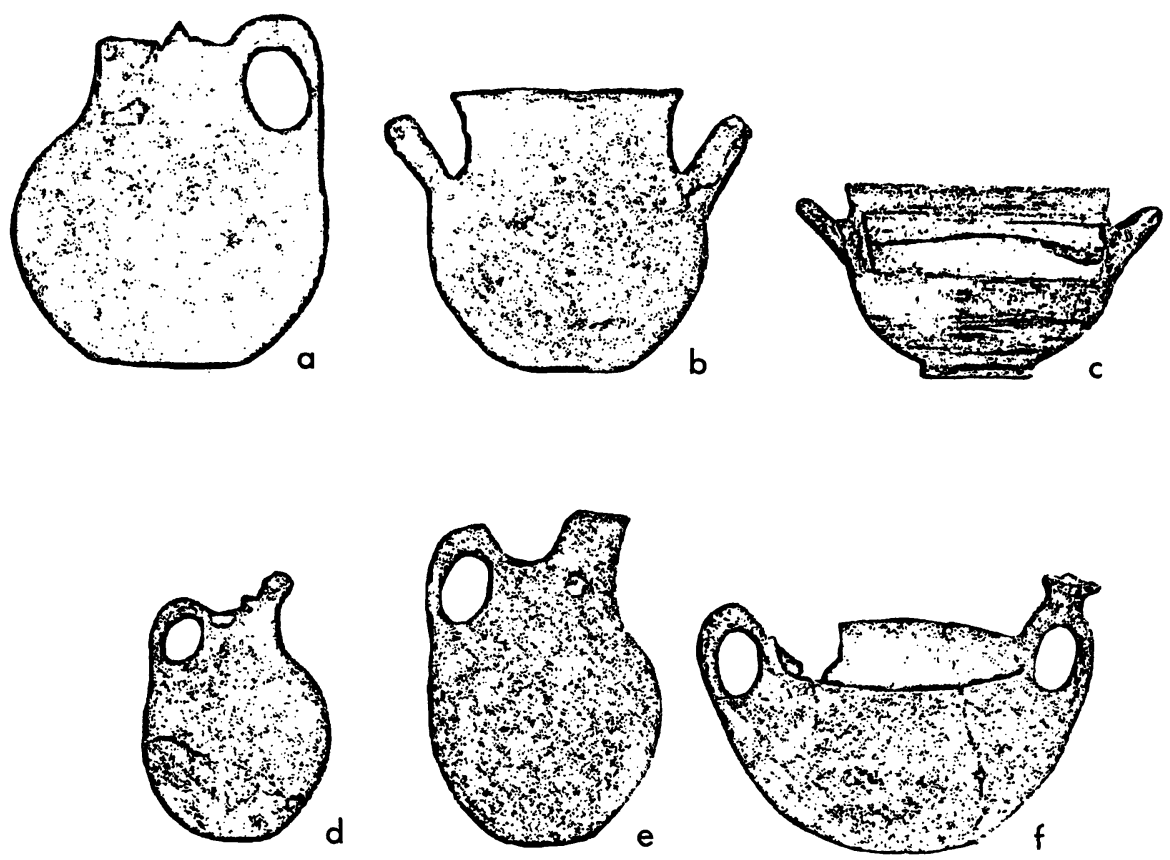

Abb. 44. Vergina. Feld Malamas, Grabhügel B. a-f Grab $I I$ (a-b. e Grabzugehörigkeit fraglich)

12. Zwei Lockenringe, Gold, Dm. $1,9 \mathrm{~cm}$ (Inv. Nr. 878). Abb. 29,10-11.

13. Zwei große Brillenfibeln, Bronze, L. $19 \mathrm{~cm}$ und $19,3 \mathrm{~cm}$ (Inv. Nr. 868). Abb.29,1-2.

14. Armspirale, Bronze, Enden mit Strichdekor, Dm. unten $7,7 \mathrm{~cm}$ (Inv. Nr. 866). Abb. 25,18.

15. Drei kleine Brillenfibeln, Bronze, eine mit Geweberesten, L. $4,1 \mathrm{~cm}$ und $3 \mathrm{~cm}$ und $3,7 \mathrm{~cm}$ (Inv. Nr. 872-873.876). Abb. 29,5-7.

16. Falere, Bronze, auf der Krempe getriebener Buckeldekor, Gewebereste, Öse verlötet, mit Eisenrost überzogen, Dm. 11,2 cm (Inv. Nr. 858). Abb. 26,9;

Falere, Bronze, auf der Krempe getriebener Buckeldekor, auf der Unterseite Gervebereste, Öse von Eisenrost überzogen, Dm. 10,6 cm (Inv. Nr. 859). Abb. 26,8;

Falere, fragmentiert, antik geflickt, auf der Krempe getriebener Buckeldekor, Öse verlötet, Oberseite verkrustet mit Gewebe- oder Fellabdrück, Dm. $11 \mathrm{~cm}$ (Inv. Nr. 861.877). Abb. 26,6;

Falere, fragmentiert, Bronze, am Rand der Krempe Buckelreihe, Öse mitgegossen, an der Unterseite Gervebereste, Dm. 7,5 cm (Inv. Nr. 863). Abb. 26,2;

Falere, Bronze, Öse verlötet, Gewebereste, Dm. 7,2 cm (Inv. Nr. 860). Abb. 26,1;

flacher Tutulus mit Öse, am Rand Buckelreihe, verkrustet, Gewebereste, Dm. 7,6 cm (Inv. Nr. 862). Abb. 26,4.

17. Ca. 57 kleine Tutuli mit Dorn, Bronze, Dm. 0,9 cm (Inv. Nr. 879 a). Abb. 26,10;

ca. 126 Tutuli mit Öse, Bronze, Dm. $2 \mathrm{~cm}$ und $1,6 \mathrm{~cm}$ und $1 \mathrm{~cm}$ (Inv. Nr. 879 a). Abb. 26,11-18.

18. Radanhänger, Bronze, anhaftende Reste von grobem Gewebe, Dm. 4,5 cm (Inv. Nr. 867). Abb. 26,3; 45,c.

19. Blechscheibe mit Mittelbuckel, Bronze, Gewebereste, Dm. $3 \mathrm{~cm}$ (Inv. Nr. 877). Abb. 29,8.
20. 54 Perlen, kugelig bis scheibenförmig, Sard (?) (Abb. 29,21-27); eine kugelige Perle, Bergkristall (Abb. 29,20); eine Scheibenperle, Fayence (?) (Inv. Nr. 1180). Abb. 29,19; $45, a-b$.

- 17 menschliche Zähne von zwei Individuen (siehe Anhang 2).

\section{Grab II}

1. Krug mit ausgeschnittenem Nacken, Ofl. und Bruch grau, geglättet, H. 19,5 cm (Inv. Nr. 1151). Abb. 23,5; im Krug Spiralscheibe einer Brillenfibel, Bronze, Dm. noch $1,5 \mathrm{~cm}$ (o. Nr.). Abb. 30,11.

2. Zwei vollständige Spiralröllchen, Bronze, L. $15,2 \mathrm{~cm}$ und $13,2 \mathrm{~cm}$;

sieben Fragmente von Spiralröllchen, Bronze, L. noch $13,5 \mathrm{~cm}$ und $14,1 \mathrm{~cm}$ und $12,1 \mathrm{~cm}$ und $9,7 \mathrm{~cm}$ und $11,1 \mathrm{~cm}$ und $9,7 \mathrm{~cm}$ und $3,7 \mathrm{~cm}$ (Inv. Nr. 880).

Abb. 30,1-9.

3. Drei Tutuli mit Öse, Bronze, Dm. $3,5 \mathrm{~cm}$ und $3 \mathrm{~cm}$ und $3 \mathrm{~cm}$ (Inv. Nr. 881). Abb. 30,12-14.

4. Zwei kleine Ringe, Bronze, offen, Dm. 2,1 cm (Inv. Nr. 882). Abb. 30,15-16.

5. Kleiner Ring, Bronze, gegossen, Dm. 3,1 cm (Inv. Nr. 882). Abb. 30,17.

6. Fragment eines Halsrings, Bronze, tordiert, zu kleinem Ring gebogen, Dm. 3,2 cm (Inv. Nr. 883). Abb. 30,10.

- 19 menschliche Zähne von zwei Individuen (siehe Anhang 2).

\section{Grab III}

1. Krug mit ausgeschnittenem Nacken, Ofl. rotgrau bis grau, fein gemagert, geglättet, H. $25,4 \mathrm{~cm}$ (Inv. Nr.1152). Abb. 24,3.

2. Krateriskos, Ofl. braungrau, Bruch grau, fein gemagert, 

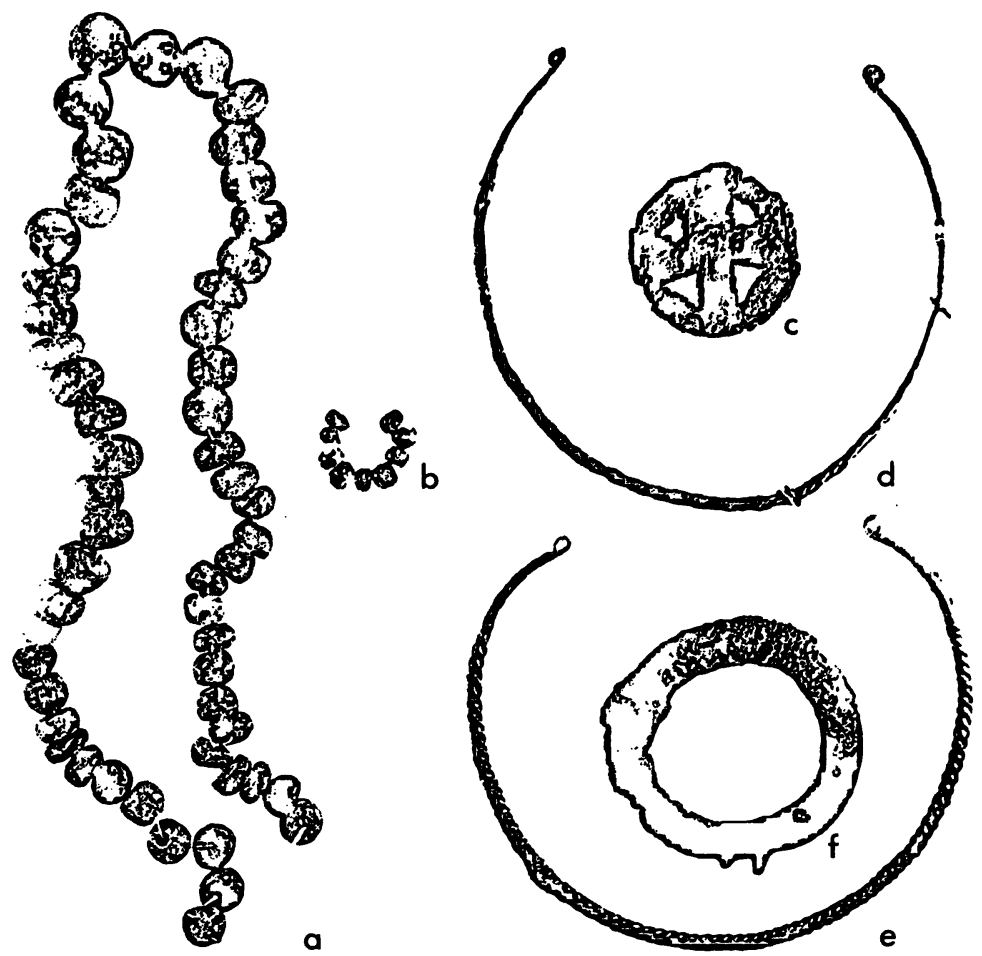

Abb. 45. Vergina. Feld Malamas, Grabhügel $\Gamma$. a-f Grab I

geglättet, hart gebrannt, Dm. $19,1-19,5 \mathrm{~cm}$, H. $21,5 \mathrm{~cm}$ (Inv. Nr. 1153). Abb. 24,1.

3. Schwert, Eisen, Spitze gebrochen, auf Schulter und Griffzunge geringe Reste vom Griffbelag aus Holz (?), L. noch 110,5 cm (Inv. Nr. 884). Abb. 30,18.

4. Fragment eines kleinen Messers, Eisen (Inv. Nr. 886).

5. Messer, Eisen, Reste vom Griff aus Holz (?), L. $18,6 \mathrm{~cm}$ (Inv. Nr. 885). Abb. $30,19$.

6. Zwei Fragmente einer Nadel (?), Eisen (Inv. Nr. 887).

Grab IV

1. Saugkännchen, Ofl. beige, Bruch graubraun, geglättet, $H$. $10,3 \mathrm{~cm}$ (Inv. Nr. 1155). Abb. 24,5; 46,b.

2. Skyphos, Ofl. gelblich weiß, Firnis schwarz, matt, Dm. $18,6 \mathrm{~cm}$, H. $13 \mathrm{~cm}$ (Inv. Nr. 1156). Abb. 24,2; 46,a.

2a. Fragmente vom Ausguß eines Kruges mit ausgeschnittenem Nacken, Ofl. und Bruch braungrau (o. Nr.). Abb. 24,4.

Grab V

1. Knopfhenkelschale, Ofl. graubraun bis rotbraun, verstrichen, Dm. 15,5-16,5 cm, H. $13 \mathrm{~cm}$ (Inv. Nr. 1154). Abb. 24,6.

2. Brillenfibel, Bronze, L. $3,7 \mathrm{~cm}$ (Inv. Nr. 888). Abb. 18,6.

Grab VI

1. Unterteil eines Kruges (?), Ofl. und Bruch rotbraun, geglättet, H. noch 13,2 cm (Inv. Nr. 1158). Abb. 31,3.

2. Skyphos, Rand gebrochen, Ofl. und Bruch rötlich gelb, Firnis rot, innen kräftige Drehrillen, Henkelinnenseiten tongrundig, H. noch 10,7 cm (Inv. Nr. 1157). Abb.31,1.
Grab VII

1. Knopfhenkelschale, Ofl. lederfarben, geglättet, $\mathrm{Dm}$. $14-15,2 \mathrm{~cm}, \mathrm{H} .8,7 \mathrm{~cm}$ (Inv. Nr. 1159). Abb. 31,4.

2. Schale mit zwei randständigen Horizontalhenkeln, Ofl. lederfarben (teilweise abgeplatzt), Dm. außen $20,3 \times 19,4 \mathrm{~cm}$ (Inv. Nr. 1160). Abb. 31,5.

3. Krug mit ausgeschnittenem Nacken, Ofl. grau bis graubraun, Bruch grau, fein gemagert, geglättet, H. $25 \mathrm{~cm}$ (Inv. Nr.1161). Abb.31,2.

\section{Grab VIII}

1. Skyphos, Ofl. weißlich, Firnis rotbraun, innen breite Drehrillen, Standring innen tongrundig, auf Bodenunterseite gemalte Kreisfläche, Drehscheibenarbeit, Dm. 13,7-14 cm, H. $10 \mathrm{~cm}$ (Inv. Nr. 1163). Abb. 31,7; 47,b.

2. Topf, Henkel gebrochen, Ofl. und Bruch rotbraun, Unterteil aufgerauht, Dm. 20,6-20,9 cm, H. 22,5 cm (Inv. Nr. 1162). Abb. 31,6; 47,a.

3. Armring, Bronze, Dm. 4 cm (Inv. Nr. 889). Abb. $29,29$.

\section{Grab IX}

1. Saugkännchen, Ofl. gelblich weiß, Bruch graugelb, Firnis braun bis schwarz, dünn, streifig, Drehscheibenarbeit, H. $13 \mathrm{~cm}$ (Inv. Nr.1164). Abb. 32,1; 48,c.

2. Krug mit ausgeschnittenem Nacken, Ofl. lederfarben, Bruch grau, schwach gebrannt, H. 18,8 cm (Inv. Nr. 1168). Abb. 32,3; 48,a.

3. Knopfhenkelschale, Ofl. und Bruch hell lederfarben bis rotbraun, geglättet, Dm. $15 \mathrm{~cm}, \mathrm{H} .8,5 \mathrm{~cm}$ (Inv. Nr. 1166). Abb. 32,5. 

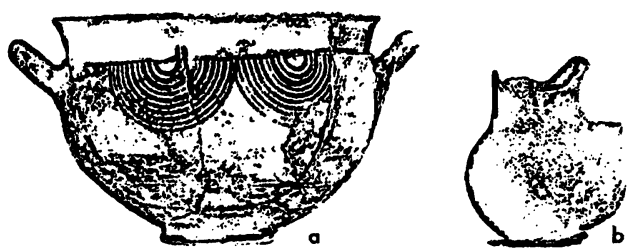

Abb. 46. Vergina. Feld Malamas, Grabhügel $\Gamma$. a-b Grab IV

4. Einhenkeliger Becher, Ofl. lederfarben, verstrichen, Dm. 14-14,5 cm (Inv. Nr.1167). Abb. 32,4; 48,d.

5. Skyphos, Ofl. gelblich weiß, Firnis schwarz, Standfläche tongrundig, auf dem Boden innen Kreis von $1,8 \mathrm{~cm} \mathrm{Dm}$. ausgespart, Henkelinnenseiten tongrundig, Dm. 14,4-14,8 cm, H. 9 cm (Inv. Nr. 1165). Abb. 32,2; 48,b.
6. Messer, Eisen, Spitze gebrochen, Griff aus Bein, L. noch $10 \mathrm{~cm}$ (Inv. Nr. 891);

Messer, Eisen, Klinge gebrochen, L. noch $6,5 \mathrm{~cm}$ (lnv. Nr. 891).

Abb. 18,2-3.

7. Tutulus mit Öse, Bronze, Dm. 1,6 cm (o. Nr.).

Grab $X$

1. Krug mit ausgeschnittenem Nacken, Ofl. und Bruch rotbraun, geglättet, H. $13 \mathrm{~cm}$ (Inv. Nr. 1169). Abb.32,6.

2. Hälfte einer Knopfhenkelschale, Ofl. braun, rotbraune und schwarze Brennflecken, Bruch braun, Dm. $20 \mathrm{~cm}, \mathrm{H}$. $10,5 \mathrm{~cm}$ (Inv. Nr. 1170). Abb. 32,7.

3. Nicht mehr identifizierbare Beigabe (Spiralröllchen?).

3 a. Fragment eines Spiralröllchens, Bronze, L. noch $3,2 \mathrm{~cm}$ (o. Nr.). Abb. 18,7.

4. Scheibenperle, Fayence (?), Dm. 0,9 cm (o. Nr.). Abb. 18,8.

Grab XI

1. Krug mit ausgeschnittenem Nacken, Ofl. grau bis rotbraun, Bruch beim Griff grau mit rotbraunem Kern, geglättet, $H$. 18,1 cm (Inv. Nr. 1171). Abb. 32,8.

2. Knopfhenkelschale (nicht nachweisbar).
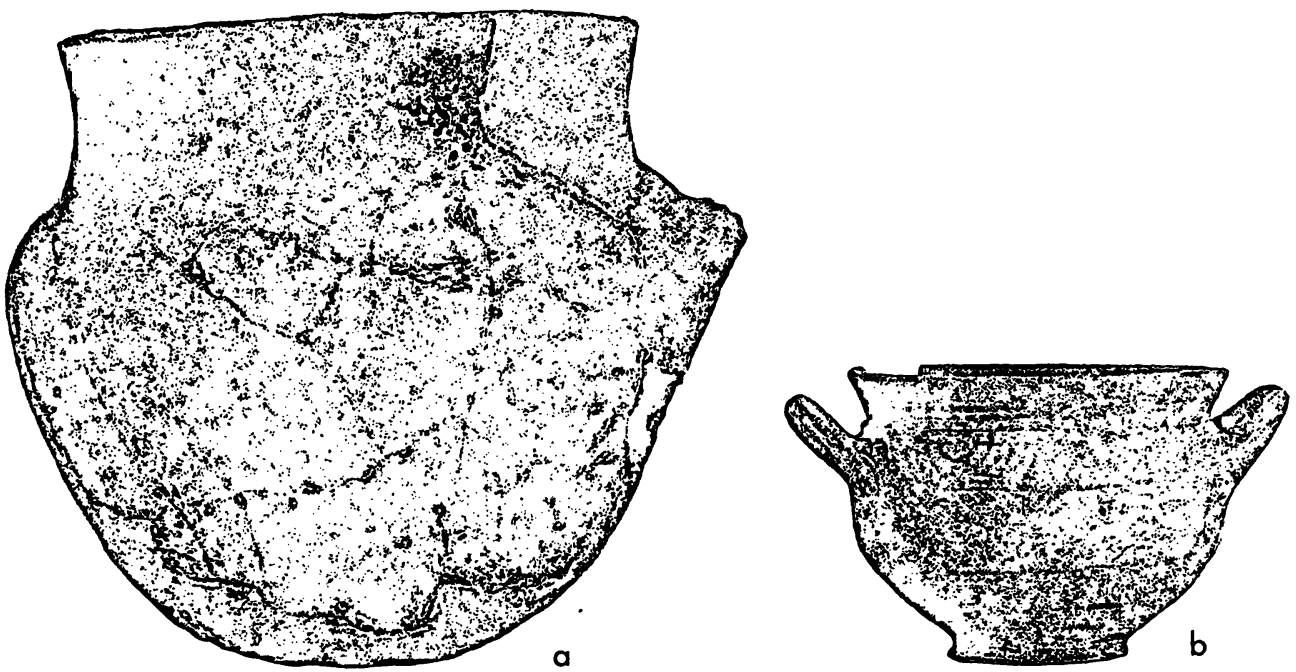

Abb. 47. Vergina. Feld Malamas, Grabhügel $\Gamma$. $a-b$ Grab VIII
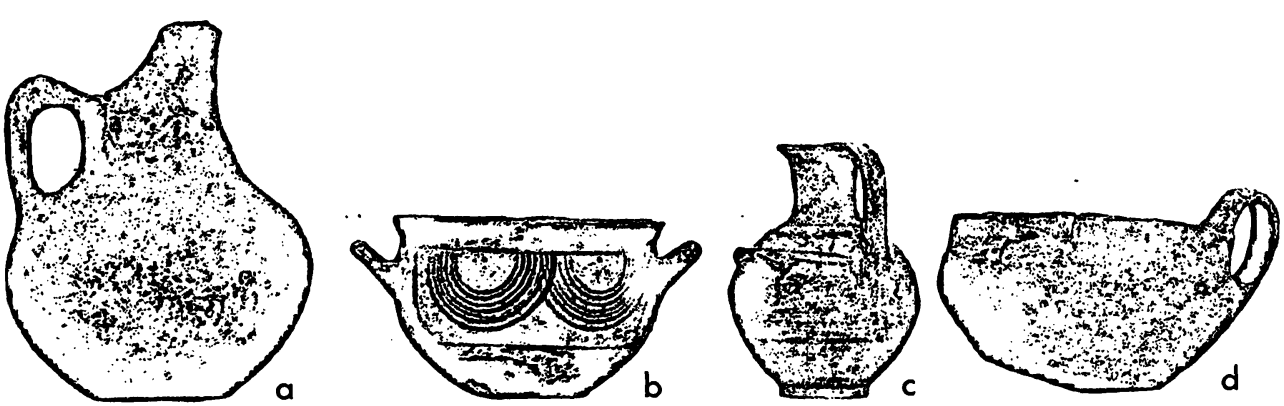

Abb. 48. Vergina. Feld Malamas, Grabhügel $\Gamma$. a-d Grab IX 
Grab XII

1. Amphoriskos, Ofl. wcißlich, Firnis schwarzbraun, abgericben. Henkelinnenscite tongrundig, Drehscheibenarbeit, H. $15 \mathrm{~cm}$ (Inv. Nr. 1172). Abb. 34,3.

2. Krug mit ausgeschnittenem Nacken, Ofl. hellbraun mit rotbraunen Brennflecken, geglättet, H. $22 \mathrm{~cm}$ (Inv. Nr. (173). Abb.34,1.

3. Krug mit ausgeschnittenem Nacken, Ofl. rotbraun, geglättet, H. $10 \mathrm{~cm}$ (Inv. Nr. 1174). Abb. 34,4.

4. Knopfhenkelschale, Ofl. hell lederfarben, geglättet, H. $8,5 \mathrm{~cm}$ (Inv. Nr.1175). Abb. 34,6.

5. Schale mit zwei randständigen Horizontalhenkeln, Ofl. und Bruch rotbraun, geglättet, Dm. außen $21,5 \mathrm{~cm}, \mathrm{H} .7,8 \mathrm{~cm}$ (Inv. Nr. 1177). Abb. 34,2.

6. Krug mit ausgeschnittenem Nacken, Ofl. rotbraun, Bruch rotbraun mit grauem Kern, geglättet, $H .15,3 \mathrm{~cm}$ (Inv. Nr. 1176). Abb. 34,5.

Einzelfund XIII

- Knopfhenkelschale, fragmentiert, Ofl. und Bruch lederfarben, fein gemagert, geglättet, schwach gebrannt, $D \mathrm{~m} .17 \mathrm{~cm}$, H. 10,5 cm (Inv. Nr. 1178). Abb. 34,7.

Einzelfund XIV

- Zwei Fragmente einer Armspirale, Bronze, Dm. 4,9cm (Inv. Nr. 893). Abb. 18,10.

Fundgruppe $X V$ (wohl zerstörtes Grab)

- Krug mit ausgeschnittenem Nacken, Ofl. und Bruch rotbraun, geglättet, H. noch $20 \mathrm{~cm}$ (Inv. Nr. 1179). Abb. 34,8; Halsring, Bronze, tordiert, Dm. 11,3 cm (Inv. Nr. 894). Abb. 29,28.

Pithos 1

1. Becher, einhenkelig, Ofl. lederfarben bis rotbraun, Bruch lederfarben, gut geglättet, $\mathrm{Dm} .16 \mathrm{~cm}$ (o. Nr.). Abb. 35,1.

2. Fragmente eines offenen Gefäßes (Becher oder Schale), Ofl. und Bruch ziegelfarben, geglättet, $\mathrm{Dm} .13-14 \mathrm{~cm}$ (o. Nr.). Abb. 35,3 .

3. Brillenfibel, Bronze, L. 8,6 cm (Inv. Nr. 895). Abb. 29,31.

Streufunde aus dem Bereich der Steinsetzungen

- Krug mit ausgeschnittenem Nacken, Ofl. grau, teilweise mit graubrauner Brennhaut, rauh, Bruch grau bis graubraun, dünnwandig, hart gebrannt, $H$. 16,7 cm (Inv. Nr.1144). Abb. 35,4

- Hälfte einer Knopfhenkelschale, Ofl. und Bruch lederfarben, geglättet, Dm. ca. $12 \mathrm{~cm}$ (Inv. Nr. 1145), dabei eiserne Lanzenspitze (im Museum nicht nachweisbar). Abb. 35,12.

- Schale mit zwei randständigen Horizontalhenkeln, Ofl. ziegelfarben, geglättet, $\mathrm{Dm} .19,5 \times 22 \mathrm{~cm}, \mathrm{H}$. $7,5 \mathrm{~cm}$ (Inv. Nr. 1146). Abb. 35,5.

- Außerdem Fragmente von mindestens vier weiteren Knopfhenkelschalen (Abb. 35,2.8-9.11); drei Schalen mit zwei Griffen und zwei Grifflappen (Abb. 35,6) sowie

einer Schale mit zwei randständigen Horizontalhenkeln (Abb. 35,7).

- Nadel mit Kegelkopf, Bronze, Spitze gebrochen, L. noch 23,5 cm (Inv. Nr. 897). Abb. 18,25.

- Messer, Eisen, stark korrodiert, L. noch $9 \mathrm{~cm}$ (o. Nr.). Abb. 18,19.

Streufunde im Bereich der einfachen Grabgruben

$\zeta .9 . \kappa-\lambda$. Vier kleine Ringe, gegossen, Dm. $2,8 \mathrm{~cm}$ und $2,7 \mathrm{~cm}$ und 2,6 cm und 2,3 cm (Inv. Nr. 900). Abb. 18,11-12.15.17.

$\eta$. Golddraht, zu einer Spirale mit vier Windungen gebogen (Inv. Nr. 902). Abb. 18,13.

t. Fragment eines Spiralröllchens, Bronze, L. noch $8,9 \mathrm{~cm}$ (o. Nr.). Abb. 18,22.

$\mu$. Fragment eines Armrings, Bronze, zu kleinem Ring gebogen, Dm. 2,3 cm (Inv. Nr. 900). Abb. 18,11. v. Brillenfibel, Bronze, L. 14,1 cm (Inv. Nr. 898). Abb. 18,14.

a. Lockenring, Gold, Dm. 2,1 cm (Inv. Nr. 899). Abb. 18,18.

$\beta$. Lanzen- oder Pfeilspitze, Eisen (nicht nachweisbar).

$\gamma$. Fragment eines Spiralröllchens, Bronze, L. noch $4,1 \mathrm{~cm}$ (o. Nr.). Abb. 18,23.

8. Nadel mit Kegelkopf, Bronze, Schaft gebrochen, L. noch $12,4 \mathrm{~cm}$ (Inv. Nr. 896). Abb. 18,24.

ع. Tutulus, Bronze, Ose gebrochen, Dm. $3,2 \mathrm{~cm}$ (o. Nr.). Abb. 18,21.

zu VIII (?): Wetzstein, grauer Stein, L. $8,8 \mathrm{~cm}$ (Inv. Nr. 890). Abb. 29,30.

zu $X I I$ (?): Kleiner Ring, Bronze, gegossen, Dm. 2,7 cm (Inv. Nr. 892). Abb. 18,9.

Weitere Streufunde von Hügel $\Gamma$

aus der Planierung:

- Tülle einer Lanzenspitze, Eisen, L. noch $19,3 \mathrm{~cm}$ (o. Nr.). Abb. $18,27$.

von der Oberfläche:

- Dreikantige Tüllenpfeilspitze, Eisen, L. $9 \mathrm{~cm}$ (o. Nr.). Abb. 18,26.

aus der Hügelaufschüttung:

- Henkelfragment eines Kruges (?) mit pastoser Bemalung (o. Nr.). Abb. 35,10;

- drei Scherben eines Skyphos, dünnwandig, Firnisbemalung, Drehscheibenarbeit (o. Nr.). Abb. 35,13.

Aus einem der 1970 untersuchten Hügel

- Kleines Spiralröllchen, komplett, L. $3,9 \mathrm{~cm}$ (o. Nr.). Abb. 18,34;

- zwei anpassende Fragmente von Spiralröllchen, L. noch $4,9 \mathrm{~cm}$ (o. Nr.). Abb. 18,33;

- zehn Fragmente von Spiralröllchen, L. noch $4-2,2 \mathrm{~cm}$ (o. Nr.). Abb. 18,28-32.35-39.

\section{Literaturverzeichnis}

Andronikos 1969: M. Andronikos, Vergina I / To nekrotapheion ton tymbon (1969).

Heurtley u. Skeat 1930-31: W. A. Heurtley u. T. C. Skeat, The Tholos Tombs of Marmariane. Annu. Brit. School Athens 31, 1930-31, $2 \mathrm{ff}$.

Kilian 1975 a: K. Kilian, Trachtzubehör der Eisenzeit zwischen Ägäis und Adria. Prähist. Zeitschr. 50, 1975, 9 ff.

- 1975 b: -, Fibeln in Thessalien von der mykenischen bis zur archaischen Zeit. PBF XIV 2 (1975).

Kilian-Dirlmeier 1979: I. Kilian-Dirlmeier, Anhänger in Griechenland von der mykenischen bis zur spätgeometrischen Zeit. PBF XI 2 (1979).

Kraiker u. Kübler 1939: W. Kraiker u. K. Kübler, Kerameikos I (1939).

Kübler 1943: K. Kübler, Kerameikos IV (1943).

- 1954: -, Kerameikos V 1 (1954).

Lefkandi I: M.R. Popham u.a., Lefkandi I / The Iron Age (1980).

Müller-Karpe 1962: H: Müller-Karpe, Die Metallbeigaben der früheisenzeitlichen Kerameikos-Gräber. Jahrb. DAI 77, 1962, $59 \mathrm{ff}$.

Petsas 1961-62: Ph. Petsas, Anaskaphai archaiou nekrotapheiou Verginas. Arch. Deltion 17, 1961-62 Meletai $218 \mathrm{ff}$.

- 1963: -, Vergina. Arch. Deltion 18, 1963 Chronika 217 ff.

Vokotopoulou 1986: I. Vokotopoulou, Vitsa / Ta nekrotapheia mias molossikis komis (1986). 


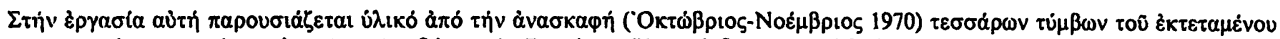

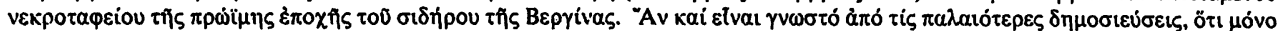

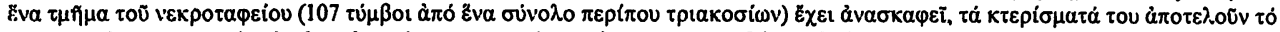

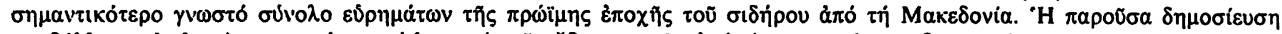

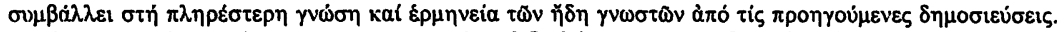

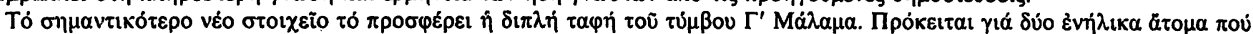

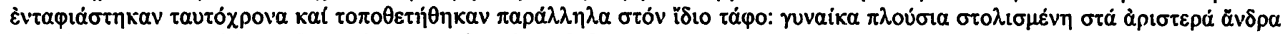

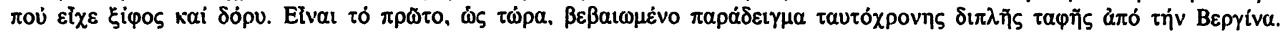

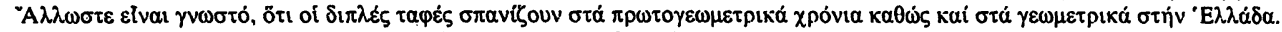

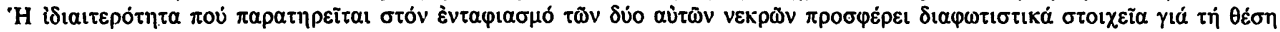

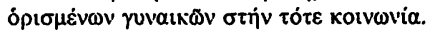

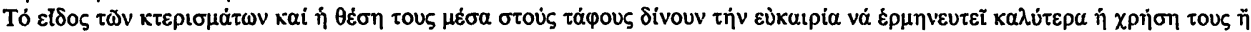

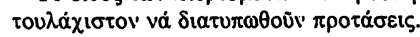

Katerina Rhomiopoulou, Nationalmuseum Athen, Tositsa 1, GR 10682 Athen

Imma Kilian-Dirlmeier, Karthäuserstr. 32, D 5300 Bonn 
Appendix 1

\title{
Metallographic Investigation of Iron Artefacts from EIA Cemetery at Vergina
}

\author{
by Effi Photos, Athens
}

Seven iron artefacts from Vergina (Lazarides and Malama tombs) excavated in 1970 by K Romiopoulou and presently at Veria Muscum were sampled for analysis in January 1988. The objects included three swords, two knives, one spit and one arrowhead in a varying degree of prescrvation ranging from being substantially corroded (severe flaking) to containing a consolidated corrosion layer. No conscrvation treatment was undertaken prior to their storage in 1970 .

A section was cut across the objects at or near the tip and, in one occasion, at the hilt. The sections were analysed a) metallographically, to establish the degree of stecling (extent of carbon content in the iron and the type of heat treatment the object had undergone) and b) chemically, by Electron Probe Microanalysis (EPMA) to determine the chemical composition of impurities in the metal (other than carbon which can be determined metallographically) and that of slag inclusions trapped in the metal. Chemical analysis is aimed at attempting to establish the type of iron ore and eventually its provenance.

\section{Methodology}

The sections were embedded in a cold setting resin, ground with a series of abrasive papers and subsequently polished with three different diamond pastes $(6 ; 1 ; 1 / 4$ microns). After polishing the samples were etched with $2 \%$ nital and examined optically with the metallographic microscope. The same polished sections were examined chemically in the EPMA, a Cambridge Scientific Instruments Mark V attached to a Link 860 X-ray microanalyser, providing spot elemental analysis.

\section{Analytical Results}

\section{Metallographic Observations}

a. Swords

823. 9th c BC. Length: $75 \mathrm{~cm}$ without hilt $(10 \mathrm{~cm})$. Width (max): $5 \mathrm{~cm}$. Extensively corroded and broken at two points: a) near the base of the hilt and b) $12 \mathrm{~cm}$ from the tip. Metallographic section was cut at $27 \mathrm{~cm}$ from the tip.

This section can be divided in two subsections consisting of the cutting edge and the dull edge. The dull edge is primarily ferritic, containing Neumann bands, the result of stress induced on the grains following cold working at temperatures below $500^{\circ} \mathrm{C}$. The section comprising the cutting edge consists of ferrite with pearlite at the grain boundaries $\left(.15-.20 \% \mathrm{C}\right.$ ). Hardness: $\mathrm{Hv}_{(100)}=132$ (fer- rite), $\mathrm{Hv}_{(100)}=158$ (ferrite and pearlite). It is possible that two shects of metal, one of low carbon and the other of mild steel were forge-welded together in an edge to edge layering. However, there is no clear indication of slag stringers corroborating this fact. In addition, the "weldline" over the entire length of the section is hardly straight. It is likely that this object was either produced from one piece of the bloom with uneven carbon content or that the same object got decarburised along one edge while at the forge.

833. 9th-8th c BC. Length: $39.5 \mathrm{~cm}$. Width (max): $3 \mathrm{~cm}$. Very good state of preservation. Metallographic section cut $9 \mathrm{~cm}$ from the tip.

This is the best preserved artefact. There is evidence for gradual decrease in carburisation from the cutting edge to the dull edge (from $.40 \%$ to $.15 \% \mathrm{C}$ ) displaying ferrite of widmanstatten structure (Fig. 49). This effect is the result of fast cooling in air. The cutting edge is ferrite and pearlite $(.4 \% \mathrm{C})$, the pearlite being partly spheroidised. Spheroidisation implies prolonged heating between $650-700^{\circ} \mathrm{C}$. The object must have been removed from the forge after having been exposed to the reducing environment of the hearth for some time to allow gradual diffusion of carbon into the blade. $\mathrm{Hv}_{(100)}=174-242$. Smooth gradient in carburisation implies good control of the reducing environment in the forge and suggests a high technical skill from the part of the smith.

853. Late 9th c - early 8th c BC. Length: $80.5 \mathrm{~cm}$ with hilt. Width (max): $4 \mathrm{~cm}$. Two metallographic sections were cut, one $3.5 \mathrm{~cm}$ from the tip and another at the edge of the hilt.

Uniform carbon content and grain distribution of ferrite and pearlite throughout the section $(.20 \% \mathrm{C})$ with the exception of one edge (probably the cutting edge) characterised by elongated grains (Fig. 50) the result of cold working, a function which can impart hardness on the steel. Hardness: $\mathrm{Hv}_{(100)}=196$ (pearlite and ferrite). There exist some long slag inclusions but they are generally few.

853 hilt. The section consists of large equiaxial ferrite grains exhibiting age hardening, namely fine carbide which has come out of the ferrite as a result of prolonged burial. $\mathrm{Hv}_{(100)}=196$ (ferrite and pearlite). There exist small scattered slag inclusions, some of them elongated and consisting of two phases, an iron oxide and a glassy silicate phase.

It is clear that the hilt and blade are metallographically different; the hilt containing very little carbon and the blade containing not only a substantial amount thereof but also distributed in a gradient across the section. 


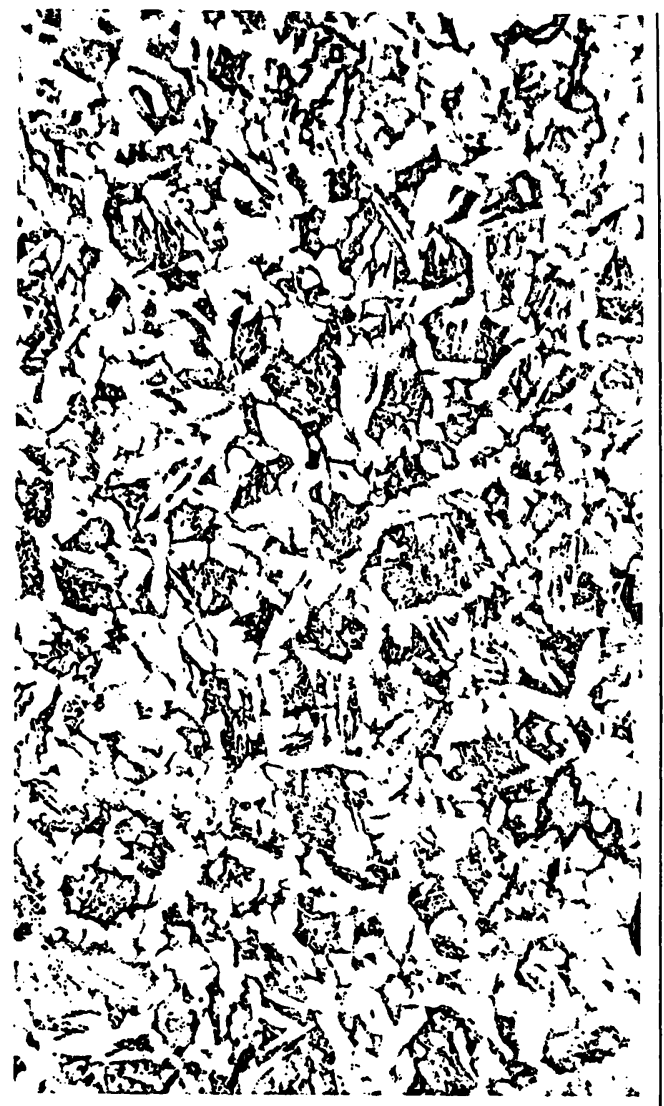

Fig. 49. Sword Inv. Nr.833. Ferrite grains of widmanstatten structure with pearlite. Scale 400:1

\section{b. Knives}

988. Surface find ${ }^{236}$. Length: $18.5 \mathrm{~cm}$. Width: $2 \mathrm{~cm}$. Metallographic section cut $9.5 \mathrm{~cm}$ from the tip.

The section consists of ferrite with pearlite at the grain boundaries, pearlite being spheroidised. The degree of carburisation ranges between $.10-.25 \% \mathrm{C}$, while the grain distribution is quite uniform. Spheroidised pearlite is formed when steel is heated for a prolonged period below $700^{\circ} \mathrm{C}$. Heating took place in a well controlled reducing environment ensuring uniform carbon diffusion throughout the sample and equally uniform grain distribution. There exists a large number of slag inclusions, some elongated as slag stringers (Fig. 51). It is more likely that the blade was made from one single sheet of metal rather than two.

854. 9th-8th c BC. Length: $6.4 \mathrm{~cm}$. Width (max): $1.5 \mathrm{~cm}$. Metallographic section cut $9.5 \mathrm{~cm}$ from the tip.

236 "Surface find from the same area of the Early Iron Age cemetery but not found during the excavation the report of which is presented here.

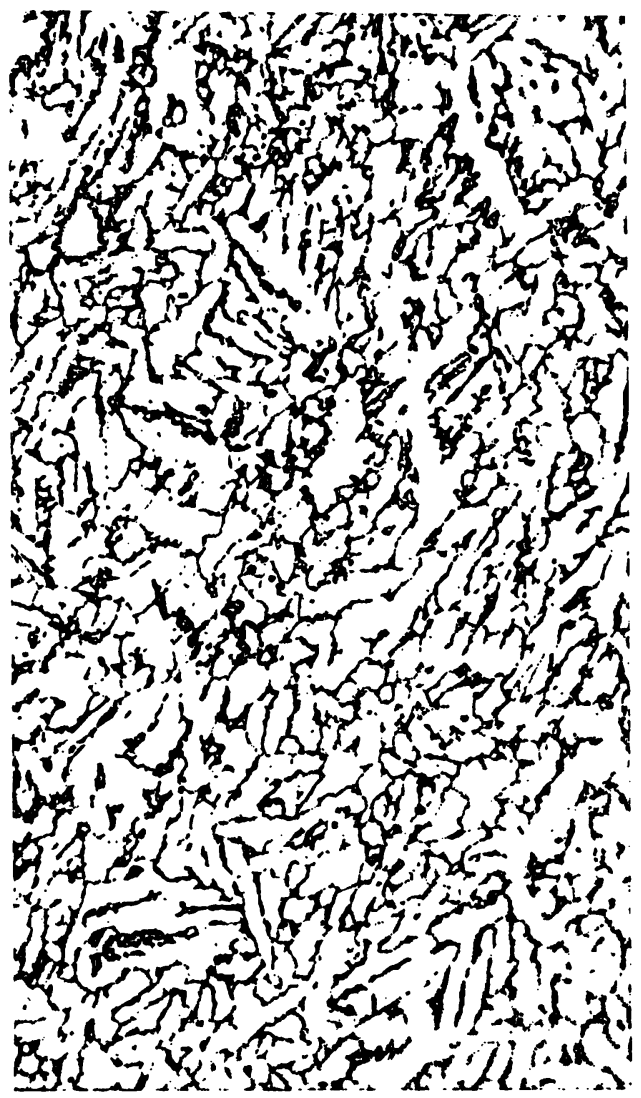

Fig. 50. Sword Inv. Nr. 853. Elongated ferritic grains with pearlite at the grain boundaries. Scale 200:1

The cutting edge consists of martensite produced from fast cooling by quenching into water. Moving towards the dull edge the structure changes gradually to ferrite and pearlite of the widmanstatten structure to a lower carbon area $(.15-.20 \% \mathrm{C})$. Martensite suggests quenching by fast cooling but whether this quenching was intentional or not is a matter of debate particularly since there is no evidence for similar structure in any of the other objects analysed here. $\mathrm{Hv}_{(200)}=324$ (martensite), $\mathrm{Hv}_{(200)}=170$ (pearlite). Large number of slag inclusions traverse, predominantly, the low carbon area.

\section{c. Miscellaneaous}

879. Arrowhead. 9 th -8 th c BC. Length: $3 \mathrm{~cm}$. Width (max): $1.8 \mathrm{~cm}$. Metallographic section revealed no remaining metal, due to its minimal thickness.

854. Spit. 9th-8th c BC. Length: $7.1 \mathrm{~cm}$. Width: $.5 \mathrm{~cm}$. Metallographic section was cut $2.5 \mathrm{~cm}$ from one end.

Fine equiaxial ferrite. One corner of the section displays some degree of carburisation with evidence of widmanstatten structure but this is secondary and non 


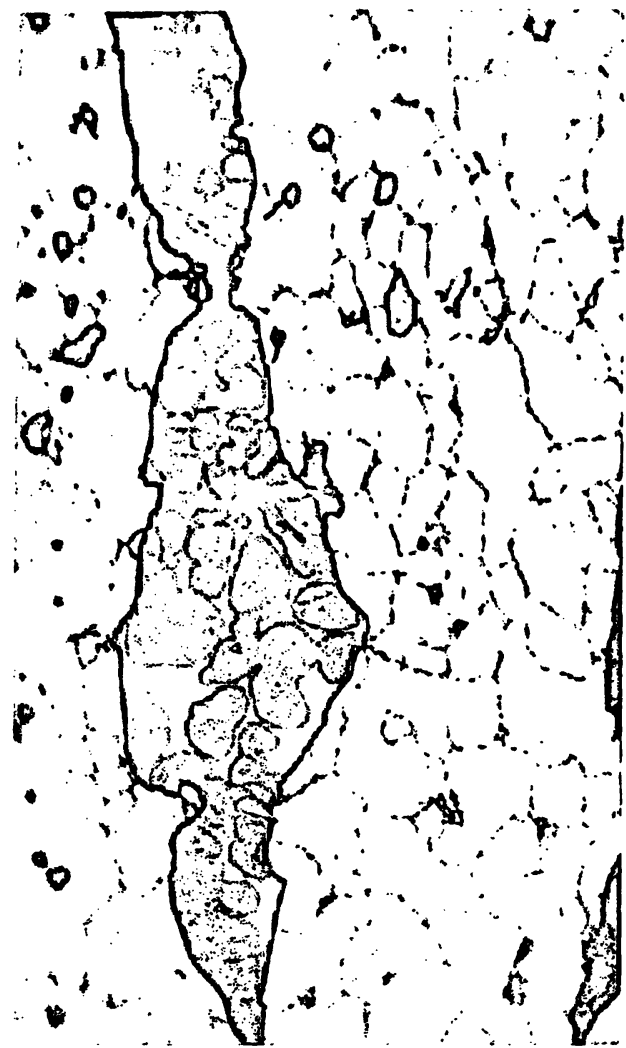

Fig. 51. Knife Inv. Nr. 988. Two phase slag inclusion in ferritic iron. Glassy matrix (dark gray), iron oxide dendrides (light gray). Scale 400:1

intentional. Slag inclusions are many, scattered throughout the section. No particular effort seems to have gone into removing them since the object could not have been considered functional. $\mathrm{Hv}_{(100)}=110$.

\section{Chemical Analysis}

Chemical analysis was carried out with the microprobe to establish the presence of impurities in the metal and the composition of slag inclusions (Fig. 51). The metal revealed absence of phosphorus or sulfur or other metallic elements apart from occasional traces of copper. The presence of traces of copper in the metal is not uncharacteristic and suggests that copper oxide, probably an accidental addition, made its way into the smelting furnacc. Slag inclusions can originate either from the smelting cycle - they were never completely removed during the consolidation of the bloom - or during the smithing or in the forge-welding of two pieces of iron. In the latter, joining can be achieved with the addition of sand $\left(\mathrm{SiO}_{2}\right)$ which reacts with the oxidised surface of the metal $(\mathrm{FeO})$ to form a slag $\left(2 \mathrm{FeO} . \mathrm{SiO}_{2}\right)$.

Table 1 presents the chemical analysis of the two common phases in slag inclusions (Fig. 51), namely iron oxide dendrites (wustite) and a glassy silicate matrix. The composition of these particular phases suggests that they could have arisen from either the smelting or the smithing stage. More important, they do not contain any characteristic trace elements which could direct the investigation to a particular type of ore body. They suggest a hematite/limonite type of ore, one that is widely available at any geographical region. Thus, at this stage of the investigation, it is rather difficult to establish whether the raw metal for the Vergina artefacts was produced locally or was imported.

\section{Discussion}

There exist very few published analyses of greek iron artefacts in relation to the plethora of iron excavated in sites post-dating the Mycenean period in Greece (Richardson 1934; Livadefs 1956; Varoufakis 1973; Conofagos and Papadimitriou 1981). Presently, this trend is beginning to reverse and a number of metallographic and chemical analyses of iron objects are currently underway (Photos 1987).

For a period of approximately twenty five centuries (10th c BC to 15th c AD), iron (wrought iron with less than $.05 \% \mathrm{C}$ ) was produced in Europe in small shafted furnaces (bloomeries) via the solid state reduction of iron ore in the presence of a reducing medium like charcoal. That meant that the iron (bloom) was at no stage molten (as is the case in the modern blast furnace) but only the slag was, id est the waste non-metalliferous material which separated from the metal by running to the bottom of the furnace.

As a result of the solid state reduction, the carbon content of the bloom far from being uniform was variable depending on the localised conditions of the furnace. Thus, the smith had to remedy the situation by choosing the right sections according to the type of object he intended to make as well as follow the correct heat

Table 1. Chemical composition of two phases in slag inclusions

\begin{tabular}{|c|c|c|c|c|c|c|c|c|c|}
\hline phase & $\mathrm{MgO}$ & $\mathrm{Al}_{2} \mathrm{O}_{3}$ & $\mathrm{SiO}_{2}$ & $\mathrm{P}_{2} \mathrm{O}_{5}$ & $\mathrm{~K}_{2} \mathrm{O}$ & $\mathrm{CaO}$ & $\mathrm{TiO}_{2}$ & $\mathrm{MnO}$ & $\mathrm{FeO}$ \\
\hline wustite & 0.38 & 0.30 & 0.36 & 0.12 & 0.05 & 0.00 & 0.00 & 0.78 & 97.04 \\
\hline silicate matrix & 1.00 & 8.08 & 41.87 & 0.37 & 3.96 & 16.99 & 0.18 & 1.26 & 24.37 \\
\hline
\end{tabular}


treatment to achieve the desired properties. In addition he would bave to remove the remains of trapped slag which in the long run would act as corrosion nuclei while in the short run would be weak points in the metal fabric given their glassy nature ${ }^{237}$.

Steel, an alloy of iron and carbon much harder than iron, contains carbon ranging between .1 and $1.8 \% \mathrm{C}$. Hardness is enhanced if the alloy is cooled quickly from a high temperature in a medium like water or oil. Thus, for steel production both the right carbon content had to be present and the correct heat treatment had to be followed.

Some of the observations presented above in reference to the structure and properties of the iron-carbon alloy seem to have been suprisingly clear to the smiths of the Vergina objects as early as the 9th c BC. The choice of the raw material seemed to have been based on the type of object to be manufactured. Thus, for parts which were not clearly functional like the hilt of a sword (no. 853) and spits (no. 854) sections of bloom characteristic for their low carbon content (ferrite) were chosen. In addition, no effort was made to remove the large number of slag inclusions.

On the other hand, the blades of knives and swords were all chosen for their higher carbon content, although the amount barely surpassed that for mild steel $(.5 \% \mathrm{C})$. Often a gradient in carburisation, in decreasing order from the cutting edge to the dull edge, was evident (nos. 833, 854). The preferential carburisation of the cutting edge coupled with uniform grain distribution (nos. 853, 988) throughout the blade also point to a very adequate control of the reducing environment in the forge particularly when some objects seem to have been exposed to prolonged heating periods (spheroidisation in no. 988). In only one sample is there possible evidence for decarburisation (no. 823) in the hearth, the result of local oxidising conditions.

Controlled carburisation is one step towards making functional, good quality steel. The other is enhancing the hardness by the correct heat treatment. In only one occasion (no. 854) is there evidence for possible quenching of the Vergina artefacts (presence of martensite). The cutting edge of this knife-seems to have been cooled with care. It is clearly not a case of the smith dipping the object in water just so that he can handle it. Nevertheless,

237 These general observations and a host of other more detailed ones have arisen through the metallographic examination of ancient iron artefacts from various geographical regions in Europe and Asia and the experimental work on bloomery smelting of a variety of iron ores carried out by a number of investigators (Tylecote et al. 1971; Cleere 1972; Tylecote and Gilmour 1986; Tylecote 1987, to mention only a select few). the fact that martensite was absent in all other samples may suggest that hardening via quenching was not yet well understood. However, it is possible that examination of additional artefacts may disprove this preliminary observation.

At his stage of the investigation it is difficult to establish, on chemical grounds, whether the objects were produced locally or were imported. The mineralogy of the slag inclusions do not offer any clues as to a characteristic type of iron deposit other than the widely available limonite/hematite, while the presence of manganese in the slag and some traces of copper in the metal do not give many leads. Furthermore, the absence of slag samples and the presently inavailable information about local ore deposits do not make the task easier. The question will undoubtedly be elucidated in the future with the examination of additional artefacts, analyses of ores and the study of comparative material from other Macedonian cemeteries and settlement sites.

\section{References}

Cleere 1972: H. Cleere, Iron making in a Roman furnace. Britannia 2, 1972, 203-217.

Conofagos and Papadimitriou 1981: C.Conofagos and G. Papadimitriou, Les crampons en acier de l'Erechtheion ont été fabriqués selon la technique qu'on retrouve au Moyen Age pour les «Epées Damassées». Proc. of the Academy of Athens 56, 1981, 173-190. Greek with French summary.

Livadefs 1956: S. Livadefs, The structural iron of the Parthenon. Journal of the Iron and Steel Institute 182, 1956, 49-66.

Photos 1987: E. Photos, Early Extractive Iron Metallurgy in N Greece; a unified approach to regional archaeometallurgy. Unpublished PhD thesis. University of London (1987).

Richardson 1934: H.G. Richardson, Iron prehistoric and ancient. American Journal of Archaeology 38, 1934, $555-583$.

Tylecote et al. 1971: R.F. Tylecote, J.N. Austin and A.E. Wraith, Mechanism of bloomery process in shaft furnaces. Journal of the Iron and Steel Institute 209, 1971, 342-362.

Tylecote and Gilmour 1986: R.F. Tylecote and B.J.J. Gilmour, The Metallography of Early Ferrous Edge Tools and Edged Weapons. British Archaeological Reports, British Series 155 (1986).

Tylecote 1987: R.F. Tylecote, The Early History of Metallurgy in Europe. Longman Archaeology Series (London 1987).

Varoufakis 1973: G. Varoufakis, Investigation into three iron spearheads of the 7th and 6th c BC. Metalleiologika ke Metallourgika Chronika 10, 1973, 23-34 (in Greek). 


\title{
Anhang 2
}

\section{Anthropologische Untersuchung der menschlichen Zahnfunde}

\author{
von Ursula Wittwer-Backofen, Mainz"
}

Malamias, Grabhügcl B, Grab II

Vorhanden ist 1 menschliche Zahnkrone, lediglich Zahnschmelz, abcr kcin Dentin (Zahnbcin) erhalten:

(nach FDI) 35 (2. Milchmolar Unterkiefer links)

ALTER: Milchzahn mit leichten Abrasionsspuren, ca. 3-5 Jahre.

GESCHLECHT: bei Milchgebiß nicht feststellbar. BESONDERHEITEN: keine.

Malamas, Grabhügel B, Grab VI

Vorhanden sind 6 menschliche Zahnkronen, lediglich Zahnschmelz, aber kein Dentin erhalten, im einzelnen:
18 (3. Molar Oberk. re.)
16 (1. Molar Oberk. re.)
25 (2. Prämolar Oberk. li.)
27 (2. Molar Oberk. li.)
28 (3. Molar Oberk. li.)
35 (2. Prämolar Unterk. li.)

ZAHNSTATUS:

\begin{tabular}{l|l}
$18-16-----$ & $----25-2728$ \\
\hline--------1 & $----35---$
\end{tabular}

Alle diese Zähne gehören zu einem Individuum.

GESCHLECHT: aufgrund der mesio-distalen und bucco-lingualen Maße der Zähne möglicherweise eher weiblich.

ALTER: aufgrund der Zahnabrasion (nach SCOTT) ca. 12-20 Jahre.

BESONDERHEITEN: keine Karies, kein Zahnstein.

Malamas, Grabhügel $\Gamma$, Grab I

Vorhanden sind 17 menschliche Zahnkronen, lediglich Zahnschmelz, aber kein Dentin erhalten, im einzelnen:

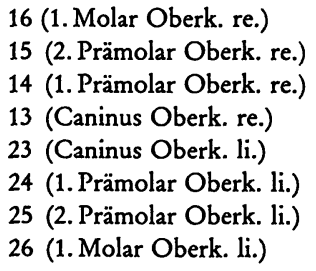

* Frau U. Wittwer-Backofen war freundlicherweise bereit, die Untersuchung der Zahnfunde aus der Rettungsgrabung von 1970 zu übernehmen. Es war nicht möglich, die Zahnfunde aus den Grabungen Andronikos und Petsas miteinzubeziehen oder Vergleichsmaterial gleicher Zeitstellung aus Mazedonien auszuwerten, um so das isolierte Zahnmaterial aus den vier Gräbern in statistisch abgesicherte Meßwerte einzuordnen. (K.Rh. u. I. K.-D.)

\author{
27 (2. Molar Oberk. li.) \\ 36 (1. Molar Unterk. li.) \\ 37 (2. Molar Unterk. li.) \\ 43 (Caninus Unterk. re.) \\ 44 (1. Prämolar Unterk. re.) \\ 45 (2. Prämolar Unterk. re.) \\ 47 (2. Molar Unterk. re.)
}

\section{ZAHNSTATUS:}

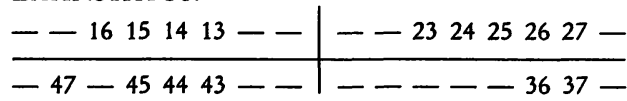

Alle diese Zähne gehören zu einem Individuum.

GESCHLECHT: aufgrund der mesio-distalen und bucco-lingualen Maße der Zähne (nach DITCH und ROSE) möglicherweise eher weiblich.

ALTER: aufgrund der Zahnabrasion (nach SCOTT) ca. 20-40 Jahre.

BESONDERHEITEN: keine Karies, kein Zahnstein.

Jedoch zwei Zähne eines zweiten Individuums im Fundkomplex:

$$
17 \text { (2. Molar Oberk. re.) }
$$

GESCHLECHT: aufgrund der Zahnmaße (siehe oben) möglicherweise eher weiblich.

ALTER: aufgrund der Zahnabrasion max. $30 \mathrm{Jahre}$, mindestens aber $18 \mathrm{Jahre}$.

Malamas, Grabhügel $\Gamma$, Grab II

Vorhanden sind 19 menschliche Zahnkronen, lediglich Zahnschmelz, aber kein Dentin erhalten, im einzelnen:
18 (3. Molar Oberk. re.)
17 (2. Molar Oberk. re.)
16 (1. Molar Oberk. re.)
15 (2. Prämolar Oberk. re.)
25 (2. Prämolar Oberk. li.)
26 (1. Molar Oberk. li.)
27 (2. Molar Oberk. li.)
28 (3. Molar Oberk. li.)
33 (Caninus Unterk. li.)
34 (1. Prämolar Unterk. li.)
35 (2. Prämolar Unterk. li.)
36 (1. Molar Unterk. li.)
37 (2. Molar Unterk. li.)
38 (3. Molar Unterk. li.)
45 (2. Prämolar Unterk. re.)
46 (1. Molar Unterk. re.)
47 (2. Molar Unterk. re.)
48 (3. Molar Unterk. re.) 
ZAHNSTATUS:

18 $171615----\mid----25262728$

\begin{tabular}{l}
$48474645-\ldots-\ldots$ \\
\hline-333435363738
\end{tabular}

Diese 18 Zähne gehören zu einem Individuum.

GESCHLECHT: aufgrund der mesio-distalen und bucco-lingualen $\mathrm{Maße}$ der Zähne möglicherweise eher männlich.

ALTER: aufgrund der Zahnabrasion ca. 20-40 Jahre. BESONDERHÉITEN: keine Karies, kein Zahnstein, aber leichte Zahnschmelzhypoplasien (Fehlausbildung des Zahnschmelzes als Folge von Mangelernährung während des Zahnbildungsprozesses in der Kindheit).

Ein Zahn eines weiteren Individuums vorhanden: 46 (1. Molar Unterk. re.)

wenig abgekaut, möglicherweise noch subadult (unter 20 Jahre), Geschlecht unklar.

Ursula Wittwer-Backofen, Institut für Antbropologie, Universität, Saarstr. 21, D6500 Mainz 